REVIEWS OF MODERN PHYSICS, VOLUME 90, JULY-SEPTEMBER 2018

\title{
Quantum-enhanced measurements without entanglement
}

\author{
Daniel Braun* \\ Institute of Theoretical Physics, University Tübingen, Auf der Morgenstelle 14, \\ 72076 Tübingen, Germany
}

\author{
Gerardo Adesso \\ Centre for the Mathematics and Theoretical Physics of Quantum Non-Equilibrium Systems \\ (CQNE), School of Mathematical Sciences, University of Nottingham, University Park, \\ Nottingham NG7 2RD, United Kingdom
}

\author{
Fabio Benatti \\ Department of Physics, University of Trieste, 34151 Trieste, Italy \\ and Istituto Nazionale di Fisica Nucleare, Sezione di Trieste, 34151 Trieste, Italy \\ Roberto Floreanini \\ Istituto Nazionale di Fisica Nucleare, Sezione di Trieste, 34151 Trieste, Italy
}

\author{
Ugo Marzolino \\ Department of Physics, Faculty of Mathematics and Physics, \\ University of Ljubljana, 1000 Ljubljana, Slovenia \\ and Division of Theoretical Physics, Ruđer Bošković Institute, 10000 Zagreb, Croatia
}

Morgan W. Mitchell

ICFO_Institut de Ciencies Fotoniques, The Barcelona Institute of Science and Technology, 08860 Castelldefels, Barcelona, Spain and ICREA-Institució Catalana de Recerca i Estudis Avançats, 08015 Barcelona, Spain

\section{Stefano Pirandola \\ Computer Science and York Centre for Quantum Technologies, University of York, York YO10 5GH, United Kingdom}

(published 5 September 2018)

\begin{abstract}
Quantum-enhanced measurements exploit quantum mechanical effects for increasing the sensitivity of measurements of certain physical parameters and have great potential for both fundamental science and concrete applications. Most of the research has so far focused on using highly entangled states, which are, however, difficult to produce and to stabilize for a large number of constituents. In the following alternative mechanisms are reviewed, notably the use of more general quantum correlations such as quantum discord, identical particles, or nontrivial Hamiltonians; the estimation of thermodynamical parameters or parameters characterizing nonequilibrium states; and the use of quantum phase transitions. Both theoretically achievable enhancements and enhanced sensitivities not primarily based on entanglement that have already been demonstrated experimentally and indicate some possible future research directions are described.
\end{abstract}

DOI: 10.1103/RevModPhys.90.035006

\section{CONTENTS}

I. Introduction

A. Aim and scope

B. Parameter estimation theory

C. Quantum parameter estimation theory

II. Quantum Correlations Beyond Entanglement

*daniel.braun@uni-tuebingen.de
A. Parallel versus sequential strategies in unitary quantum metrology

B. General results on the usefulness of entanglement

C. Role of quantum discord in parameter estimation with mixed probes

D. Black-box metrology and the interferometric power

E. Quantum estimation of bosonic loss

F. Gaussian quantum metrology

G. Quantum channel discrimination

H. Average precision in black-box settings 
III. Identical Particles

A. Particle and mode entanglement

1. Particle entanglement

2. Mode entanglement

B. Mode entanglement and metrology: Bosons

C. Mode entanglement and metrology: Fermions

IV. More General Hamiltonians

A. Nonlinear Hamiltonians

B. Proposed experimental realizations

1. Nonlinear optics

2. Ultracold atoms

3. Nanomechanical oscillators

4. Nonlinear Faraday rotation

C. Observations and commentary

D. Nonlinear measurement under number-optimized conditions

E. Signal amplification with nonlinear Hamiltonians

F. Other modifications of the Hamiltonian

1. Lifting spectral limitations

2. Decoherence-enhanced measurements

3. Coherent averaging

4. Quantum feedback schemes

V. Thermodynamical and Nonequilibrium Steady States

A. Thermodynamical states and thermal phase transitions

1. Role of quantum statistics

2. Interferometric thermometry

B. Thermodynamical states and quantum phase transitions

1. Quasifree fermion models

2. Hubbard models

3. Spin-1/2 systems

4. Topological quantum phase transitions

C. Nonequilibrium steady states

D. Adaptive measurements

VI. Outlook

Acknowledgments

References

\section{INTRODUCTION}

\section{A. Aim and scope}

Quantum-enhanced measurements aim at improving measurements of physical parameters by using quantum effects. The improvement sought is an enhanced sensitivity for a given amount of resources such as mean or maximum energy used, number of probes, number of measurements, and integration time. Ideas in this direction go back at least to the late 1960 s when the effect of quantum noise on the estimation of classical parameters started to be studied in a systematic way using appropriate mathematical tools (Helstrom, 1969; Holevo, 1982). In the early 1980s the first detailed proposals appeared on how to enhance the sensitivity of gravitational wave detectors by using squeezed light (Caves, 1980, 1981). Nowadays, squeezed light is routinely produced in many labs and used for instance to enhance sensitivity in gravitational wave observatories (Aasi et al., 2013; Chua, 2015).

Quantum-enhanced measurements have the potential of enabling many important applications, both scientific and technological. Besides gravitational wave detection, there are proposals or demonstrations for the improvement of time or frequency standards, navigation, remote sensing, measurement of very small magnetic fields (with applications to medical brain and heart imaging), measurement of the parameters of spacetime, thermometry, and many more. The literature on the topic of quantum metrology is vast and for a general introduction available reviews are Giovannetti, Lloyd, and Maccone (2006), Paris (2009), Wiseman and Milburn (2009), Pezzè and Smerzi (2014), Tóth and Apellaniz (2014), Degen, Reinhard, and Cappellaro (2016), and Pezzè et al. (2016).

From the theoretical side, the standard tool for evaluating a possible quantum enhancement has become the so-called quantum Cramér-Rao bound (Helstrom, 1969; Holevo, 1982; Braunstein and Caves, 1994; Braunstein, Caves, and Milburn, 1996). It provides a lower bound on the variance $\operatorname{Var}\left(\theta_{\text {est }}\right)$ of any unbiased estimator function $\theta_{\text {est }}$ that maps observed data obtained from arbitrary quantum measurements to an estimate of the parameter $\theta$. The bound is optimized over all possible measurements and data analysis schemes in a sense made precise in Sec. I.B. In the limit of an infinite number of measurements the bound can be saturated. It thus represents a valuable benchmark that can in principle be achieved once all technical noise problems have been solved, such that only the unavoidable noise inherent in the quantum state itself remains.

A standard classical method of noise reduction is to average measurement results from $N$ independent, identically prepared systems. In a quantum mechanical formulation with pure states, the situation corresponds to having the $N$ quantum systems in an initial product state $|\psi\rangle=\otimes_{i=1}^{N}|\phi\rangle_{i}$. Suppose that the parameter is encoded in the state through a unitary evolution with a Hamiltonian $H(\theta)=\theta \sum_{i=1}^{N} h_{i}$, i.e., $|\psi(\theta)\rangle=\exp [-i H(\theta)]|\psi\rangle$. Based on the quantum CramérRao bound one can show that with $M$ final measurements the smallest achievable variance $\operatorname{Var}\left(\theta_{\text {est }}\right)$ of the estimation of $\theta$ is

$$
\operatorname{Var}\left(\theta_{\text {est }}\right)_{\min }=\frac{1}{N M(\Lambda-\lambda)^{2}},
$$

where $\Lambda$ and $\lambda$ are the largest and smallest eigenvalues of $h_{i}$, respectively, taken for simplicity here as identical for all subsystems (Giovannetti, Lloyd, and Maccone, 2006). In fact, this $1 / \sqrt{N}$ scaling can be easily understood as a consequence of the central limit theorem in the simplest case that one measures the systems independently. But since Eq. (1) is optimized over all measurements of the full system, it also implies that entangling measurements of all systems after the parameter has been encoded in the state cannot improve the $1 / \sqrt{N}$ scaling.

Unfortunately, there is no unique definition of the standard quantum limit (SQL) in the literature. Whereas in the described $1 / \sqrt{N}$ scaling $N$ refers to the number of distinguishable subsystems, the term standard quantum limit is used, for example, in quantum optics typically for a scaling as $1 / \sqrt{\bar{n}}$ with the average number of photons $\bar{n}$, which in the same mode are to be considered as indistinguishable (see Sec. III). In this context, the $1 / \sqrt{\bar{n}}$ scaling is also called the "shot-noise limit," referring to the quantum noise that arises from the fact that the electromagnetic energy is quantized in units of photons. Furthermore, the prefactor in these scaling behaviors is not fixed. Quite generally, the standard quantum limit may 
be defined as the best scaling that can be achieved when employing only "classical" resources.

While this is not yet a mathematical definition either, it becomes precise once the classical resources are specified in the problem at hand. This may be achieved adopting a resource-theory framework, in which classical states of some specific sort are identified and formalized as "free" states (i.e., given at no cost), and any other state is seen as possessing a resource content which may allow us to outperform free states in practical applications, leading specifically to quantumenhanced measurements beyond the standard quantum limit scaling. For instance, separable states are the free (classical) states in the resource theory of entanglement (Horodecki et al., 2009), while states diagonal in a reference basis are the free (classical) states in the resource theory of quantum coherence (Streltsov, Adesso, and Plenio, 2017). In quantum optics, Glauber's coherent states and their mixtures are regarded as the free (classical) states (Mandel and Wolf, 1965), and any other state can yield a nonclassical scaling. In the latter example, considering the mean photon number $\bar{n}$ as an additional resource, one can fix the prefactor of the standard quantum limit scaling, so that quantum enhancements are possible not only by improving the scaling law, but also by changing the prefactor.

However, basing our review exclusively on a resourcetheory picture would be too restrictive as cases of enhanced sensitivity are readily available for which no resource theory has been worked out yet [see Brandão and Gour (2015) and references therein for a recent overview of existing resource theories]. Examples are the use of quantum phase transitions for which one can compare the sensitivity at the phase transition with the sensitivities away from the phase transition, or instances of Hamiltonian engineering, for which one can evaluate the effect of added terms in the Hamiltonian. Rather than developing resource theories for all these examples, which would be beyond the scope of this review, the enhancements achievable compared to the sensitivity without the use of the mechanism under consideration will be pointed out.

Based again on the quantum Cramér-Rao bound one can show that initially entangled states can improve the scaling to $1 / N$ (Giovannetti, Lloyd, and Maccone, 2006), known as the "Heisenberg limit." Similar to the standard quantum limit, there is no unique definition of the Heisenberg limit in the literature (see the remarks in Sec. IV.A). Nevertheless, achieving the Heisenberg limit has been the goal of large experimental and theoretical efforts over the last two decades. However, only few experiments achieved the $1 / N$ scaling of the Heisenberg limit and only for very small numbers of subsystems, where the scaling advantage is still far from allowing one to beat the best possible classical measurements. This has several reasons: First of all, it is already very difficult to achieve even the standard quantum limit, as all nonintrinsic noise sources have to be eliminated. Second, resources such as photons are cheap, such that classically one can operate with very large photon numbers, whereas entangled states with large photon numbers are difficult to produce. Third, and most fundamentally, quantum-enhanced measurement schemes are plagued by decoherence. Indeed, it has been shown that a small amount of Markovian decoherence brings the $1 / N$ scaling for certain highly entangled states back to the $1 / \sqrt{N}$ scaling of the standard quantum limit (Huelga et al., 1997; Kołodyński and Demkowicz-Dobrzański, 2010; Escher, de Matos Filho, and Davidovich, 2011). The reduction to the standard quantum limit also affects the estimation of noise in programmable and teleportation-covariant channels (Laurenza et al., 2018). Recent research has focused on finding optimal states in the presence of decoherence, and at least for nonMarkovian noise, a certain improvement can still be obtained from entangled states (Matsuzaki, Benjamin, and Fitzsimons, 2011; Chin, Huelga, and Plenio, 2012). Also niche applications are possible, for which the light intensity must be very small, as in some biological applications. Nevertheless, it appears worthwhile to think about alternative possible quantum enhancement principles other than the use of highly entangled states, and this is the focus of the present survey.

Many results have been obtained over the past years for such alternative schemes that are worth a comprehensive and exhaustive review that compares their usefulness with respect to the main-stream research focused on highly entangled states. The review is structured by different ways of breaking the conditions that are known to lead to standard quantum limit scaling of the sensitivity. First, by going away from pure states, more general forms of quantum correlations such as quantum discord become possible. These become naturally important for estimation of loss parameters, quantum illumination problems, and other applications that typically involve the loss of probes. Second, in the derivation of the standard quantum limit the quantum systems are distinguished by an index $i$, which supposes that they are distinguishable. Cold atoms, on the other hand, have to be considered in general as indistinguishable particles, and the same is true for photons, which have been used for quantum-enhanced measurements from the very beginning. Hence, statements about the necessity of entanglement have to be reexamined for indistinguishable particles. It turns out that the permutational symmetry of the quantum states required due to indistinguishability of the particles leads immediately to the level of quantum-enhanced sensitivity that for distinguishable particles would require to entangle them. Third, the structure of the Hamiltonian is rather restrictive: (a) Many Hamiltonians do not have a bound spectrum characterized by the largest and smallest eigenvalues $\Lambda$ and $\lambda$ as assumed in Eq. (1). Indeed, one of the most common systems used in quantum metrology, the harmonic oscillator that represents, e.g., a single mode of an electromagnetic field, has an unbound spectrum. And (b), the Hamiltonian $H(\theta)=\theta \sum_{i=1}^{N} h_{i}$ does not allow for any interactions. Taking into account these freedoms opens the path to many new forms of enhanced sensitivity. Fourth, unitary evolutions with a Hamiltonian that depends on the parameter are not the only way of coding a parameter in a state. In statistical mechanics, for example, there are parameters that describe the statistical ensemble, such as temperature or chemical potential for systems in thermal equilibrium, but which are not of Hamiltonian origin. The same is true for nonequilibrium states. For many of these situations, the corresponding quantum Cramér-Rao bounds have been obtained only recently, and it often turned out that improvements beyond the standard quantum limit should be possible. 
Furthermore, it is known even in classical statistical physics that phase transitions can lead to diverging susceptibilities and hence greatly enhanced sensitivities. The same is true for quantum phase transitions, whose use for quantum-enhanced measurements will be reviewed.

While a growing number of researchers are investigating possibilities of breaking the standard quantum limit without using entanglement (Tilma et al., 2010), these still appear to be a minority. The situation is comparable to other aspects and fields of quantum information treatment, where previously it was thought that entanglement is necessary. For example, for a long time entanglement has been considered as necessary for nonlocality, until it was realized that certain aspects of nonlocality can arise without entanglement (Bennett et al., 1999). Recent reviews of quantum-enhanced measurement schemes using entanglement (Paris, 2009; Giovannetti, Lloyd, and Maccone, 2011; Pezzè and Smerzi, 2014; Tóth and Apellaniz, 2014; Degen, Reinhard, and Cappellaro, 2016; Pezzè et al., 2016) are available and the review does not survey this vast literature here, but focus rather exclusively on quantum-enhanced measurement schemes that are not essentially based on the use of entanglement, hoping that our review will stimulate research in these directions. Before reviewing these schemes, a short introduction to parameter estimation theory and the precise definition of the quantum Cramér-Rao bound are given. A more elaborate pedagogical introduction to classical and quantum parameter estimation theory can be found in Fraïsse (2017).

\section{B. Parameter estimation theory}

Consider the following task in classical statistical analysis: Given a probability distribution $p_{\theta}(x)$ of a random variable $x$ that continuously varies as a function of a single real parameter $\theta$, estimate $\theta$ as precisely as possible from $M$ samples drawn, i.e., a set of random values $\left\{x_{i}\right\}, i=1,2, \ldots, M$. This $M$ sample is denoted as $\boldsymbol{x}$ for short and denote the probability to find the drawn samples in the intervals $x_{i} \cdots x_{i}+d x_{i}$ as $p_{\theta}(\boldsymbol{x}) d^{M} \boldsymbol{x}$, with $d^{M} \boldsymbol{x}=d x_{1} \cdots d x_{M}$. For independently drawn, identically distributed samples, $p_{\theta}(\boldsymbol{x})=p_{\theta}\left(x_{1}\right) \times \cdots \times p_{\theta}\left(x_{M}\right)$, but the formalism allows for arbitrary joint-probability distributions $p_{\theta}(\boldsymbol{x})$ i.e., also correlations between different samplings of the distribution. For simplicity, the support of $x$ is taken to be the real numbers.

The task is accomplished by using an estimator function $\theta_{\text {est }}\left(x_{1}, \ldots, x_{M}\right)$ that takes as input the drawn random values and nothing else and outputs an estimate of the parameter $\theta$. Many different estimator functions are possible, some more useful than others. Through its random arguments the estimator will itself fluctuate from one sample to another. First, one wants to have an estimator that on average gives the true value of $\theta, E\left(\theta_{\text {est }}\right)=\theta$, where $E(\cdots)=\int d^{M} \boldsymbol{x} p_{\theta}(\boldsymbol{x})(\cdots)$ is the mean value of a quantity over the distribution. This should hold at least in an infinitesimal interval about the true value of $\theta$; such an estimator is called "unbiased." Second, one wants the estimator to fluctuate as little as possible. The latter request makes sense only together with the first one, as a constant estimator of course would not reproduce the correct value of $\theta$ in most cases. Now consider the following chain of equalities, valid for an unbiased estimator:

$$
\begin{aligned}
1 & =\frac{\partial}{\partial \theta} E\left(\theta_{\mathrm{est}}\right)=\int d^{M} \boldsymbol{x} \frac{\partial}{\partial \theta} p_{\theta}(\boldsymbol{x}) \theta_{\mathrm{est}}(\boldsymbol{x}) \\
& =\int d^{M} \boldsymbol{x} p_{\theta}(\boldsymbol{x})\left(\frac{\partial}{\partial \theta} \ln p_{\theta}(\boldsymbol{x})\right) \theta_{\mathrm{est}}(\boldsymbol{x}) \\
& =\int d^{M} \boldsymbol{x} p_{\theta}(\boldsymbol{x})\left(\frac{\partial}{\partial \theta} \ln p_{\theta}(\boldsymbol{x})\right)\left[\theta_{\mathrm{est}}(\boldsymbol{x})-\theta\right] \\
& =\left\langle\frac{\partial \ln p_{\theta}}{\partial \theta}, \theta_{\mathrm{est}}-\theta\right\rangle
\end{aligned}
$$

The step before the last one holds because $\theta\left(\partial / \partial_{\theta}\right) E(1)=0$ due to the normalization of the probability distribution valid for all values of $\theta$. The scalar product in the last step is defined for any two real functions $a(\boldsymbol{x})$ and $b(\boldsymbol{x})$ as $\langle a, b\rangle=$ $\int d^{M} \boldsymbol{x} p_{\theta}(\boldsymbol{x}) a(\boldsymbol{x}) b(\boldsymbol{x})$. The Cauchy-Schwarz inequality for this scalar product immediately implies the classical Cramér-Rao (lower) bound for the variance of the estimator

$$
\operatorname{Var}\left(\theta_{\mathrm{est}}\right) \geq \frac{1}{J_{\theta}^{(M)}}
$$

where the classical Fisher information $J_{\theta}$ is defined as

$$
\begin{aligned}
J_{\theta}^{(M)} & =\int d^{M} \boldsymbol{x} p_{\theta}(\boldsymbol{x})\left(\frac{\partial \ln p_{\theta}(\boldsymbol{x})}{\partial \theta}\right)^{2} \\
& =\int d^{M} \boldsymbol{x} \frac{1}{p_{\theta}(\boldsymbol{x})}\left(\frac{\partial p_{\theta}(\boldsymbol{x})}{\partial \theta}\right)^{2} .
\end{aligned}
$$

The bound can be saturated iff the two vectors in the scalar product are parallel, i.e., for $\partial \ln p_{\theta}(\boldsymbol{x}) / \partial \theta=$ $A(\theta)\left[\theta_{\text {est }}(\boldsymbol{x})-\theta\right]$, where $A(\theta)$ is a possibly $\theta$-dependent proportionality factor. If one differentiates this condition once more and then integrates it over with $p_{\theta}(\boldsymbol{x})$, one finds that $A(\theta)=J_{\theta}^{(M)}$. Hence, an unbiased estimator exists iff there is a function $f(\boldsymbol{x})$ independent of $\theta$ such that $\partial \ln p_{\theta} / \partial \theta=$ $J_{\theta}^{(M)}[f(\boldsymbol{x})-\theta]$. In that case one can choose $\theta_{\text {est }}(\boldsymbol{x})=f(\boldsymbol{x})$. One can show that for many (but not all) members of the family of exponential probability distributions, i.e., distributions that can be written in the form $p_{\theta}(x)=$ $a(x) \exp [b(\theta) c(x)+d(\theta)]$ with some functions $a(x), b(\theta)$, $c(x)$, and $d(\theta)$, this condition is satisfied, meaning that in such cases the Cramér-Rao bound can be saturated even for finite $M$. For $M \rightarrow \infty$ and identically, independently distributed samples, the so-called maximum-likelihood estimator saturates the bound. One easily shows from Eq. (4) that the Fisher information is additive, such that for independently drawn, identically distributed samples $J_{\theta}^{(M)}=M J_{\theta}$ with $J_{\theta} \equiv J_{\theta}^{(1)}$.

\section{Quantum parameter estimation theory}

In quantum mechanics the state of a system is given by a density matrix $\rho_{\theta}$, i.e., a positive Hermitian operator with trace equal to 1 that can depend on the parameter $\theta$, which is assumed to be a classical parameter. Random data are created when measuring some observable of the system whose statistics will depend on $\theta$ through the quantum state $\rho_{\theta}$. Again, $\theta$ must be estimated as precisely as possible based on the measurement data (see Fig. 1). 


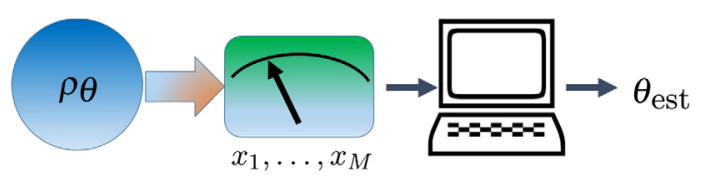

FIG. 1. General setup of quantum parameter estimation. A quantum measurement of a system in quantum state $\rho_{\theta}$ that depends on a classical parameter $\theta$ is performed (general POVM measurement) and produces data $x_{1}, \ldots, x_{M}$. The data are analyzed with an estimator function that outputs an estimate $\theta_{\mathrm{est}}$ of $\theta$. The goal is to obtain an unbiased estimate with as small as possible statistical fluctuations.

The most general measurements are so-called positiveoperator-valued measures (POVM). These are measurements that generalize and include projective von Neumann measurements and are relevant, in particular, when the system is measured through an ancilla system to which it is coupled (Peres, 1993). They consist of a set of positive operators $M_{x}$, where $x$ labels possible measurement outcomes and again $x \in \mathbb{R}$ for simplicity. They obey a completeness relation $\int_{\mathbb{R}} M_{x} d x=\mathbb{I}$, where $\mathbb{I}$ is the identity operator on the Hilbert space of the system. The probability density to find outcome $x$ is given by $p_{\theta}(x)=\operatorname{tr}\left(\rho_{\theta} M_{x}\right)$, and it is through this equation that the contact with the classical parameter estimation theory can be made: Plugging in $p_{\theta}(x)$ into Eq. (4) with $M=1$, the Fisher information is

$$
\begin{aligned}
J_{\theta} & =\int d x \frac{1}{\operatorname{tr}\left(\rho_{\theta} M_{x}\right)}\left[\operatorname{tr}\left(\frac{\partial \rho_{\theta}}{\partial \theta} M_{x}\right)\right]^{2} \\
& =\int d x \frac{1}{\operatorname{tr}\left(\rho_{\theta} M_{x}\right)}\left[\operatorname{tr}\left(\frac{1}{2}\left(\rho_{\theta} L_{\rho_{\theta}}+L_{\rho_{\theta}} \rho_{\theta}\right) M_{x}\right)\right]^{2},
\end{aligned}
$$

where in the last step $L_{\rho_{\theta}}$ is the so-called symmetric logarithmic derivative, defined indirectly through

$$
\frac{\partial \rho_{\theta}}{\partial_{\theta}}=\frac{1}{2}\left(\rho_{\theta} L_{\rho_{\theta}}+L_{\rho_{\theta}} \rho_{\theta}\right),
$$

in analogy to the classical logarithmic derivative $\partial \ln \rho_{\theta} / \partial_{\theta}$. Compared to the classical case, one has in the quantum mechanical setting the additional freedom to choose a suitable measurement in order to obtain a distribution $p_{\theta}(x)$ that contains as much information as possible on the parameter $\theta$. Based on Eq. (5), one can find a similar chain of inequalities as in the classical case based on the Cauchy-Schwarz inequality that leads to the bound

$$
J_{\theta} \leq I_{\theta} \equiv \operatorname{tr}\left(\rho_{\theta} L_{\rho_{\theta}}^{2}\right)
$$

where $I_{\theta}$ is known as the "quantum Fisher information."

Similarly as for the classical Fisher information, the quantum Fisher information of uncorrelated states is additive (Fujiwara and Hashizumé, 2002):

$$
I_{\theta}(\rho(\theta) \otimes \sigma(\theta))=I_{\theta}(\rho(\theta))+I_{\theta}(\sigma(\theta))
$$

such that for $M$ independent identical POVM measurements of the same system, prepared always in the same state, the total quantum Fisher information satisfies $I_{\theta}^{(M)}=M I_{\theta}$ with $I_{\theta} \equiv I_{\theta}^{(1)}$. Inequalities (3) and (7) then lead to the so-called quantum Cramér-Rao bound,

$$
\operatorname{Var}\left(\theta_{\mathrm{est}}\right) \geq \frac{1}{M I_{\theta}}
$$

Additivity of the quantum Fisher information also immediately implies the $1 / \sqrt{N}$ scaling in Eq. (1), as the quantum Fisher information of the $N$ uncorrelated subsystems is just $N$ times the quantum Fisher information of a single subsystem. Inequality (7) can be saturated with a POVM that consists of projectors onto eigenstates of $L_{\theta}$ (Helstrom, 1969; Holevo, 1982; Braunstein and Caves, 1994). As the quantum CramérRao bound is already optimized, no measurement of the whole system, even if entangling the individual systems, can improve the sensitivity when the parameter was already imprinted on a product state.

The quantum Cramér-Rao bound has become the most widely used quantity for establishing the ultimate sensitivity of measurement schemes. It derives its power from the facts that first it is already optimized over all possible data analysis schemes (unbiased estimator functions) and all possible (POVM) measurements, and that second it can be saturated at least in the limit of infinitely many measurements and using the optimal POVM consisting of projectors onto the eigenstates of $L_{\rho_{\theta}}$.

Braunstein and Caves (1994) showed that $I_{\theta}$ is a geometric measure on how much $\rho(\theta)$ and $\rho(\theta+d \theta)$ differ, where $d \theta$ is an infinitesimal increment of $\theta$. The geometric measure is given by the Bures distance,

$$
d s_{\text {Bures }}^{2}(\rho, \sigma) \equiv 2[1-\sqrt{F(\rho, \sigma)}]
$$

where the fidelity $F(\rho, \sigma)$ is defined as

$$
F(\rho, \sigma)=\left\|\rho^{1 / 2} \sigma^{1 / 2}\right\|_{1}^{2},
$$

and $\|A\|_{1} \equiv \operatorname{tr} \sqrt{A A^{\dagger}}$ denotes the trace norm (Miszczak et al., 2009). With this Braunstein and Caves (1994) showed that

$$
I_{\theta}=4 d s_{\text {Bures }}^{2}(\rho(\theta), \rho(\theta+d \theta)) / d \theta^{2},
$$

unless the rank of $\rho(\theta)$ changes with $\theta$ and thus produces removable singularities (Banchi, Braunstein, and Pirandola, 2015; Šafránek, 2017), a situation not considered in this review. The quantum Cramér-Rao bound thus offers the physically intuitive picture that the parameter $\theta$ can be measured the more precisely the more strongly the state $\rho(\theta)$ depends on it. For pure states $\rho(\theta)=|\psi(\theta)\rangle\langle\psi(\theta)|$, the quantum Fisher information reduces to the overlap of the derivative of the state $\left|\partial_{\theta} \psi(\theta)\right\rangle$ with itself and the original state (Braunstein, Caves, and Milburn, 1996; Paris, 2009),

$$
I_{\theta}\left(\left|\psi_{\theta}\right\rangle \mid\left\langle\psi_{\theta}\right|\right)=4\left(\left\langle\partial_{\theta} \psi_{\theta} \mid \partial_{\theta} \psi_{\theta}\right\rangle+\left\langle\partial_{\theta} \psi_{\theta} \mid \psi_{\theta}\right\rangle^{2}\right)
$$

If the parameter is imprinted on a pure state via a unitary transformation with Hermitian generator $G$ as $\left|\psi_{\theta}\right\rangle=$ $\exp (i \theta G)|\psi\rangle$, Eq. (13) gives $I_{\theta}=4 \operatorname{Var}(G) \equiv 4\left(\left\langle\psi_{\theta}\left|G^{2}\right| \psi_{\theta}\right\rangle-\right.$ $\left.\left\langle\psi_{\theta}|G| \psi_{\theta}\right\rangle^{2}\right)$. With a maximally entangled state of the $N$ 
subsystems and a suitable measurement, one can reach a scaling of the quantum Fisher information proportional to $N^{2}$ (Giovannetti, Lloyd, and Maccone, 2006), the mentioned Heisenberg limit. This can be seen most easily for a pure state of the form $|\psi\rangle=\left(|\Lambda\rangle^{\otimes N}+|\lambda\rangle^{\otimes N}\right) / \sqrt{2}$, where $|\Lambda\rangle$ and $|\lambda\rangle$ are two eigenstates of $G$ to two different eigenvalues $\Lambda$ and $\lambda$.

For mixed states, the Bures distance is in general difficult to calculate, but $I_{\theta}(\rho(\theta))$ is a convex function of $\rho(\theta)$, i.e., for two density matrices $\rho(\theta)$ and $\sigma(\theta)$ and $0 \leq \lambda \leq 1$ (Fujiwara, 2001a)

$I_{\theta}(\lambda \rho(\theta)+(1-\lambda) \sigma(\theta)) \leq \lambda I_{\theta}(\rho(\theta))+(1-\lambda) I_{\theta}(\sigma(\theta))$.

This can be used to obtain an upper bound for the quantum Fisher information. Convexity also implies that the precision of measurements cannot be increased by classically mixing states with mixing probabilities independent of the parameter (Braun, 2010).

In principle, the optimal measurement that saturates the quantum Cramér-Rao bound can be constructed by diagonalizing $L(\theta)$. The projectors onto its eigenstates form a POVM that yields the optimal measurement. However, such a construction requires that the precise value of the parameter $\theta$ is already known. If that were the case, one could skip the measurement altogether and choose the estimator as $\theta_{\text {est }}=\theta$, with vanishing uncertainty, i.e., apparently violating the quantum Cramér-Rao bound in most cases (ChapeauBlondeau, 2015) (note, however, that for a state that depends on $\theta$, the condition $\theta_{\text {est }}=\theta$ for an unbiased estimator cannot be fulfilled in a whole $\epsilon$ interval about $\theta$, such that there is no formal contradiction). If $\theta$ is not known, the more common approach is therefore to use the quantum Cramér-Rao bound as a benchmark as a function of $\theta$, and then check whether physically motivated measurements can achieve it. More general schemes have been proposed to mitigate the problem of prior knowledge of the parameter. This includes the van Trees inequality (Gill and Levit, 1995; van Trees, 2001), Bayesian approaches (Rivas and Luis, 2012; Macieszczak, Demkowicz-Dobrzański, and Fraas, 2014), adaptive measurements (Wiseman, 1995; Berry and Wiseman, 2000, 2002, 2006, 2013; Armen et al., 2002; Fujiwara, 2006; Higgins et al., 2009; Wheatley et al., 2010; Okamoto et al., 2012; Serafini, 2012), and approaches specialized to particular parameter estimation problems such as phase estimation (Hall et al., 2012). Another point to be kept in mind is that the quantum Cramér-Rao bound can be reached asymptotically for a large number of measurements, but not necessarily for a finite number of measurements. The latter case is clearly relevant for experiments and the subject of active current research (Liu and Yuan, 2016). These limitations not withstanding, this review is based almost exclusively on the quantum Cramér-Rao bound (with the exception of Sec. II.G on quantum channel discrimination and parts of Sec. IV.B.1, where a signal-to-noise ratio is used), given that the overwhelming majority of results have been obtained for it and allow an in-depth comparison of different strategies. A certain number of results have been obtained as well for the quantum Fisher information optimized over all input states (Fujiwara, 2001; Fujiwara and Imai, 2003), a quantity sometimes called channel quantum Fisher information. We do not review this literature here as in this type of work sensitivity is typically not separately optimized over entangled or nonentangled initial states.

The Fisher information can be generalized to multiparameter estimation (Helstrom, 1969; Paris, 2009), $\boldsymbol{\theta}=\left(\theta_{1}, \theta_{2}, \ldots\right)$. The Bures distance between two infinitesimal close states then reads

$$
d s_{\text {Bures }}^{2}\left(\rho_{\boldsymbol{\theta}}, \rho_{\boldsymbol{\theta}+d \boldsymbol{\theta}}\right)=2\left(1-\operatorname{tr} \sqrt{\sqrt{\rho_{\boldsymbol{\theta}}} \rho_{\boldsymbol{\theta}+d \boldsymbol{\theta}} \sqrt{\rho_{\boldsymbol{\theta}}}}\right) .
$$

An expansion of $d s_{\text {Bures }}^{2}\left(\rho_{\boldsymbol{\theta}}, \rho_{\boldsymbol{\theta}+d \boldsymbol{\theta}}\right)$ leads to the quantum Fisher information matrix (Sommers and Zyczkowski, 2003; Paris, 2009),

$$
d s_{\text {Bures }}^{2}\left(\rho_{\boldsymbol{\theta}}, \rho_{\boldsymbol{\theta}+d \boldsymbol{\theta}}\right)=\frac{I_{\theta_{k}, \theta_{k^{\prime}}}}{4} d \theta_{k} d \theta_{k^{\prime}},
$$

where $I_{\theta_{k}, \theta_{k^{\prime}}}=\operatorname{tr} \rho_{\theta}\left(L_{\theta_{k}} L_{\theta_{k^{\prime}}}+L_{\theta_{k}^{\prime}} L_{\theta_{k}}\right) / 2$, and $L_{\theta_{k}}$ is the symmetric logarithmic derivative with respect to parameter $\theta_{k}$. The quantum Cramér-Rao bound generalizes to a lower bound on the covariance matrix $\operatorname{Cov}[\boldsymbol{\theta}]$ of the parameters $\theta_{i}$ (Helstrom, 1969, 1976; Paris, 2009),

$$
\operatorname{Cov}[\boldsymbol{\theta}] \geq \frac{1}{M}[I(\boldsymbol{\theta})]^{-1}
$$

where $\operatorname{Cov}[\boldsymbol{\theta}]_{i j}=\left\langle\theta_{i} \theta_{j}\right\rangle-\left\langle\theta_{i}\right\rangle\left\langle\theta_{j}\right\rangle$, and $A \geq B$ means that $A-B$ is a positive-semidefinite matrix. Contrary to the single parameter quantum Cramér-Rao bound, the bound (17) can in general not be saturated, even in the limit of infinitely many measurements. The Bures metric has also been called fidelity susceptibility in the framework of quantum phase transitions (Gu, 2010).

\section{QUANTUM CORRELATIONS BEYOND ENTANGLEMENT}

\section{A. Parallel versus sequential strategies in unitary quantum metrology}

One of the most typical applications of quantum metrology is the task of unitary parameter estimation, exemplified, in particular, by phase estimation (Giovannetti, Lloyd, and Maccone, 2006, 2011). Let $U_{\theta}=\exp (-i \theta H)$ be a unitary transformation, with $\theta$ the unknown parameter to be estimated, and $H$ a self-adjoint Hamiltonian operator which represents the generator of the transformation. The typical estimation procedure then consists of the following steps: (a) preparing an input probe in a state $\rho$, (b) propagating the state with the unitary transformation $U_{\theta}$, (c) measuring the output state $\rho_{\theta}=U_{\theta} \rho U_{\theta}^{\dagger}$, and (d) performing classical data analysis to infer an estimator $\theta_{\text {est }}$ for the parameter $\theta$.

Assume now that one has the availability of $N$ utilizations of the transformation $U_{\theta}$. Then the use of $N$ uncorrelated probes in a global initial state $\rho^{\otimes N}$, each of which is undergoing the transformation $U_{\theta}$ in parallel, yields an estimator 
whose minimum variance scales as $1 / N$ (standard quantum limit). On the other hand, by using an initial entangled state $\rho$ of the $N$ probes, and propagating each with the unitary $U_{\theta}$ in parallel, one can in principle achieve the Heisenberg limit, meaning that an optimal estimator $\theta_{\text {est }}$ can be constructed whose asymptotic variance, in the limit $N \gg 1$, scales as $1 / N^{2}$. However, it is not difficult to realize that the very same precision can be reached without the use of entanglement, by simply preparing a single input probe in a superposition state with respect to the eigenbasis of the generator $H$, and letting the probe undergo $N$ sequential iterations of the transformation $U_{\theta}$.

For instance, thinking of each probe as a qubit for simplicity, and fixing the generator $H$ to be the Pauli matrix $\sigma_{z}$, one can consider either a parallel scheme with $N$ input probes in the Greenberger-Horne-Zeilinger (GHZ, or catlike) maximally entangled state $|\Psi\rangle=(|00 \cdots 0\rangle+|11 \cdots 1\rangle) / \sqrt{2}$ or a sequential scheme with a single probe in the superposition $|\psi\rangle=(|0\rangle+|1\rangle) / \sqrt{2}$. In the first case, the state after imprinting the parameter reads $U_{\theta}^{\otimes N}|\Psi\rangle=\left(e^{-i N \theta}|00 \cdots 0\rangle+\right.$ $\left.e^{i N \theta}|11 \cdots 1\rangle\right) / \sqrt{2}$, while in the second case $U_{\theta}^{N}|\psi\rangle=$ $\left(e^{-i N \theta}|0\rangle+e^{i N \theta}|1\rangle\right) / \sqrt{2}$. Hence, in both schemes one achieves an $N$-fold increase of the phase between two orthogonal states, and both schemes reach the Heisenberglimit scaling in the estimation of the phase shift $\theta$, meaning that the quantum Cramér-Rao bound can be asymptotically saturated in both cases by means of an optimal measurement, associated with a quantum Fisher information scaling quadratically with $N$. The equivalence between entanglement in parallel schemes and coherence (namely, superposition in the eigenbasis of the generator) (Baumgratz, Cramer, and Plenio, 2014; Marvian and Spekkens, 2016; Streltsov, Adesso, and Plenio, 2017) in sequential schemes further extends to certain schemes of quantum metrology in the presence of noise, namely, when the unitary encoding the parameter to be estimated and the noisy channel commute with each other (e.g., in the case of phase estimation affected by dephasing) (Boixo and Heunen, 2012; Demkowicz-Dobrzański and Maccone, 2014), although in more general instances entanglement is shown to provide an advantage (Huelga et al., 1997; Escher, de Matos Filho, and Davidovich, 2011; Demkowicz-Dobrzański and Maccone, 2014). In general, sequential schemes such that individual probes are initially correlated with an ancilla (on which the parameter is not imprinted) and assisted by feedback control (see Sec. IV.F.4) can match or outperform any parallel scheme for estimation of single or multiple parameters encoded in unitary transformations even in the presence of noise (Demkowicz-Dobrzański and Maccone, 2014; Yuan and Fung, 2015; Huang, Macchiavello, and Maccone, 2016; Nichols et al., 2016; Yuan, 2016; Sekatski et al., 2017; Yousefjani et al., 2017). While probe and ancilla typically need to be entangled for such sequential schemes to achieve maximum quantum Fisher information, this observation removes the need for large-scale multiparticle entangled probes in the first place.

Similarly, in continuous-variable optical interferometry (Caves, 1981), equivalent performances can be reached (for unitary phase estimation) by using either a two-mode entangled probe, such as a NOON state, or a single-mode nonclassical state, such as a squeezed state. These are elementary examples of quantum-enhanced measurements achievable without entanglement, yet exploiting genuinely quantum effects such as nonclassicality and superposition. Such features can be understood by observing that both optical nonclassicality in infinite-dimensional systems and coherence (superposition) in finite-dimensional systems can be converted to entanglement within a well-defined resourcetheoretic framework (Asbóth, Calsamiglia, and Ritsch, 2005; Vogel and Sperling, 2014; Streltsov et al., 2015; Killoran, Steinhoff, and Plenio, 2016), and can be thought of as equivalent resources to entanglement for certain practical purposes, as is evidently the case for unitary metrology. ${ }^{1}$

\section{B. General results on the usefulness of entanglement}

More generally, for unitary metrology with multipartite probes in a parallel setting, a quite general formalism has been developed to identify the metrologically useful correlations in the probes in order to achieve quantum-enhanced measurements (Pezzè and Smerzi, 2009) [see Pezzè and Smerzi (2014) and Tóth and Apellaniz (2014) for recent reviews]. Specifically, let us consider an input state $\rho$ of $N$ qubits and a linear interferometer with Hamiltonian generator given by

$$
H=J_{l}=\frac{1}{2} \sum_{i=1}^{N} \sigma_{l}^{(i)},
$$

i.e., a component of the collective (pseudo-)angular momentum of the $N$ probes in the direction $l=x, y, z$, with $\sigma_{l}^{(i)}$ denoting the $l$ th Pauli matrix for qubit $i$. If $\rho$ is $k$ producible, i.e., it is a convex mixture of pure states which are tensor products of at most $k$-qubit states, then the quantum Fisher information is bounded above as follows (Hyllus et al., 2012; Tóth, 2012),

$$
I_{\theta}\left(\rho, J_{l}\right) \leq n k^{2}+(N-n k)^{2},
$$

where $n$ is the integer part of $N / k$. This means that genuine multipartite entangled probes $(k=N)$ are required to reach the maximum sensitivity, given by the Heisenberg limit $I_{\theta} \propto N^{2}$, even though partially entangled states can still result in quantum-enhanced measurements beyond the standard quantum limit.

A similar conclusion was reached by Augusiak et al. (2016) considering the geometric measure of entanglement, which quantifies how far $\rho$ is from the set of fully separable (1producible) states according to the fidelity. Namely, for unitary metrology with $N$ parallel probes initialized in the mixed state $\rho$, in the limit $N \rightarrow \infty$ a nonvanishing value of the

\footnotetext{
${ }^{1}$ An additional scenario in which the quantum limit can be reached without entanglement is when a multipartite state is used to measure multiple parameters, where each parameter is encoded locally onto only one subsystem-it has recently been shown that entanglement between the subsystems is not advantageous, and can even be detrimental, in this setting (Knott et al., 2016; Proctor, Knott, and Dunningham, 2018).
} 
geometric measure of entanglement of $\rho$ is necessary for the exact achievement of the Heisenberg limit. However, a sensitivity arbitrarily close to the Heisenberg limit $I_{\theta} \propto$ $N^{2-\epsilon}$ for any $\epsilon>0$ can still be attained even if the geometric measure of entanglement of $\rho$ vanishes asymptotically for $N \rightarrow \infty$. In deriving these results, they proved an important continuity relation for the quantum Fisher information in unitary dynamics (Augusiak et al., 2016).

\section{Role of quantum discord in parameter estimation with mixed probes}

This section focuses on possible advantages stemming from the use of quantum correlations more general than entanglement in the (generally mixed) state of the input probes for a metrological task. Such correlations are usually referred to under the collective name of quantum discord (Henderson and Vedral, 2001; Ollivier and Zurek, 2001), see also Modi et al. (2012) and Adesso, Bromley, and Cianciaruso (2016) for recent reviews. The name quantum discord originates from a mismatch between two possible quantum generalizations of the classical mutual information, a measure of correlations between two (or more) variables described by a jointprobability distribution (Ollivier and Zurek, 2001). A direct generalization leads to the so-called quantum mutual information $I(\rho)=S\left(\operatorname{Tr}_{A} \rho\right)+S\left(\operatorname{Tr}_{B} \rho\right)-S(\rho)$ that quantifies total correlations in the state $\rho$ of a bipartite system $A B$, with $S(\rho)=-\operatorname{Tr}(\rho \log \rho)$ being the von Neumann entropy. An alternative generalization leads instead to $J^{(A)}(\rho)=$ $\sup _{\left\{\Pi^{A}\right\}} I\left(\Pi^{A}[\rho]\right)$, a measure of one-sided classical correlations that quantifies how much the marginal entropy of, say, subsystem $B$ is decreased (i.e., how much additional information is acquired) by performing a minimally disturbing measurement on subsystem $A$ described by a POVM $\left\{\Pi^{A}\right\}$, with $\Pi^{A}[\rho]$ being the conditional state of the system $A B$ after such measurement (Henderson and Vedral, 2001). The difference between the former and the latter quantity is precisely the quantum discord

$$
D^{(A)}(\rho)=I(\rho)-J(\rho)
$$

that quantifies therefore just the quantum portion of the total correlations in the state $\rho$ from the perspective of subsystem $A$. It is clear from this definition that the state $\rho$ of a bipartite system $A B$ has nonzero discord (from the point of view of $A$ ) if and only if it is altered by all possible local measurements performed on subsystem $A$ : disturbance by measurement is a genuine quantum feature which is captured by the concept of discord; see Modi et al. (2012) and Adesso, Bromley, and Cianciaruso (2016) for more details. Every entangled state is also discordant, but the converse is not true; in fact, almost all separable states still exhibit nonzero discord (Ferraro et al., 2010). The only bipartite states with zero discord, from the point of view of subsystem $A$, are so-called classical-quantum states, which take the form

$$
\chi^{(A)}=\sum_{i} p_{i}|i\rangle\left\langle\left. i\right|^{A} \otimes \tau_{i}^{B}\right.
$$

where the states $\left\{|i\rangle^{A}\right\}$ form an orthonormal basis for subsystem $A$, and $\left\{\tau_{i}^{B}\right\}$ denote a set of arbitrary states for subsystem $B$, while $\left\{p_{i}\right\}$ stands for a probability distribution. These states are left invariant by measuring $A$ in the basis $\left\{|i\rangle^{A}\right\}$, which entails that $D^{(A)}\left(\chi^{(A)}\right)=0$.

In a multipartite setting, one can define fully classical states as the states with zero discord with respect to all possible subsystems, or alternatively as the states which are left invariant by a set of local measurements performed on all subsystems. Such states take the form $\chi=\sum_{i_{1}, \ldots, i_{N}} p_{i_{1}, \ldots, i_{N}}\left|i_{1}\right\rangle\left\langle\left. i_{1}\right|^{A_{1}} \otimes\right.$ $\cdots \otimes\left|i_{N}\right\rangle\left\langle\left. i_{N}\right|^{A_{N}}\right.$ for an $N$-particle system $A_{1} \cdots A_{N}$; i.e., they are diagonal in a local product basis. One can think of these states as the only ones which are completely classically correlated, that is, completely described by a classical multivariate probability distribution $\left\{p_{i_{1}, \ldots, i_{N}}\right\}$, embedded into a density matrix formalism. An alternative way to quantify discord in a (generally multipartite) state $\rho$ is then by taking the distance between $\rho$ and the set of classically correlated states, according to a suitable (quasi)distance function. For instance, the relative entropy of discord (Modi et al., 2010) is defined as

$$
D_{R}(\rho)=\inf _{\chi} S(\rho \| \chi)
$$

where the minimization is over all classically correlated states $\chi$, and $S(\rho \| \chi)=\operatorname{Tr}(\rho \log \rho-\rho \log \chi)$ denotes the quantum relative entropy. For a dedicated review on different measures of discord-type quantum correlations see Adesso, Bromley, and Cianciaruso (2016).

Regarding the role of quantum discord in metrological contexts, Modi et al. (2011) investigated the estimation of a unitary phase $\theta$ applied to each of $N$ qubit probes, initially prepared in mixed states with either (a) no correlations, (b) only classical correlations, or (c) quantum correlations (discord and/or entanglement). All the considered families of $N$-qubit probe states were chosen with the same spectrum, i.e., in particular, the same degree of mixedness (which is a meaningful assumption if one is performing a metrology experiment in an environment with a fixed common temperature), and were selected due to their relevance in recent nuclear magnetic resonance (NMR) experiments (Jones et al., 2009). In particular, given an initial thermal state

$$
\rho_{0}(p)=\left(\frac{1+p}{2}|0\rangle\left\langle 0\left|+\frac{1-p}{2}\right| 1\right\rangle\langle 1|\right)
$$

for each single qubit (with purity parameter $0 \leq p \leq 1$ ), the product states $\rho_{N}^{(\mathrm{a})}(p)=\left[H \rho_{0}(p) H\right]^{\otimes N}$ were considered for case (a), and the GHZ-diagonal states $\rho_{N}^{(\mathrm{c})}(p)=$ $C \mathrm{H}_{1} C \rho_{0}(p)^{\otimes N} \mathrm{CH}_{1} \mathrm{C}$ were considered for case (c), with $H$ denoting the single-qubit Hadamard gate (acting on each qubit in the first case, and only on the first qubit in the second case), and $C=\otimes_{j=2}^{N}$ C-NOT $_{1 j}$ a series of CONTROL-NOT operations acting on pairs of qubits 1 and $j$. These two classes of states give rise to quantum Fisher information $I_{\theta}^{(\mathrm{a})}=p^{2} N$ and $I_{\theta}^{(\mathrm{c})} \gtrsim p^{2} N^{2}$, respectively. By comparing the two cases, Modi et al. (2011) concluded that a quantum enhancement, scaling 
as $I_{\theta}^{(\mathrm{c})} / I_{\theta}^{(\mathrm{a})} \approx N$, is possible using pairs of mixed probe states with arbitrary (even infinitesimally small) degree of purity. This advantage persists even when the states in strategy (c) are fully separable, which occurs for $p \lesssim a+b / N$ (with $a$ and $b$ determined numerically for each value of $N$ ), in which case both strategies are unable to beat the standard quantum limit, yet the quadratic enhancement of (c) over (a) is maintained, being independent of $p$. They then argued that multipartite quantum discord, which increases with $N$ according to the relative entropy measure of Eq. (21) and vanishes only at $p=0$, may be responsible for this enhancement. Notice that, even though the quantum Fisher information is convex (which means that for every separable but discordant mixed state there exists a pure product state with a higher or equal quantum Fisher information), the analysis in Modi et al. (2011) was performed at fixed spectrum (and thus degree of purity) of the input probes, a constraint which allowed them to still identify an advantage in using correlations weaker than entanglement, as opposed to no correlations. However, it is presently unclear whether these conclusions are special to the selected classes of states or whether they can be further extended to more general settings, including noisy metrology.

In a more recent work, Cable, Gu, and Modi (2016) analyzed a model of unitary quantum metrology inspired by the computational algorithm known as deterministic quantum computation with one quantum bit (DQC1) or "power of one qubit" (Knill and Laflamme, 1998). Using only one pure qubit supplemented by a register of $l$ maximally mixed qubits, all individually subject to a local unitary phase shift $U_{\theta}$, their model was shown to achieve the standard quantum limit for the estimation of $\theta$, which can be conventionally obtained using the same number of qubits in pure uncorrelated states. They found that the standard quantum limit can be exceeded by using one additional qubit, which contributes only a small degree of extra purity, which, however, for any finite amount of extra purity leads to an entangled state at the stage of parameter encoding. In this model, incidentally, the output state after the unitary encoding was found to be always separable but discordant, with its discord vanishing only in the limit of vanishing variance of the estimator for the parameter $\theta$. It is not quite clear if and how the discord in the final state can be interpreted in terms of a resource for metrology, but the achievement of the standard quantum limit with all but one probes in a fully mixed state was identified as a quantum enhancement without the use of entanglement. This suggests that further investigation on the role of quantum discord (as well as state purity) in metrological algorithms with vanishing entanglement may be in order. The protocol for multiparameter estimation using DQC1 was studied by Boixo and Somma (2008), although the resource role of correlations was not discussed there. MacCormick et al. (2016) made a detailed investigation of a DQC1-based protocol based on coherently controlled Rydberg interactions between a single atom and an atomic ensemble containing $N$ atoms. The protocol allows one to estimate a phase shift assumed identical for all atoms in the atomic ensemble with a sensitivity that interpolates smoothly between the standard quantum limit and the Heisenberg limit when the purity of the atomic ensemble increases from a fully mixed state to pure states. It leads to a cumulative phase shift proportional to $N$, and the scheme can in fact also be seen as an implementation of "coherent averaging," with the control qubit playing the role of the "quantum bus" (see Sec. IV.F.3).

\section{Black-box metrology and the interferometric power}

As explicitly discussed in Sec. II.A, for unitary parameter estimation, if one has full prior information on the generator $H$ of the unitary transformation $U_{\theta}$ imprinting the parameter $\theta$, then no correlations are required whatsoever, and probe states with coherence in the eigenbasis of $H$ suffice to achieve quantum-enhanced measurements in a sequential scheme. Recently, Girolami, Tufarelli, and Adesso (2013), Adesso (2014), and Girolami et al. (2014) investigated quantum metrology in a so-called black-box paradigm, according to which the generator $H$ is assumed not fully known a priori. In such a case, suppose one selects a fixed (but arbitrary) input single-particle probe $\rho$, then it is impossible to guarantee a precision in the estimation of $\theta$ for all possible nontrivial choices of $H$. This is because, in the worst case scenario, the black-box unitary transformation may be generated by a $H$ which commutes with the input state $\rho$, resulting in no information imprinted on the probe, hence in a vanishing quantum Fisher information. It is clear then that, to be able to estimate parameters independently of the choice of the generator, one needs an ancillary system correlated with the probe. But what type of correlations are needed? It is in this context that discord-type correlations, rather than entanglement or classical correlations, are found to play a key resource role.

Consider a standard two-arm interferometric configuration, and let us retrace the steps of parameter estimation in the black-box scenario (Girolami et al., 2014): (a) an input state $\rho$ of two particles, the probe $A$ and the ancilla $B$, is prepared; (b) particle $B$ is transmitted with no interaction, while particle $A$ enters a black box where it undergoes a unitary transformation $U_{\theta}=\exp (-i \theta H)$ generated by a Hamiltonian $H$, whose spectrum is known but whose eigenbasis is unknown at this stage; (c) the agent controlling the black box announces the full specifics of the generator $H$, so that parties $A$ and $B$ can jointly perform the best possible measurement on the two-particle output state $\rho_{\theta}=\left(U_{\theta} \otimes \mathbb{I}\right) \rho\left(U_{\theta} \otimes \mathbb{I}\right)^{\dagger}$; and (d) the whole process is iterated $N$ times, and an optimal unbiased estimator $\theta_{\text {est }}$ is eventually constructed for the parameter $\theta$. In the limit $N \gg 1$, for any specific black-box setting $H$, the corresponding quantum Fisher information $I_{\theta}(\rho, H)$ determines the maximal precision enabled by the input state $\rho$ in estimating the parameter $\theta$ generated by $H$, as prescribed by the quantum Cramér-Rao bound.

One can then introduce a figure of merit quantifying the worst case precision guaranteed by the state $\rho$ for the estimation of $\theta$ in this black-box protocol. This is done naturally by minimizing the quantum Fisher information over all generators $H$ within the given spectral class (the spectrum is assumed nondegenerate, with a canonical choice being that of equispaced eigenvalues) (Girolami, Tufarelli, and Adesso, 2013; Girolami et al., 2014). This defines (up to a 
normalization constant) the interferometric power of the bipartite state $\rho$ with respect to the probing system $A$,

$$
P^{(A)}(\rho)=\frac{1}{4} \min _{H} I_{\theta}(\rho, H) .
$$

Remarkably, as proven by Girolami et al. (2014), the interferometric power turns out to be a measure of discordtype correlations in the input state $\rho$. In particular, it vanishes if and only if $\rho$ takes the form of a classical-quantum state, Eq. (20). This entails that states with zero discord cannot guarantee a precision in parameter estimation in the worst case scenario, while any other bipartite state (entangled or separable) with nonzero discord is suitable for estimating parameters encoded by a unitary transformation (acting on one subsystem) no matter the generator, with minimum guaranteed precision quantified by the interferometric power of the state. This conclusion holds both for parameter estimation in finitedimensional systems (Girolami et al., 2014) and for continuous-variable optical interferometry (Adesso, 2014). Recently, it was shown more formally that entanglement accounts only for a portion of the quantum correlations relevant for bipartite quantum interferometry. In particular, the interferometric power, which is by definition a lower bound to the quantum Fisher information (for any fixed generator $H$ ), is itself bounded from below in bipartite systems of any dimension by a measure of entanglement aptly named the interferometric entanglement, which simply reduces to the squared concurrence for two-qubit states (Bromley et al., 2017). The interferometric power can be evaluated in closed form, analytically solving the minimization in Eq. (22), for all finite-dimensional states such that subsystem $A$ is a qubit (Girolami et al., 2014), and for all two-mode Gaussian states when the minimization is restricted to Gaussian unitaries (Adesso, 2014). An experimental demonstration of black-box quantum-enhanced measurements relying on discordant states as opposed to classically correlated states has been reported using a two-qubit NMR ensemble realized in chloroform (Girolami et al., 2014).

Notice finally that, while quantum correlations with an ancilla are required to achieve a nonzero worst case precision when minimizing the quantum Fisher information over the choice of the generator $H$ within a fixed spectral class, as in the scenario considered here, single-probe (nonmaximally mixed) states may however suffice to be useful resources in the arguably more practical case in which the average precision, rather than the minimal, is considered instead as a figure of merit. This scenario is further discussed in Sec. II.H.

\section{E. Quantum estimation of bosonic loss}

Any quantum optical communication, from fiber-based to free-space implementations, is inevitably affected by energy dissipation. The fundamental model to describe this scenario is the lossy channel. This attenuates an incoming bosonic mode by transmitting a fraction $\eta \leq 1$ of the input photons, while sending the other fraction $1-\eta$ into the environment. The maximum number of bits per channel use at which we can transmit quantum information, distribute entanglement, or generate secret keys through such a lossy channel are all equal to $-\log (1-\eta)$ (Pirandola et al., 2017), a fundamental rateloss tradeoff that only quantum repeaters may surpass (Pirandola, 2016). For these and other implications to quantum communication, it is of paramount importance to estimate the transmissivity of a lossy channel in the best possible way.

Quantum estimation of bosonic loss was first studied by Monras and Paris (2007) by using single-mode pure Gaussian states [see also Pinel et al. (2013)]. In this setting, the performance of the coherent state probes at fixed input energy provides the shot-noise limit or classical benchmark, which has to be beaten by truly quantum probes. Let us denote by $\bar{n}$ the mean number of input photons, then the shot-noise limit is equal to $I_{\eta} \simeq \eta^{-1} \bar{n}$ (Monras and Paris, 2007; Pinel et al., 2013). The use of squeezing can beat this limit, following the original intuition for phase estimation of Caves (1981). In fact, Monras and Paris (2007) showed that, in the regime of small loss $\eta \simeq 1$ and small energy $\bar{n} \simeq 0$, a squeezed vacuum state can beat the standard quantum limit. The use of squeezing for estimating the interaction parameter in bilinear bosonic Hamiltonians (including beam-splitter interactions) was also discussed by Gaiba and Paris (2009), showing that unentangled single-mode squeezed probes offer equivalent performance to entangled two-mode squeezed probes for practical purposes.

The optimal scaling $I_{\eta} \simeq[\eta(1-\eta)]^{-1} \bar{n}$ can be achieved by using Fock states at the input (Adesso et al., 2009). Note that, because Fock states can be used only when the input energy corresponds to integer photon numbers, in all the other cases one needs to engineer superpositions, e.g., between the vacuum and the one-photon Fock state if we want to explore the low-energy regime $\bar{n} \lesssim 1$. Non-Gaussian qutrit and quartet states can be designed to beat the best Gaussian probes (Adesso et al., 2009). It is still an open question to determine the optimal probes for estimating loss at any energy regime. It is certainly known that the bound $I_{\eta} \leq[\eta(1-\eta)]^{-1} \bar{n}$ holds for any $\bar{n}$, as it can be proven by dilating the lossy channel into a beam-splitter unitary and then performing parameter estimation (Monras and Paris, 2007). Note that this bound is computed by considering $N$ uncorrelated probes in parallel. It is therefore an open question to find the best performance that is achievable by the most general (adaptive) strategies.

Interestingly, the problem of estimating the loss parameters of a pair of lossy bosonic channels has been proven formally equivalent to the problem of estimating the separation of two incoherent optical pointlike sources (Lupo and Pirandola, 2016). In this context Tsang, Nair, and Lu (2016) showed that a pair of weak thermal sources can be resolved independently from their separation if one adopts quantum measurements based on photon counting, instead of standard intensity measurements. Thus, quantum detection strategies enable one to beat the so-called "Rayleigh's curse" which affects classical imaging (Tsang, Nair, and Lu, 2016). This curse is reinstated in the classical limit of very bright thermal sources (Lupo and Pirandola, 2016; Nair and Tsang, 2016). On the other hand, Lupo and Pirandola (2016) showed that quantumcorrelated sources can be superresolved at the sub-Rayleigh scale. In fact, it is possible to engineer quantum-correlated pointlike sources that are not entangled (but discordant) which displays superresolution, so that the closer the sources are the better their distance can be estimated. 
The estimation of loss becomes complicated in the presence of decoherence, such as thermal noise in the environment and nonunit efficiency of the detectors. From this point of view, Spedalieri et al. (2016) considered a very general model of Gaussian decoherence which also includes the potential presence of non-Markovian memory effects. In such a scenario, Spedalieri et al. (2016) showed the utility of asymmetrically correlated thermal states (i.e., with largely different photon numbers in the two modes), fully based on discord and void of entanglement. These states can be used to estimate bosonic loss with a sensitivity that approaches the shot noise limit and may also surpass it in the presence of correlated noise and memory effects in the environment. This kind of thermal quantum metrology has potential applications for practical optical instruments (e.g., photometers) or at different wavelengths (e.g., far infrared, microwave or $\mathrm{x}$ ray) for which the generation of quantum features, such as coherence, number states, squeezing, or entanglement, may be challenging.

\section{F. Gaussian quantum metrology}

Clearly one may also consider the estimation of other parameters beyond loss. In general, Gaussian quantum metrology aims at estimating any parameter or multiple parameters encoded in a bosonic Gaussian channel. As shown by Pirandola and Lupo (2017), the most general adaptive estimation of noise parameters (such as thermal or additive noise) cannot beat the standard quantum limit. This is because Gaussian channels are teleportation covariant, i.e., they suitably commute with the random operations induced by quantum teleportation, a property which is shared by a large class of quantum channels at any dimension (Pirandola et al., 2017). The joint estimation of specific combinations of parameters, such as loss and thermal noise, or the two real components of a displacement, has been widely studied in the literature (Bellomo et al., 2009, 2010a, 2010b; Monras and Illuminati, 2011; Genoni et al., 2013; Gao and Lee, 2014; Gagatsos, Branford, and Datta, 2016; Duivenvoorden, Terhal, and Weigand, 2017), but the ultimate performance achievable by adaptive (i.e., feedback-assisted) schemes is still unknown.

If one employs Gaussian states at the input of a Gaussian channel, then one has Gaussian states at the output and one may exploit closed formulas for the quantum Fisher information. These formulas can be derived by direct evaluation of the symmetric logarithmic derivative (Monras, 2013; Jiang, 2014; Šafránek, Lee, and Fuentes, 2015; Nichols et al., 2017) or by considering the infinitesimal expression of the quantum fidelity (Pinel et al., 2012, 2013; Banchi, Braunstein, and Pirandola, 2015). The latter approach may exploit general and handy formulas. In fact, for two arbitrary multimode Gaussian states $\rho_{1}$ and $\rho_{2}$ with mean values $u_{1}$ and $u_{2}$, and covariance matrices $V_{1}$ and $V_{2}$, one may write their quantum fidelity (Banchi, Braunstein, and Pirandola, 2015)

$$
\begin{aligned}
& F\left(\rho_{1}, \rho_{2}\right)=\frac{F_{\text {tot }} e^{-(1 / 2)\left(u_{2}-u_{1}\right)^{T}\left(V_{1}+V_{2}\right)^{-1}\left(u_{2}-u_{1}\right)}}{\sqrt{\operatorname{det}\left(V_{1}+V_{2}\right)}}, \\
& F_{\text {tot }}^{2}=\operatorname{det}\left[2\left(\sqrt{\mathbb{1}+\frac{\left(V_{\text {aux }} \Omega\right)^{-2}}{4}}+\mathbb{1}\right) V_{\text {aux }}\right],
\end{aligned}
$$

where $V_{\text {aux }}:=\Omega^{T}\left(V_{1}+V_{2}\right)^{-1}\left(\Omega / 4+V_{2} \Omega V_{1}\right)$ with $\Omega$ being the symplectic form. Specific expressions for the fidelity were previously given for single-mode Gaussian states (Scutaru, 1998), two-mode Gaussian states (Marian and Marian, 2012), multimode Gaussian states assuming that one of the states is pure (Spedalieri, Weedbrook, and Pirandola, 2013), and multimode thermal states with vanishing first moments (Paraoanu and Scutaru, 2000).

From Eq. (23) one may derive the Bures metric $d s_{\text {Bures. In }}^{2}$ fact, consider two infinitesimally close Gaussian states $\rho$, with statistical moments $u$ and $V$, and $\rho+d \rho$, with statistical moments $u+d u$ and $V+d V$. Expanding at the second order in $d u$ and $d V$, one finds (Banchi, Braunstein, and Pirandola, 2015)

$$
d s_{\text {Bures }}^{2}=2[1-\sqrt{F(\rho, \rho+d \rho)}]=\frac{d u^{T} V^{-1} d u}{4}+\frac{\delta}{8},
$$

where $\delta:=4 \operatorname{Tr}\left[d V\left(4 \mathcal{L}_{V}+\mathcal{L}_{\Omega}\right)^{-1} d V\right], \mathcal{L}_{A} X:=A X A$, and the inverse of the superoperator $4 \mathcal{L}_{V}+\mathcal{L}_{\Omega}$ refers to the pseudoinverse. A similar expression was also computed by Monras (2013) using the symmetric logarithmic derivative, with further refinements in Šafránek, Lee, and Fuentes (2015). From the Bures metric in Eq. (25) one may derive the quantum Fisher information [see Eq. (12)] for the estimation of any parameter encoded in a multimode (pure or mixed) Gaussian state directly in terms of the statistical moments. Equation (25) is written in a compact basis-independent and parametrization-independent form, valid for any multimode Gaussian state. For an explicit parametrization via multiple parameters $\boldsymbol{\theta}=\left(\theta_{1}, \theta_{2}, \ldots\right)$, one can expand the differential and write $d V=\sum_{k} \partial_{\theta_{k}} V d \theta_{k}$, and similarly for $d u$. In this way, $d s_{\text {Bures }}^{2}=$ $\sum_{k, k^{\prime}}(1 / 4) I_{\theta_{k}, \theta_{k^{\prime}}} d \theta_{k} d \theta_{k^{\prime}}$ as in Eq. (16), with

$$
\begin{aligned}
I_{\theta_{k}, \theta_{k^{\prime}}}= & \left(\partial_{\theta_{k}} u^{T}\right) V^{-1}\left(\partial_{\theta_{k^{\prime}}} u\right) \\
& +2 \operatorname{Tr}\left[\left(\partial_{\theta_{k}} V\right)\left(4 \mathcal{L}_{V}+\mathcal{L}_{\Omega}\right)^{-1}\left(\partial_{\theta_{k^{\prime}}} V\right)\right]
\end{aligned}
$$

Equations (25) and (26) have been derived following Eq. (12), namely, explicitly computing the fidelity function for the two most general multimode Gaussian states, and then taking the limit of two infinitesimally close states. A similar approach was used for fermionic Gaussian states in Banchi, Giorda, and Zanardi (2014).

An alternative derivation of the bosonic quantum Fisher information for multimode Gaussian states, based on the use of the symmetric logarithmic derivative, was recently obtained by Nichols et al. (2017). Furthermore, Nichols et al. (2017) derived a necessary and sufficient compatibility condition such that the quantum Cramér-Rao bound Eq. (17) is asymptotically achievable in multiparameter Gaussian quantum metrology, meaning that a single optimal measurement exists which is able to extract the maximal information on all the parameters simultaneously. For any pair of parameters $\theta_{k}$, $\theta_{k^{\prime}} \in \boldsymbol{\theta}$, in terms of the symmetric logarithmic derivatives $L_{\rho_{\theta_{k}}}$ and $L_{\rho_{\theta_{k^{\prime}}}}$, the corresponding quantum Fisher information matrix element is defined as $I_{\theta_{k}, \theta_{k^{\prime}}} \equiv \operatorname{Re}\left[\operatorname{Tr}\left(\rho_{\theta} L_{\rho_{\theta_{k}}} L_{\rho_{\theta_{k^{\prime}}}}\right)\right]$, while the measurement compatibility condition amounts to $Y_{\theta_{k}, \theta_{k^{\prime}}} \equiv \operatorname{Im}\left[\operatorname{Tr}\left(\rho_{\boldsymbol{\theta}} L_{\rho_{\theta_{k}}} L_{\rho_{\theta_{k^{\prime}}}}\right)\right]=0 \quad$ (Ragy, Jarzyna, and 
Demkowicz-Dobrzański, 2016). In terms of the first and second statistical moments $u$ and $V$ of a $m$-mode Gaussian state $\rho_{\boldsymbol{\theta}}$, we then have (Nichols et al., 2017)

$$
\begin{gathered}
I_{\theta_{k}, \theta_{k^{\prime}}}=\left(\partial_{\theta_{k}} u^{T}\right) V^{-1}\left(\partial_{\theta_{k^{\prime}}} u\right)+2 \operatorname{Tr}\left(\partial_{\theta_{k^{\prime}}} V K_{\theta_{k}}\right), \\
Y_{\theta_{k}, \theta_{k^{\prime}}}=\frac{1}{2}\left(\partial_{\theta_{k}} u^{T}\right) V^{-1} \Omega V^{-1}\left(\partial_{\theta_{k^{\prime}}} u\right)+16 \operatorname{Tr}\left(\Omega K_{\theta_{k^{\prime}}} V K_{\theta_{k}}\right),
\end{gathered}
$$

with

$$
K_{\theta}=\sum_{i, j=1}^{m} \sum_{l=0}^{3} \frac{\left(a_{\theta}\right)_{l}^{i j}}{\nu_{i} \nu_{j}-(-1)^{l}} S^{T-1} M_{l}^{i j} S^{-1},
$$

where $\left(a_{\theta}\right)_{l}^{i j}=\operatorname{Tr}\left(S^{-1} \partial_{\theta} V S^{T-1} M_{l}^{i j}\right),\left\{\nu_{i}\right\}$ are the symplectic eigenvalues of the covariance matrix $V, S^{-1}$ is the symplectic transformation that brings $V$ into its diagonal form, $S^{-1} V S^{T-1}=\bigoplus_{i=1}^{m} \nu_{i} \mathbb{1}$, and the set of matrices $M_{l}^{i j}$ have all zero entries except for the $2 \times 2$ block in position $i j$ which is given by $\left\{(M)_{l}^{i j}\right\}_{l \in\{0, \ldots, 3\}}=(1 / \sqrt{2})\left\{i \sigma_{y}, \sigma_{z}, \mathbb{1}, \sigma_{x}\right\}$. Note that Eq. (27) can also be obtained from (25) by explicitly writing all the operators in the basis in which $V$ is diagonal, observing that $\left(4 \mathcal{L}_{V}+\mathcal{L}_{\Omega}\right)^{-1}\left(\partial_{\theta} V\right)=K_{\theta}$. On the other hand, Eq. (28) cannot be obtained from the limit of the fidelity formula.

In the context of this review, Pinel et al. (2012) studied, in particular, the quantum Cramér-Rao bound for estimating a parameter $\theta$ which is encoded in a pure multimode Gaussian state. It was realized that, in the limit of large photon number, no entanglement nor correlations between different modes are necessary for obtaining the optimal sensitivity. Rather, a detection mode can be used based on the derivative of the mean photon field with respect to the parameter $\theta$, into which all the resources in terms of photons and squeezing should be put. The mean photon field is defined as $\bar{a}_{\theta}(\boldsymbol{r}, t)=$ $\left\langle\psi_{\theta}|a(\boldsymbol{r}, t)| \psi_{\theta}\right\rangle$, with all parameter dependence in the pure Gaussian quantum state $|\psi\rangle_{\theta}, a(\boldsymbol{r}, t)=\sum_{i} a_{i} v_{i}(\boldsymbol{r}, t)$, where $v_{i}(\boldsymbol{r}, t)$ are orthonormal mode functions found from solving Maxwell's equation with appropriate boundary conditions, $a_{i}$ is the annihilation operators of mode $i$, and the sum is over all modes. The mean field can be normalized, $u_{\theta}=\bar{a}_{\theta}(\boldsymbol{r}, t) /\left\|\bar{a}_{\theta}\right\|$, where the norm $\|f(\boldsymbol{r}, t)\|=$ $\left[\int|f(\boldsymbol{r}, t)|^{2} d^{2} \boldsymbol{r} d t\right]^{1 / 2}$ contains spatial integration over a surface perpendicular to the light beam propagation and temporal integration over the detection time. The detection mode is then defined as

$$
\tilde{v}_{1}(\boldsymbol{r}, t)=\frac{\bar{a}_{\theta}^{\prime}(\boldsymbol{r}, t)}{\left\|\bar{a}_{\theta}^{\prime}\right\|},
$$

where the prime means derivative with respect to $\theta$. The detection mode can be complemented by other, orthonormal modes to obtain a full basis, but these other modes need not be excited for achieving maximum quantum Fisher information. The quantum Fisher information reads then

$$
I_{\theta}=N_{\theta}\left[4\left\|u_{\theta}^{\prime}\right\|^{2}+\left(\frac{N_{\theta}^{\prime}}{N_{\theta}}\right)^{2}\right] V_{\theta,[1,1]}^{-1}
$$

where $N_{\theta}$ is the mean photon number, and $V_{\theta,[1,1]}^{-1}$ the matrix element of the inverse covariance matrix of the Gaussian state corresponding to the detection mode $\tilde{v}_{1}(\boldsymbol{r}, t)$. All other modes are chosen orthonormal to it. The standard quantum limit corresponds to a quantum Fisher information of a coherent state, in which case $V_{\theta,[1,1]}^{-1}=1$. Hence, an improvement over the standard quantum limit is possible with pure Gaussian states by squeezing the detection mode. The scaling with $N_{\theta}$ can be modified if $V_{\theta,[1,1]}^{-1}$ depends on $N_{\theta}$. For a fixed total energy a scaling $I_{\theta} \propto N_{\theta}^{3 / 2}$ can be achieved. This was proposed by Barnett, Fabre, and Maître (2003) for measuring a beam displacement. The quantum Cramér-Rao bound in Eq. (29) can be reached by homodyne detection with the local oscillator in this detection mode.

By using compact expressions of the quantum Fisher information for multimode Gaussian states, Šafránek and Fuentes (2016) developed a practical method to find optimal Gaussian probe states for the estimation of parameters encoded by Gaussian unitary channels. Applications of the method to the estimation of relevant parameters in singlemode and two-mode unitary channels, such as phase, singlemode squeezing, two-mode squeezing, and transmissivity of a beam splitter, confirmed that separable probes can achieve exactly the same precision as entangled probes, leading Šafránek and Fuentes (2016) to remark how entanglement does not play any significant role in achieving the Heisenberg limit for unitary Gaussian quantum metrology.

The same conclusion has been reached by considering the estimation of any small parameter $\theta$ encoded in Bogoliubov transformations, i.e., Gaussian unitary channels corresponding to arbitrary linear transformations of a set of $n$ canonical mode operators (Friis et al., 2015). In the limit of infinitesimal transformations $(\theta \ll 1)$, and considering an arbitrary (Gaussian or not) pure $n$-mode probe state with input mean photon number $N_{\theta}$, Friis et al. (2015) showed by means of a perturbative analysis that the maximal achievable quantum Fisher information scales as $I_{\theta} \propto N_{\theta}^{2}$, that is, at the Heisenberg limit. Remarkably, such a quantum-enhanced scaling requires nonclassical (e.g., squeezed) but not necessarily entangled states.

Further results on the use of bosonic probes and the role of mode entanglement in Gaussian and non-Gaussian quantum metrology are presented in Sec. III.B.

\section{G. Quantum channel discrimination}

A fundamental protocol which is closely related to quantum metrology is quantum channel discrimination (Childs, Preskill, and Renes, 2000; Acin, 2001; Sacchi, 2005; Lloyd, 2008; Tan et al., 2008; Invernizzi, Paris, and Pirandola, 2011; Pirandola, 2011), which may be seen as a sort of digitalized version of quantum metrology. Its basic formulation is binary and involves the task of distinguishing between two quantum channels $\mathcal{E}_{0}$ or $\mathcal{E}_{1}$ associated with two a priori probabilities $\pi_{0}:=\pi$ and $\pi_{1}=1-\pi$. During the 
encoding phase, one of such channels is picked by Alice and stored in a box which is then passed to Bob. In the decoding phase, Bob uses a suitable state at the input of the box and performs a quantum measurement of its output. Bob may also use ancillary systems which are quantum correlated with the input probes and are directly sent to the measurement. For the specific tasks of discriminating bosonic channels, the input is assumed to be constrained in energy, so that we fix the mean number of photons $\bar{n}$ per input probe, or, more strongly, the mean total number of photons which are globally irradiated through the box (Weedbrook et al., 2012).

Quantum channel discrimination is an open problem in general. However, when one fixes the input state, it is translated into an easier problem to solve, i.e., the quantum discrimination of the output states. In the binary case, this conditional problem has been fully solved by the so-called Helstrom bound which provides the minimum mean error probability $\bar{p}$ in the discrimination of any two states $\rho_{0}$ and $\rho_{1}$. Assuming equiprobable states $(\pi=1 / 2)$, this bound is simply given by their trace distance $D$, i.e., we have (Helstrom, 1976)

$$
\bar{p}=\frac{1}{2}\left[1-D\left(\rho_{0}, \rho_{1}\right)\right]
$$

In the case of multicopy discrimination, in which one probes the box $N$ times and one aims to distinguish the two outputs $\rho_{0}^{\otimes N}$ and $\rho_{1}^{\otimes N}$, the mean error probability $\bar{p}(N)$ may be not so easy to compute and, therefore, one resorts to suitable bounds. Using the quantum fidelity $F\left(\rho_{0}, \rho_{1}\right)$ from Eq. (11), and setting

$$
Q\left(\rho_{0}, \rho_{1}\right):=\inf _{0 \leq s \leq 1} \operatorname{Tr}\left(\rho_{0}^{s} \rho_{1}^{1-s}\right)
$$

one may then write (Fuchs and de Graaf, 1999; Audenaert et al., 2007; Banchi, Braunstein, and Pirandola, 2015)

$$
\frac{1-\sqrt{1-F\left(\rho_{0}, \rho_{1}\right)^{N}}}{2} \leq \bar{p}(N) \leq \frac{Q^{N}\left(\rho_{0}, \rho_{1}\right)}{2},
$$

where $Q^{N}\left(\rho_{0}, \rho_{1}\right) / 2$ is the quantum Chernoff bound (QCB) (Audenaert et al., 2007). In particular, the QCB is asymptotically tight for large $N$. Furthermore, it can be easily computed for arbitrary multimode Gaussian states (Pirandola and Lloyd, 2008).

Since the conditional output states can be optimally distinguished, the nontrivial part in quantum channel discrimination is the optimization of the mean error probability $\bar{p}$ over the input states. For this reason, it is an extremely rich problem and depending on the types of quantum channels, quantum correlations may play an important role or not. In the following, some specific cases are discussed in more detail.

Quantum channel discrimination has various practical applications. One which is very well known is quantum illumination (Lloyd, 2008; Tan et al., 2008) which forms the basis for a "quantum radar" (Barzanjeh et al., 2015). Despite the fact that entanglement is used at the input between the signal (sent to probe a potential target) and the idler (kept at the radar state for joint detection), entanglement is completely absent at the output between reflected and idler photons. Nevertheless the scheme assures a superior performance with respect to the use of coherent states; in particular, an increase by a factor of 4 of the exponent $-[\ln P(e)] / M$ of the asymptotic error probability $P(e)$ (where $M$ is the number of transmissions) (Tan et al., 2008). For this reason, the quantum illumination advantage has been studied in relation to the consumption of other discord-type quantum correlations beyond entanglement (Bradshaw et al., 2016; Weedbrook et al., 2016). More precisely, the enhanced performance of quantum illumination (with respect to signal probing not assisted by an idler) may be connected to the amount of discord which is expended to resolve the target (i.e., to encode the information about its presence or absence). Quantum illumination was demonstrated experimentally by Lopaeva et al. (2013) and Zhang et al. (2013, 2015).

Another application of quantum channel discrimination is quantum reading (Pirandola, 2011). Here the basic aim is to discriminate between two different channels which are used to encode an information bit in a cell of a classical memory. In an optical setting, this means to discriminate between two different reflectivities, generally assuming the presence of decoherence effects, such as background stray photons. The maximum amount of bits per cell that can be read is called "quantum reading capacity" (Pirandola et al., 2011). This model has also been studied in the presence of thermal and correlated decoherence, as that arising from optical diffraction (Lupo et al., 2013). In all cases, the classical benchmark associated with coherent states can be largely beaten by nonclassical states, as long as the mean number of photons hitting the memory cells is suitably low.

Depending on the regime, one may choose a different type of nonclassical states. In the presence of thermal decoherence induced by background photon scattering, two-mode squeezed vacuum states between signal modes (reading the cells) and idler modes (kept for detection) are nearly optimal. However, in the absence of decoherence, the sequential readout of an ideal memory (where one of the reflectivities is exactly $100 \%$ ) is optimized by number states at the input (Nair, 2011). Roga, Buono, and Illuminati (2015) showed that, in specific regimes, the quantum advantage can be related to a particular type of quantum correlations, the discord of response, which is defined as the trace, or Hellinger, or Bures minimum distance from the set of unitarily perturbed states (Roga, Giampaolo, and Illuminati, 2014). Roga, Buono, and Illuminati (2015) also identified particular regimes in which strongly discordant states are able to outperform pure entangled transmitters.

Consider the specific case of unitary channel discrimination. Suppose that the task is to decide whether a unitary $U_{\theta}$ was applied or not to a probing subsystem $A$ of a joint system $(A, B)$. In other words, the aim is to discriminate between the two possible output states $\rho_{\theta}=\left(U_{\theta} \otimes \mathbb{I}\right) \rho\left(U_{\theta} \otimes \mathbb{I}\right)^{\dagger}$ (when the unitary $U_{\theta}$ has acted on $A$ ) or $\rho$ (equal to the input, when the identity has acted on $A$ instead). In the limit of an asymptotically large number $N \gg 1$ of copies of $\rho$, the minimal probability of error in distinguishing between $\rho_{\theta}$ and $\rho$, using an optimal discrimination strategy scales approximately as the QCB $Q\left(\rho, \rho_{\theta}\right)^{N} / 2$.

It is clear that the quantity $1-Q\left(\rho, \rho_{\theta}\right)$ plays a similar role in the present discrimination context as the quantum Fisher 
information in the parameter estimation scheme. One can therefore introduce an analogous figure of merit quantifying the worst case ability to discriminate, guaranteed by the state $\rho$. The discriminating strength of the bipartite state $\rho$ with respect to the probing system $A$ is then defined as (Farace et al., 2014)

$$
D^{(A)}(\rho)=\min _{H}\left[1-Q\left(\rho, \rho_{\theta}\right)\right]
$$

where the minimization is performed once more over all generators $H$ within a given nondegenerate spectrum.

As proven by Farace et al. (2014), the discriminating strength is another measure of discord-type correlations in the input state $\rho$, which vanishes if and only if $\rho$ is a classicalquantum state as in Eq. (20). The discriminating strength is also computable in closed form for all finite-dimensional states such that subsystem $A$ is a qubit. In the latter case, the discriminating strength turns out to be proportional to the local quantum uncertainty (Girolami, Tufarelli, and Adesso, 2013), a further measure of discord-type correlations defined as in Eq. (22), but with the quantum Fisher information replaced by the Wigner-Yanase skew information (Girolami, Tufarelli, and Adesso, 2013). The discriminating strength has also been extended to continuous-variable systems and evaluated for special families of two-mode Gaussian states restricting the minimization in Eq. (33) to Gaussianity-preserving generators (i.e., quadratic Hamiltonians) (Rigovacca et al., 2015).

Finally, note that the presence and use of quantum correlations beyond entanglement has also been investigated in other tasks related to metrology and illumination, such as ghost imaging with (unentangled) thermal source beams (Ragy and Adesso, 2012). Adopting a coarse-grained twomode description of the beams, quantum discord was found to be relevant for the implementation of ghost imaging in the regime of low illumination, while more generally total correlations in the thermal source beams were shown to determine the quality of the imaging, as quantified by the signal-to-noise ratio.

\section{H. Average precision in black-box settings}

The results reviewed so far in this section highlight a clear resource role for quantum discord, specifically measured by operational quantifiers such as the interferometric power and the discriminating strength, in black-box metrology settings, elucidating, in particular, how quantum correlations beyond entanglement manifest themselves as coherence in all local bases for the probing subsystem. Discordant states, i.e., all states but those of Eq. (20), are not only disturbed by all possible local measurements on $A$, but are also modified byhence sensitive to-all nontrivial unitary evolutions on subsystem $A$. This is exactly the ingredient needed for the estimation and discrimination tasks.

In practice, however, one might want to assess the general purpose performance of probe states, rather than their worst case scenario only. One can then introduce alternative figures of merit quantifying how suitable a state is, on average, for estimation or discrimination of unitary transformations, when the average is performed over all generators of a fixed spectral class. This can be done by replacing the minimum with an average according to the Haar measure, in Eqs. (22) and (33), respectively. Such a study has been carried out by Farace et al. (2016) by defining the local average Wigner-Yanase skew information, which corresponds to the average version of the discriminating strength in case the probing subsystem $A$ is a qubit (Farace et al., 2014).

Unlike the minimum, the average skew information is found to not be a measure of discord anymore. In particular, it vanishes only on states of the form $\mathbb{I}^{A} / d_{A} \otimes \tau^{B}$, that is, tensor product states between a maximally mixed state on $A$, and an arbitrary state on $B$ (Farace et al., 2016). This entails that, to ensure a reliable discrimination of local unitaries on average, the input states need to have either one of these two (typically competing) ingredients: nonzero local purity of the probing subsystem, or nonzero correlations (of any nature) with the ancilla. The interplay between the average performance and the minimum one, which instead relies on discord, as well as a study of the role of entanglement, is detailed by Farace et al. (2016). A similar study was recently performed in continuous-variable systems, in which the average quantum Fisher information for estimating the amount of squeezing applied to an input single-mode probe, without previous knowledge on the phase of the applied squeezing, was investigated with and without the use of a correlated ancilla (Rigovacca et al., 2017).

\section{IDENTICAL PARTICLES}

Measuring devices and sensors operating with many-body systems are among the most promising instances for which quantum-enhanced measurements can be actually experimented; indeed, their large numbers of elementary constituents play the role of resources according to which the accuracy of parameter estimation can be scaled. Typical instances in which the quantum-enhanced measurement paradigm has been studied are in fact interferometers based on ultracold atoms confined in optical lattices (Leggett, 2001, 2006, Pitaevskii and Stringari, 2003; Pethick and Smith, 2004; Gerry and Knight, 2005; Haroche and Raimond, 2006; Inguscio, 2006; Köhl and Esslinger, 2006; Giorgini, Pitaevskii, and Stringari, 2008; Cronin, Schmiedmayer, and Pritchard, 2009); Yukalov, 2009), where a precise control on the state preparation and the dynamics can nowadays be obtained. These systems are made of spatially confined bosons or fermions, i.e., of constituents behaving as identical particles, a fact that has not been properly taken into account in most of the literature.

In systems of distinguishable particles, the notion of separability and entanglement is well established (Horodecki et al., 2009): it is strictly associated with the natural tensor product structure of the multiparticle Hilbert space and expresses the fact that one is able to identify each one of the constituent subsystems with their corresponding single-particle Hilbert spaces. On the contrary, in order to describe identical particles one must extract from the tensor product structure of the whole Hilbert space either the symmetric (bosonic) or the antisymmetric (fermionic) sector (Feynman, 1994; Sakurai, 1994). This fact demands a more general approach to the notions of nonlocality and 
entanglement based not on the particle aspect proper for first quantization, but rather on the mode description typical of second quantization (Summers and Werner, 1985, 1987a, 1987b; Narnhofer, 2003; Barnum et al., 2004, 2005; Zanardi, Lidar, and Lloyd, 2004; Benatti, Floreanini, and Marzolino, 2010a, 2011, 2012a, 2012b, 2014; Argentieri et al., 2011; Marzolino, 2013; Benatti and Floreanini, 2014; Benatti, Floreanini, and Titimbo, 2014; Benatti et al., 2017).

The notion of entanglement in many-body systems was already addressed and discussed in the literature: for instance, see Li et al. (2001), Paskauskas and You (2001), Schliemann et al. (2001), Eckert et al. (2002), Ghirardi, Marinatto, and Weber (2002), Hines, McKenzie, and Milburn (2003), Micheli et al. (2003), Wiseman and Vaccaro (2003), Schuch, Verstraete, and Cirac (2004), Shi (2004), Dowling, Doherty, and Wiseman (2006), Banuls, Cirac, and Wolf (2007), Lewenstein et al. (2007), Amico et al. (2008), Bloch, Dalibard, and Zwerger (2008), Kraus et al. (2009), Grabowski, Kuś, and Marmo (2011), Calabrese, Mintchev, and Vicari (2012), Modi et al. (2012), Song et al. (2012), Balachandran et al. (2013), and Tichy et al. (2013) and references therein. Nevertheless, only limited results actually apply to the case of identical particles and their applications to quantum-enhanced measurements. From the existing literature on possible metrological uses of identical particles, there emerges as a controversial issue the distinction between particle and mode entanglement. Before illustrating the general approach developed by Benatti, Floreanini, and Marzolino (2010a, 2012a, 2014) within which this matter can be settled, we shortly overview the main aspects of the problem. Readers who feel that the discussion whether the states in question are to be considered as entangled or not is rather academic may be reassured by the very pragmatical result that independently of this discussion, systems of indistinguishable bosons offer a metrological advantage over distinguishable particles, in the sense that for certain measurements one would have to massively entangle the latter for obtaining the same sensitivity as one obtains "for free" from the symmetrized states of the former. This we show explicitly in Sec. III.B.

Entanglement based on the particle description proper for first quantization was discussed for pure states by Li et al. (2001), Paskauskas and You (2001), and Schliemann et al. (2001). In the fermionic case, Slater determinants are identified as the only nonentangled fermionic pure states, for, due to the Pauli exclusion principle, they are the least correlated many-particle states. In the case of bosons, two inequivalent notions of particle entanglement have been put forward: Paskauskas and You (2001) declared a pure bosonic state is nonentangled if and only if all particles are prepared in the same single-particle state, thus leading to a bosonic product state. Li et al. (2001) identified bosonic nonentangled pure states as those corresponding to permanents (the bosonic analog of Slater determinants); in other words, a pure bosonic state is nonentangled not only when, as in the first approach mentioned, it is a product state, but also when all bosons are prepared in pairwise orthogonal single-particle states. The particle-based entanglement was then studied by Eckert et al. (2002) for both bosons and fermions, and then generalized within a more mathematical and abstract setting in Grabowski, Kuś, and Marmo (2011, 2012).

From the point of view of particle description, a different perspective was provided by Ghirardi, Marinatto, and Weber (2002, 2004), based on the fact that nonentangled pure states should possess a complete set of local properties, identifiable by local measurements. For a more recent nonstandard approach, still based on the particle description, see Lo Franco and Compagno (2016).

In all these approaches, only bipartite systems consisting of two identical particles are discussed; however, in the fermionic case, simple necessary and sufficient criteria of many particle entanglement, based on the single-particle reduced density matrix, have been elaborated by Plastino, Manzano, and Dehesa (2009).

A change of perspective occurred in Vedral (2003), Barnum et al. (2004, 2005), and Zanardi, Lidar, and Lloyd (2004) where the focus moved from particles to orthonormal modes; within this approach, states that are not mode entangled are characterized by correlations that can be explained in terms of joint classical occupation probabilities of the modes. It then follows that entanglement and nonlocality depend on the mode description which has been chosen. In all cases, however, identical-particle states represented by fermionic Slater determinants or bosonic permanents are neither particle nor mode entangled in direct conflict with claims that these states are particle entangled and hence metrologically useful (Demkowicz-Dobrzański, Jarzyna, and Kołodiński, 2015). In an attempt to resolve the conflict, Killoran, Cramer, and Plenio (2014) showed that such a pseudo or "fluffy-bunny" entanglement as called in Wiseman, Bartlett, and Vaccaro (2004) and Beenakker (2006), which is due to bosonic state symmetrization, can be turned into the entanglement of distinguishable, and thus metrologically accessible, modes; however, the operations needed for such a transformation are nonlocal in the mode picture and thus ultimately responsible for the achieved sub-shot-noise accuracies.

The variety of approaches regarding entanglement in identical-particle systems and their use in metrological applications, in particular, whether entanglement is necessary or not to achieve sub-shot-noise accuracies, can be looked at from the unifying point of view provided by the algebraic approach to quantum many-body systems proper to second quantization (Strocchi, 1985; Bratteli and Robinson, 1987). Within this scheme, we first address the relations between mode and particle entanglement. Then we focus on specific quantum metrological issues and show that neither entangled states nor preliminary state preparation such as spin squeezing is necessary in order to achieve sub-shot-noise accuracies using systems of identical particles.

\section{A. Particle and mode entanglement}

In quantum mechanics, indistinguishable particles cannot be identified by specific labels, whence their states must be either completely symmetrized (bosons) or antisymmetrized (fermions). This makes second quantization a most suited approach to deal with them, while the particle representation is proper for first quantization results to be too restrictive for a consistent treatment of entangled identical particles. 
As a simple example of the consequences of indistinguishability, consider two qubits, each of them described by the Hilbert space $\mathcal{H}=\mathbb{C}^{2}$, where we select the orthonormal basis $\{|0\rangle,|1\rangle\}$ of eigenvectors of $\sigma_{z}: \sigma_{z}|0\rangle=|0\rangle, \sigma_{z}|1\rangle=-|1\rangle$. If the two qubits describe distinguishable particles, the tensor product structure of the common Hilbert space $\mathbb{C}^{4}=\mathbb{C}^{2} \otimes \mathbb{C}^{2}$ exhibits the fact that one knows which is qubit 1 and which one is qubit 2. Instead, if the two qubits are indistinguishable, the corresponding Hilbert space loses its tensor product structure and becomes, in the bosonic case, the three-dimensional subspace $\mathbb{C}^{3}$ spanned by the orthogonal two-particle symmetric vectors $|00\rangle,|11\rangle$, and $\left|\psi_{+}^{(2)}\right\rangle:=(|01\rangle+|10\rangle) / \sqrt{2}$, whereas in the fermionic case it reduces to the antisymmetric two-particle vector $\left|\psi_{-}^{(2)}\right\rangle:=(|01\rangle-|10\rangle) / \sqrt{2}$.

An important consequence of this fact is that generic mixed states of indistinguishable particles must be represented by density matrices that arise from convex linear combinations of projectors onto symmetrized or antisymmetrized pure states. Indeed, while operators must be symmetrized in order to comply with particle indistinguishability, by symmetrizing density matrices of distinguishable particles, say sending $\rho=$ $\rho_{1} \otimes \rho_{2}$ into $\left(\rho_{1} \otimes \rho_{2}+\rho_{2} \otimes \rho_{1}\right) / 2$ one cannot in general obtain an appropriate bosonic or fermionic state, unlike sometimes stated in the literature (Demkowicz-Dobrzański, Jarzyna, and Kołodiński, 2015). For instance, the two qubit symmetric mixed state $\rho \otimes \rho$ cannot be a fermionic density matrix since the only fermionic state is $\left|\psi_{-}^{(2)}\right\rangle\left\langle\psi_{-}^{(2)}\right|$. On the other hand, in the bosonic case, $\rho \otimes \rho$ can be a bona fide bosonic state only if $\rho$ is pure. Indeed, as $\rho \otimes \rho\left|\psi_{-}^{(2)}\right\rangle=$ $\operatorname{det}(\rho)\left|\psi_{-}^{(2)}\right\rangle$, if the determinant of the density matrix $\operatorname{det}(\rho) \neq 0$, then $\rho \otimes \rho$ has support also in the antisymmetric component of the Hilbert space. A careful discussion of the relationship between permutationally invariant density matrices and symmetric and antisymmetric states can be found in Damanet, Braun, and Martin (2016).

Instead of the standard particle picture, second quantization is based on the so-called mode picture. In general, a mode is any of the normalized vectors $|\psi\rangle$ of the same single-particle Hilbert space $\mathcal{H}$ that is used to describe each one of a system of identical particles. In practice, one fixes an orthonormal basis $\left\{\left|\psi_{i}\right\rangle\right\}$ in $\mathcal{H}$ and populates the $i$ th mode by acting on the so-called vacuum vector with powers of the creation operator $a_{i}^{\dagger}, a_{i}^{\dagger}|0\rangle=\left|\psi_{i}\right\rangle$, while the adjoint operators $a_{i}$ annihilate the vacuum $a_{i}|0\rangle=0$. For bosons, $\left[a_{i}, a_{j}^{\dagger}\right]=a_{i} a_{j}^{\dagger}-a_{j}^{\dagger} a_{i}=\delta_{i j}$ and one can find arbitrarily many particles in a given mode, while for fermions $\left\{a_{i}, a_{j}^{\dagger}\right\}=a_{i} a_{j}^{\dagger}+a_{j}^{\dagger} a_{i}=\delta_{i j}$ and each mode can be occupied by one fermion at most.

Typical modes are given by the eigenvectors of a given single-particle Hamiltonian, orthogonal polarization directions, the left and right positions in a double-well potential, or the atomic positions in an optical lattice. In the case of free photons, typical modes are plane waves labeled by wave vector and polarization, arising from the quantization of classical electrodynamics in terms of independent harmonic oscillators. Within this picture, saying that there are $n$ photons in a certain energy mode can also be interpreted as a quantum oscillator being promoted to its $n$th excited state.
In dealing with distinguishable particles, quantum entanglement (Horodecki et al., 2009) is basically approached by referring to the tensor product structure of the total Hilbert space which embodies the fact that particles can be identified. From the previous discussion, it follows that, in the case of identical particles, one ought to consider the entanglement of modes rather than the entanglement of particles. The relations between the two approaches have been studied in detail by Benatti, Floreanini, and Titimbo (2014); here we briefly compare them in the case of two two-mode indistinguishable bosons associated with orthogonal single-particle pure states $\left|\psi_{1,2}\right\rangle \in \mathcal{H}$, as, for instance, orthogonal polarization states, described by creation and annihilation operators $a_{i}, a_{i}^{\dagger}$, $i=1,2$. The pure state

$$
|1,1\rangle=a_{1}^{\dagger} a_{2}^{\dagger}|0\rangle
$$

belongs to the two-particle sector of the symmetric Fock space and represents a pure state with one boson in each mode. In the particle picture of first quantization, the same state corresponds to the symmetrized vector

$$
\left|\psi_{+}\right\rangle=\frac{1}{\sqrt{2}}\left(\left|\psi_{1}\right\rangle \otimes\left|\psi_{2}\right\rangle+\left|\psi_{2}\right\rangle \otimes\left|\psi_{1}\right\rangle\right)
$$

which describes a balanced superposition of two states: one with the first particle in the state $\left|\psi_{1}\right\rangle$ and the second particle in the state $\left|\psi_{2}\right\rangle$, the other with the two states exchanged. The net result is that, given the state (35), we can say only that one particle is surely in the state $\left|\psi_{1}\right\rangle$ and that the other one is surely in the state $\left|\psi_{2}\right\rangle$, but we cannot attribute a specific state to a specific particle.

As an example of the misunderstandings that can result from sticking to the particle picture when dealing with identical particles, state (35) is often referred to as entangled: actually, it is only formally so in the particle picture, while, as we shall show, it is separable in the mode picture. Indeed, Eq. (35) would clearly display particle entanglement as the state cannot be written as a tensor product of single-particle states. Instead, in Eq. (34), the same state is expressed as the action on the vacuum state of two independent creation operators and thus should correspond to a mode-separable state under whichever a meaningful definition of mode entanglement one should adopt. We later give solid ground to this latter statement, but first we show that, while for distinguishable particles the state (35) is the prototype of an entangled pure state, it is nevertheless separable in the sense specified next.

\section{Particle entanglement}

For distinguishable particles, bipartite observables are termed local if they are tensor products $O_{12}=O_{1} \otimes O_{2}$ of single-particle observables $O_{1} \otimes \mathbb{I}$ pertaining to particle 1 and $\mathbb{I} \otimes \mathrm{O}_{2}$ pertaining to particle 2 , where $\mathbb{I}$ denotes the identity operator, namely, if they address each particle independently. Consequently, in such a context, locality is associated with the addressability of single particles and referred to as particle locality in the following. Then a bipartite pure state $\left|\psi_{12}\right\rangle$ is 
separable if and only if the expectation values $\left\langle O_{12}\right\rangle_{12}=$ $\left\langle\psi_{12}\left|O_{1} \otimes O_{2}\right| \psi_{12}\right\rangle$ of all local observables factorize

$$
\left\langle O_{12}\right\rangle_{12}=\left\langle O_{1} \otimes \mathbb{I}\right\rangle_{12}\left\langle\mathbb{I} \otimes O_{2}\right\rangle_{12} .
$$

Indeed, if $\left|\psi_{12}\right\rangle=\left|\psi_{1}\right\rangle \otimes\left|\psi_{2}\right\rangle$ the equality (36) clearly holds. On the other hand, if the equality holds, by using the Schmidt decomposition of $\left|\psi_{12}\right\rangle=\sum_{i} \lambda_{i}\left|\phi_{i}^{(1)}\right\rangle \otimes\left|\phi_{i}^{(2)}\right\rangle$ and choosing $O_{1}=\left|\phi_{i}^{(1)}\right\rangle\left\langle\phi_{i}^{(1)}\left|, O_{2}=\right| \phi_{j}^{(2)}\right\rangle\left\langle\phi_{j}^{(2)}\right|$ one finds that only one Schmidt coefficient can be different from zero. Instead, for distinguishable particles, the state $\left|\psi_{+}\right\rangle$in Eq. (35) violates the equality for $O_{1}=\left|\psi_{1}\right\rangle\left\langle\psi_{1}\right|$ and $O_{2}=\left|\psi_{2}\right\rangle\left\langle\psi_{2}\right|$ and is thus entangled.

If the two particles are identical, they cannot be addressed individually; one has thus to refer to observables that specify properties attributable to single particles without specifying to which one of them. It therefore follows that, in the case of two identical particles, appropriate single-particle observables cannot be of the form $O \otimes \mathbb{I}$ or $\mathbb{I} \otimes O$, but of the symmetrized form

$$
O \otimes \mathbb{I}+\mathbb{I} \otimes O
$$

Consider the single-particle property described by the onedimensional projector $|\psi\rangle\langle\psi|$; for two indistinguishable particles the property "one particle is in the state $|\psi\rangle$," must then be represented by the symmetric projection (Ghirardi, Marinatto, and Weber, 2002, 2004)

$$
\begin{aligned}
\mathcal{E}_{\psi}= & |\psi\rangle\langle\psi|\otimes(\mathbb{I}-|\psi\rangle\langle\psi|)+(\mathbb{I}-|\psi\rangle\langle\psi|) \otimes| \psi\rangle\langle\psi| \\
& +|\psi\rangle\langle\psi|\otimes| \psi\rangle\langle\psi| .
\end{aligned}
$$

Similarly, the projector corresponding to the two qubits possessing two different properties $\left|\psi_{1,2}\right\rangle\left\langle\psi_{1,2}\right|$ must be $P_{12}^{\text {symm }}=\left|\psi_{1}\right\rangle\left\langle\psi_{1}|\otimes| \psi_{2}\right\rangle\left\langle\psi_{2}|+| \psi_{2}\right\rangle\left\langle\psi_{2}|\otimes| \psi_{1}\right\rangle\left\langle\psi_{1}\right| . \quad$ If $\left\langle\psi_{1} \mid \psi_{2}\right\rangle=0$, then $P_{12}^{\text {symm }}=\mathcal{E}_{\psi_{1}} \mathcal{E}_{\psi_{2}}$. It then follows that, despite the formal entanglement of $\left|\psi_{+}\right\rangle$, the factorization of mean values as in Eq. (36) still holds; indeed,

$\left\langle\psi_{+}\left|\mathcal{E}_{\psi_{1}}\right| \psi_{+}\right\rangle=\left\langle\psi_{+}\left|\mathcal{E}_{\psi_{2}}\right| \psi_{+}\right\rangle=\left\langle\psi_{+}\left|P_{12}^{\mathrm{sym}}\right| \psi_{+}\right\rangle=1$.

Therefore, from the particle point of view, namely, of attributable properties, the formally entangled state $\left|\psi_{+}\right\rangle$is indeed separable.

\section{Mode entanglement}

Based on the attribution of properties to identical particles, the previous discussion is developed in first quantization terms, namely, using symmetrized states and observables. From the point of view of second quantization, separability and entanglement are instead to be related to the algebraic structure of Bose and Fermi systems rather than to the possibility of attributing individual properties: this is the point of view usually adopted in the analysis of many-body systems, for which the primary object of investigation is the algebras of operators rather than their representations on particular Hilbert spaces (Emch, 1972; Strocchi, 1985, 2008a, 2008b, 2012Bratteli and Robinson, 1987; Haag, 1992; Thirring, 2002).
In order to appropriately formulate the notion of entanglement and nonlocality in systems made of identical particles, the leading intuition is that there is no a priori given tensor product structure, reminiscent of particle identification, neither in the Hilbert space nor in the algebra of observables. Therefore, questions about entanglement and separability are meaningful only with reference to specific classes of observables. Within this broader context, entanglement becomes a caption for nonlocal quantum correlations between observables exhibited by certain quantum states [the original discussion can be found in Summers and Werner (1985, 1987a, 1987b); further developments can be found in Halvorson and Clifton (2000), Clifton and Halvorson (2001), Keyl, Schlingemann, and Werner (2003), Verch and Werner (2005), Keyl et al. (2006), and Moriya (2006)].

Polynomials in creation and annihilation operators can be used to generate bosonic and fermionic algebras $\mathcal{A}$ containing all physically relevant many-body observables. Quite in general, physical states are given by positive and normalized linear functionals $\omega: \mathcal{A} \rightarrow \mathbb{C}$ associating with any operator $A \in \mathcal{A}$ its mean value $\omega(A)$, such that positive observables $A \geq 0$ are mapped to positive numbers $\omega(A) \geq 0$ and $\omega(\mathbb{I})=1$. Typical instances of states are the standard expectations obtained by tracing with respect to a given density matrix $\rho, \omega(A)=\operatorname{Tr}(\rho A)$; note, however, that in the presence of infinitely many degrees of freedom, not all physically meaningful states, such as, for instance, the thermal ones, can be represented by density matrices (Narnhofer, 2003).

Within such an algebraic approach, one can study the entanglement between observables of $\mathcal{A}$ with respect to a state $\omega$ by considering algebraic bipartitions (Benatti, Floreanini, and Marzolino, 2010a, 2014), namely, any couple of subalgebras $\left(\mathcal{A}_{1}, \mathcal{A}_{2}\right)$ of $\mathcal{A}$, having only the identity in common $\mathcal{A}_{1} \cap \mathcal{A}_{2}=\mathbb{I}$, such that the linear span of products of their operators generates the entire algebra $\mathcal{A}$ and

(1) $\left[A_{1}, A_{2}\right]=0$ for all $A_{1} \in \mathcal{A}_{1}$ and $A_{2} \in \mathcal{A}_{2}$ in the bosonic case;

(2) $\left[A_{1}^{e}, A_{2}\right]=0$ for all even elements $A_{1}^{e} \in \mathcal{A}_{1}^{e}$ and for all $A_{2} \in \mathcal{A}_{2}$, and, similarly, $\left[A_{1}, A_{2}^{e}\right]=0$ for all even elements $A_{2}^{e} \in \mathcal{A}_{2}^{e}$ and for all $A_{1} \in \mathcal{A}_{1}$, in the fermionic case,

where the even elements $A_{1,2}^{e} \in \mathcal{A}_{1,2}$ are those remaining invariant under the transformation of creation and annihilation operators $\vartheta\left(a_{i}^{\dagger}\right)=-a_{i}^{\dagger}$ and similarly for $a_{i}$. They generate the so-called even subalgebras $\mathcal{A}_{1,2}^{e} \subset \mathcal{A}$ which contain the only fermionic operators accessible to experiments.

These desiderata embody the notion of algebraic independence and generalize the tensor product structure typical of the particle picture. Within this more general context, an operator $\mathcal{O}$ is called $\left(\mathcal{A}_{1}, \mathcal{A}_{2}\right)$ local if of the form $\mathcal{O}=A_{1} A_{2}$, $A_{1} \in \mathcal{A}_{1}$, and $A_{2} \in \mathcal{A}_{2}$. Furthermore, a state $\omega$ is called $\left(\mathcal{A}_{1}, \mathcal{A}_{2}\right)$ separable if the expectation values of all $\left(\mathcal{A}_{1}, \mathcal{A}_{2}\right)$ local operators can be decomposed into convex combinations of expectations:

$$
\omega\left(A_{1} A_{2}\right)=\sum_{i} \lambda_{k} \omega_{k}^{(1)}\left(A_{1}\right) \omega_{k}^{(2)}\left(A_{2}\right)
$$


in terms of other states $\omega_{i}^{(1)}, \omega_{i}^{(2)}$ with $\lambda_{k}>0, \sum_{i} \lambda_{k}=1$; otherwise, $\omega$ is called $\left(\mathcal{A}_{1}, \mathcal{A}_{2}\right)$ entangled. ${ }^{2}$

In the standard case of bipartite entanglement for pairs of distinguishable particles (Horodecki et al., 2009), entangled states are all density matrices $\rho$ which cannot be written in the form

$$
\rho=\sum_{k} \lambda_{k} \rho_{k}^{(1)} \otimes \rho_{k}^{(2)}, \quad \lambda_{k} \geq 0, \quad \sum_{k} \lambda_{k}=1,
$$

with $\rho_{k}^{(1)}$ and $\rho_{k}^{(2)}$ density matrices of the two parties. That the algebraic definition reduces to the standard one becomes apparent in the case of two qubits by choosing the algebraic bipartition $\mathcal{A}_{1}=\mathcal{A}_{2}=\mathcal{M}_{2}$, where $\mathcal{M}_{2}$ is the algebra of $2 \times 2$ complex matrices and the expectation value $\omega\left(A_{1} A_{2}\right)=$ $\operatorname{Tr}\left(\rho A_{1} \otimes A_{2}\right)$.

Henceforth, we shall focus on many-body systems, whose elementary constituents can be found in $\mathfrak{M}$ different states or modes described by a discrete set of annihilation and creation operators $\left\{a_{i}, a_{i}^{\dagger}\right\}_{i \in I}$; this is a very general framework, useful for the description of physical systems in quantum optics, in atomic, and in condensed matter physics. A bipartition of the $\mathfrak{M}$-mode algebra $\mathcal{A}$ associated with the system can then be easily obtained by considering two disjoint sets $\left\{a_{i}, a_{i}^{\dagger} \mid i=\right.$ $1,2, \ldots, m\}$ and $\left\{a_{j}, a_{j}^{\dagger} \mid j=m+1, m+2, \ldots, \mathfrak{M}\right\}, \mathfrak{M}$ being possibly infinite. The two sets form subalgebras $\mathcal{A}_{1,2}$ that indeed constitute an algebraic bipartition of $\mathcal{A}$; in practice, it is determined by the integer $0<m<\mathfrak{M}$.

As for distinguishable particles, the case of pure states is easier. Pure states in the algebraic context ${ }^{3}$ correspond to those expectations on $\mathcal{A}$ that cannot be written as convex combinations of other expectations. For them the separability condition (40) simplifies: one can indeed prove (Benatti and Floreanini, 2016) that pure states $\omega$ on $\mathcal{A}$ are separable with respect to a given bipartition $\left(\mathcal{A}_{1}, \mathcal{A}_{2}\right)$ if and only if

$$
\omega\left(A_{1} A_{2}\right)=\omega\left(A_{1}\right) \omega\left(A_{2}\right),
$$

for all local operators $A_{1} A_{2}$. In other terms, separable pure states are just product states satisfying the factorization property (36) that holds for pure states of bipartite systems of distinguishable particles.

\section{B. Mode entanglement and metrology: Bosons}

The differences between mode entanglement and standard entanglement are best appreciated in the case of $N$ bosons that can occupy $\mathfrak{M}$ different modes; this is a very general situation encountered, for instance, in ultracold gases consisting of bosonic atoms confined in a multiple site optical lattices.

\footnotetext{
${ }^{2}$ This generalized definition of separability can be easily extended to the case of more than two partitions; for instance, in the case of an $n$ partition, Eq. (40) would extend to $\omega\left(A_{1} A_{2} \cdots A_{n}\right)=$ $\sum_{k} \lambda_{k} \omega_{k}^{(1)}\left(A_{1}\right) \omega_{k}^{(2)}\left(A_{2}\right) \cdots \omega_{k}^{(n)}\left(A_{n}\right)$.

${ }^{3}$ In the algebraic description, Hilbert spaces are a by-product of the algebraic structure and of the expectation functional (state) defined on it (Bratteli and Robinson, 1987).
}

These systems turn out to be a unique laboratory for studying quantum effects in many-body physics, e.g., in quantum phase transition and matter interference phenomena, and also for applications in quantum information (Pitaevskii and Stringari, 2003; Pethick and Smith, 2004; Gerry and Knight, 2005; Haroche and Raimond, 2006; Inguscio, 2006; Köhl and Esslinger, 2006; Leggett, 2006; Giorgini, Pitaevskii, and Stringari, 2008; Cronin, Schmiedmayer, and Pritchard, 2009; Yukalov, 2009).

The algebra $\mathcal{A}$ is in this case generated by creation and annihilation operators $a_{i}^{\dagger}, a_{i}, i=1,2, \ldots, \mathfrak{M}$, obeying the commutation relations $\left[a_{i}, a_{j}^{\dagger}\right]=\delta_{i j}$. The reference state $\omega$ is given by the expectations with respect to the vacuum state $|0\rangle$, $a_{i}|0\rangle=0$ for all $1 \leq i \leq \mathfrak{M}$, so that the natural states whose entanglement properties need be studied are vectors in the Fock Hilbert space $\mathcal{H}$ spanned by the many-body Fock states

$$
\begin{aligned}
& \left|n_{1}, n_{2}, \ldots, n_{\mathfrak{M}}\right\rangle \\
& \quad=\frac{1}{\sqrt{n_{1} ! n_{2} ! \cdots n_{\mathfrak{M}} !}}\left(a_{1}^{\dagger}\right)^{n_{1}}\left(a_{2}^{\dagger}\right)^{n_{2}} \cdots\left(a_{M}^{\dagger}\right)^{n_{\mathfrak{M}}}|0\rangle,
\end{aligned}
$$

or density matrices acting on it.

Given a bipartition $\left(\mathcal{A}_{1}, \mathcal{A}_{2}\right)$ defined by two disjoint groups of creation and annihilation operators, any element $A_{1} \in \mathcal{A}_{1}$ commutes with any element $A_{2} \in \mathcal{A}_{2}$, i.e., $\left[\mathcal{A}_{1}, \mathcal{A}_{2}\right]=0$.

In this framework, a necessary and sufficient condition for pure states $|\psi\rangle$ to be separable with respect to a given bipartition $\left(\mathcal{A}_{1}, \mathcal{A}_{2}\right)$ is that they be generated by acting on the vacuum state with $\left(\mathcal{A}_{1}, \mathcal{A}_{2}\right)$ local operators (Benatti, Floreanini, and Marzolino, 2012a),

$$
|\psi\rangle=\mathcal{P}\left(a_{1}^{\dagger}, \ldots, a_{m}^{\dagger}\right) \cdot \mathcal{Q}\left(a_{m+1}^{\dagger}, \ldots, a_{\mathfrak{M}}^{\dagger}\right)|0\rangle,
$$

where $\mathcal{P}$ and $\mathcal{Q}$ are polynomials in the creation operators relative to the first $m$ modes and the last $\mathfrak{M}-m$ modes, respectively. Pure states that cannot be cast in this form are thus $\left(\mathcal{A}_{1}, \mathcal{A}_{2}\right)$ entangled.

When the state of the bosonic many-body system is not pure, it can be described by a density matrix $\rho$ in general not diagonal with respect to the Fock basis (43); since density matrices form a convex set whose extremal points are projectors onto pure states, one deduces that generic mixed states $\rho$ can be $\left(\mathcal{A}_{1}, \mathcal{A}_{2}\right)$ separable if and only if they are convex combinations of $\left(\mathcal{A}_{1}, \mathcal{A}_{2}\right)$ separable one-dimensional projections.

An interesting application of these general considerations is given by a system of $N$ bosons that can be found in just two modes $\mathfrak{M}=2$. In the Bose-Hubbard approximation, $N$ ultracold bosonic atoms confined in a double-well potential can be effectively described in this way. The two creation operators $a_{1}^{\dagger}$ and $a_{2}^{\dagger}$ generate out of the vacuum bosons in the two wells, so that the Fock basis (43) can be conveniently relabeled in terms of the integer $k$ counting the number of bosons in the first well:

$$
|k, N-k\rangle=\frac{\left(a_{1}^{\dagger}\right)^{k}\left(a_{2}^{\dagger}\right)^{N-k}}{\sqrt{k !(N-k) !}}|0\rangle, \quad 0 \leq k \leq N .
$$

Furthermore, $a_{1}, a_{1}^{\dagger}$, respectively, $a_{2}, a_{2}^{\dagger}$, generate two commuting subalgebras $\mathcal{A}_{1}$ and $\mathcal{A}_{2}$ that together in turn 
generate the whole algebra $\mathcal{A}$; it is the simplest bipartition of the system one can obtain by means of the operators $a_{1}, a_{1}^{\dagger}$ and $a_{2}, a_{2}^{\dagger}$.

Then the states $|k, N-k\rangle$ are separable: this agrees with the fact that they are created by the local operators $\left(a_{1}^{\dagger}\right)^{k}\left(a_{2}^{\dagger}\right)^{N-k}$. Indeed, for any polynomial operator $\mathcal{P}_{1} \in \mathcal{A}_{1}$ and $\mathcal{P}_{2} \in \mathcal{A}_{2}$, the expectation value of the product $\mathcal{P}_{1} \mathcal{P}_{2}$ is such that [compare with Eq. (42)]

$$
\begin{aligned}
\langle k, & \left.N-k\left|\mathcal{P}_{1} \mathcal{P}_{2}\right| k, N-k\right\rangle \\
\quad & =\frac{1}{k !(N-k) !}\left\langle 0\left|a_{1}^{k} \mathcal{P}_{1}\left(a_{1}^{\dagger}\right)^{k}\right| 0\right\rangle\left\langle 0\left|a_{2}^{N-k} \mathcal{P}_{2}\left(a_{2}^{\dagger}\right)^{N-k}\right| 0\right\rangle \\
& =\left\langle k\left|\mathcal{P}_{1}\right| k\right\rangle\left\langle N-k\left|\mathcal{P}_{2}\right| N-k\right\rangle,
\end{aligned}
$$

where $|k\rangle$ and $|N-k\rangle$ are single-mode Fock states. Consequently, mixed spearable states must be diagonal with respect to the Fock basis (45), i.e., density matrices of the form (Benatti, Floreanini, and Marzolino, 2010a)

$\rho=\sum_{k=0}^{N} p_{k}|k, N-k\rangle\langle k, N-k|, \quad p_{k} \geq 0, \quad \sum_{k=0}^{N} p_{k}=1$.

Unlike $\mathcal{P}_{1} \mathcal{P}_{2}$, most observables of physical interest are nonlocal with respect to the bipartition $\left(\mathcal{A}_{1}, \mathcal{A}_{2}\right)$, i.e., they are not of the form $\mathcal{O}=A_{1} A_{2}$, with $A_{1} \in \mathcal{A}_{1}$ and $A_{2} \in \mathcal{A}_{2}$. Prominent among them are those used in phase estimation protocols based on ultracold atoms trapped in double-well interferometers. Consider the generators of the rotations satisfying the $s u(2)$ algebraic relations $\left[J_{i}, J_{j}\right]=i \varepsilon_{i j k} J_{k}, i$, $j, k=x, y, z$. Among their possible representations in terms of two-mode creation and annihilation operators, let us focus upon the following:

$$
\begin{aligned}
& J_{x}=\frac{1}{2}\left(a_{1}^{\dagger} a_{2}+a_{1} a_{2}^{\dagger}\right), \\
& J_{y}=\frac{1}{2 i}\left(a_{1}^{\dagger} a_{2}-a_{1} a_{2}^{\dagger}\right), \\
& J_{z}=\frac{1}{2}\left(a_{1}^{\dagger} a_{1}-a_{2}^{\dagger} a_{2}\right) .
\end{aligned}
$$

Note that, although the operators in Eq. (48), as well as the exponentials $e^{i \theta J_{x}}$ and $e^{i \theta J_{y}}$, are nonlocal with respect to the bipartition $\left(\mathcal{A}_{1}, \mathcal{A}_{2}\right), \theta \in[0,2 \pi]$, the exponential of $J_{z}$ turns out to be local:

$$
e^{i \theta J_{z}}=e^{i \theta a_{1}^{\dagger} a_{1} / 2} e^{-i \theta a_{2}^{\dagger} a_{2} / 2},
$$

with $e^{i \theta a_{1}^{\dagger} a_{1} / 2} \in \mathcal{A}_{1}$ and $e^{-i \theta a_{2}^{\dagger} a_{2} / 2} \in \mathcal{A}_{2}$. By a linear transformation, one can always pass to new annihilation operators

$$
b_{1}=\frac{a_{1}+a_{2}}{\sqrt{2}}, \quad b_{2}=\frac{a_{1}-a_{2}}{\sqrt{2}},
$$

and corresponding creation operators $b_{1,2}^{\dagger}$, and rewrite the three operators in Eq. (48) as

$$
\begin{aligned}
& J_{x}=\frac{1}{2}\left(b_{1}^{\dagger} b_{1}-b_{2}^{\dagger} b_{2}\right), \\
& J_{y}=\frac{1}{2 i}\left(b_{1} b_{2}^{\dagger}-b_{1}^{\dagger} b_{2}\right), \\
& J_{z}=\frac{1}{2}\left(b_{1} b_{2}^{\dagger}+b_{1}^{\dagger} b_{2}\right) .
\end{aligned}
$$

To $b_{i}, b_{i}^{\dagger}, i=1,2$ one associates the bipartition $\left(\mathcal{B}_{1}, \mathcal{B}_{2}\right)$ of $\mathcal{A}$ consisting of the subalgebras generated by $b_{1}, b_{1}^{\dagger}$, respectively, $b_{2}, b_{2}^{\dagger}$ : with respect to it, the exponential of $J_{x}$ becomes

$$
e^{i \theta J_{x}}=e^{i \theta b_{1}^{\dagger} b_{1} / 2} e^{-i \theta b_{2}^{\dagger} b_{2} / 2},
$$

with $e^{i \theta b_{1}^{\dagger} b_{1} / 2} \in \mathcal{B}_{1}$ and $e^{-i \theta b_{2}^{\dagger} b_{2} / 2} \in \mathcal{B}_{2}$. This explicitly shows that an operator, local with respect to a given bipartition, can become nonlocal if a different algebraic bipartition is chosen.

These considerations are relevant for metrological applications (Caves, 1981; Yurke, 1986; Yurke, McCall, and Klauder, 1986; Holland and Burnett, 1993; Kitagawa and Ueda, 1993; Wineland et al., 1994; Sanders and Milburn, 1995; Bollinger et al., 1996; Bouyer and Kasevich, 1997; Dowling, 1998, 2008; Sørensen et al., 2001; Dunningham, Buenett, and Barnett, 2002; Holland, Kok, and Dowling, 2002; Wang and Sanders, 2003; Giovannetti, Lloyd, and Maccone, 2004, 2011; Korbicz, Cirac, and Lewenstein, 2005; Higgins et al., 2007; Uys and Meystre, 2007; Boixo et al., 2009; Dorner et al., 2009; Pezzè and Smerzi, 2009; Tóth et al., 2009; Kacprowicz et al., 2010). In fact, ultracold atoms trapped in a double-well optical potential realize a very accurate interferometric device: state preparation and beam splitting can be precisely achieved by tuning the interatomic interaction and by acting on the height of the potential barrier. The combination of standard MachZehnder-type interferometric operations, i.e., state preparation, beam splitting, phase shift, and subsequent beam recombination, can be effectively described as a suitable rotation of the initial state $\rho_{\text {in }}$ by a unitary transformation (Yurke, McCall, and Klauder, 1986; Sanders and Milburn, 1995):

$$
\rho_{\text {in }} \mapsto \rho_{\theta}=U_{\theta} \rho_{\text {in }} U_{\theta}^{\dagger}, \quad U_{\theta}=e^{i \theta J_{n}} .
$$

The phase change is induced precisely by the operators in Eq. (48) through the combination

$$
J_{n} \equiv n_{x} J_{x}+n_{y} J_{y}+n_{z} J_{z}, \quad n_{x}^{2}+n_{y}^{2}+n_{z}^{2}=1 .
$$

In practice, the state transformation $\rho_{\text {in }} \mapsto \rho_{\theta}$ inside the interferometer can be effectively modeled as a pseudospin rotation along the unit vector $\boldsymbol{n}=\left(n_{x}, n_{y}, n_{z}\right)$, whose choice depends on the specific realization of the interferometric apparatus and of the adopted measurement procedure.

As discussed in Sec. I, in the case of distinguishable particles, for any separable state $\rho_{\text {sep }}$ the quantum Fisher information is bounded by $I_{\theta}\left[\rho_{\text {in }}, J_{n}\right] \leq c N$ where $c$ is a constant independent of $N$ that can be taken as the maximum quantum Fisher information of a single system in all components of the mixed separable state [see Eq. (8)] (Fujiwara and Hashizumé, 2002; Giovannetti, Lloyd, and Maccone, 2006; Pezzè and Smerzi, 2009). Then entangled initial states of 
distinguishable particles are needed in order to obtain subshot-noise accuracies. A corresponding statement was proven by Benatti and Braun (2013) for a system of $N$ indistinguishable bosons: The quantum Fisher information with respect to a parameter $\theta$ imprinted onto a $(\mathcal{A}, \mathcal{B})$ separable state via a $(\mathcal{A}, \mathcal{B})$ local unitary operator of the form $U(\theta)=$ $\exp \left\{i \theta\left[A\left(a, a^{\dagger}\right)+B\left(b, b^{\dagger}\right)\right]\right\}$, where $A(B)$ are Hermitian functions of $a, a^{\dagger}\left(b, b^{\dagger}\right)$, strictly vanishes. Hence, in order to be able to estimate $\theta$ at all (and even more so to beat the standard quantum limit in terms of the total number of bosons), the separability of the input state or the locality of the unitary operator that imprints $\theta$ on the state needs to be broken. Fortunately, mode nonlocality is easily achieved, e.g., in quantum optics with a simple beam-splitter, without any particle interactions (see later).

In the case of the double-well system previously introduced, the operator algebras defined by $a_{1}^{\dagger}, a_{1}$ and $a_{2}^{\dagger}, a_{2}$, are the natural ones. With respect to them, a balanced Fock number state as in Eq. (45) of the form $\rho_{N / 2}=|N / 2, N / 2\rangle\langle N / 2, N / 2|$ is separable. By choosing the vector $\boldsymbol{n}$ in the plane orthogonal to the $z$ direction one computes $I_{\theta}\left[\rho_{N / 2}, J_{n}\right]=N^{2} / 2+N$, thus approaching the Heisenberg limit.

As a concrete example (Benatti and Braun, 2013), consider the action of a beam splitter described by the unitary $U_{B S}(\alpha)=\exp \left(\alpha a_{1}^{\dagger} a_{2}-\alpha^{*} a_{1} a_{2}^{\dagger}\right)$ involving two modes. If the complex transparency parameter $\alpha=i \theta / 2$, with $\theta$ real, then $U_{B S}(\alpha)=\exp \left(i \theta J_{x}\right)$. In the case of $N$ distinguishable qubits, a state as the balanced Fock number state $|N / 2, N / 2\rangle$ has half qubits in the state $|0\rangle$ and half in the state $|1\rangle$ such that $\sigma_{z}|i\rangle=(-)^{i}|i\rangle, i=0,1$. Then $J_{i}=\sum_{j=1}^{N} \sigma_{i}^{(j)} / 2$ with $i \in$ $\{x, y, z\}$ have zero mean values, while the purity of $\rho_{N / 2}$ yields $I_{\theta}\left[\rho_{N / 2}, J_{x}\right]=4\left\langle J_{x}^{2}\right\rangle=N$, since

$$
J_{x}^{2}=\frac{N}{4}+\frac{1}{4} \sum_{j \neq k=1}^{N} \sigma_{x}^{(j)} \sigma_{x}^{(k)}
$$

Instead, in the case of indistinguishable bosonic qubits, the mean values of $J_{i}$ for all $i \in\{x, y, z\}$ in Eq. (48) vanish, while using Eq. (48), $I_{\theta}\left[\rho_{N}, J_{x}\right]=4\left\langle J_{x}^{2}\right\rangle=N^{2} / 2+N$.

Unlike in the case of distinguishable particles, the quantum Fisher information can thus attain a value greater than $N$ even with initial states such as $\rho_{N / 2}$ that are separable with respect to the given bipartition. As mentioned before, the rotation operated by the beam splitter is not around the $z$ axis and is thus nonlocal with respect to the chosen bipartition. From the point of view of mode entanglement, it is thus not the entanglement of the states fed into the beam splitter that helps overcoming the shot-noise limit in the transparency parameter $\theta$ estimation accuracy; rather, the nonlocal character of the rotations operated by the apparatus on initially separable states allows $\sigma\left(\theta_{\text {est }}\right)$ to be smaller than $1 / \sqrt{N}$, with the possibility of eventually reaching the Heisenberg $1 / N$ limit (Benatti, Floreanini, and Marzolino, 2011; Benatti and Braun, 2013). Note that, if one does not take into account the identity of particles, the beam-splitter action in Eq. (56) is particle local according to the discussion at the beginning of Sec. I.A; indeed,

$$
e^{i \theta J_{x}}=\bigotimes_{j=1}^{N} e^{i \theta \sigma_{x}^{(j)} / 2}
$$

Thus, a prior massive entanglement of the input state

$$
|0\rangle \otimes \cdots|0\rangle \otimes|1\rangle \otimes \cdots|1\rangle
$$

with $k$ spins up, $\sigma_{z}|0\rangle=|0\rangle$, and $N-k$ spins down, $\sigma_{z}|1\rangle=-|1\rangle$, is needed.

Instead, if particle identity is considered, then the operator in Eq. (60) is not particle local since it cannot be written as a product of symmetrized single-particle operators [see Eq. (37) for the case $N=2$ ]. Furthermore, in the formalism of first quantization, a Fock number state as in Eq. (45) is represented in the symmetrized form (Benatti and Braun, 2013)

$$
\frac{1}{\mathcal{N}} \sum_{\pi}\left|0_{\pi(1)}\right\rangle \otimes \cdots\left|0_{\pi(k)}\right\rangle \otimes\left|1_{\pi(k+1)}\right\rangle \otimes \cdots\left|1_{\pi(N)}\right\rangle,
$$

where the sum is over all possible permutations $\pi$ of the $N$ indices and $\mathcal{N}=\sqrt{N ! k !(N-k) !}$. Despite its formally entangled structure, such a state is the symmetrization of a tensor product state with the first $k$ particles in the state $|0\rangle$ and the second $N-k$ particles in the state $|1\rangle$. Generalizing the argument briefly sketched in Sec. I.A in the case of two identical particles, individual properties can then be attributed to each of its constituents. Therefore, the state in Eq. (62) carries no particle entanglement, particle nonlocality being instead provided by the particle nonlocal operator in Eq. (60).

Of course, returning to the second quantization formalism, by changing bipartition from $\left(\mathcal{A}_{1}, \mathcal{A}_{2}\right)$ to $\left(\mathcal{B}_{1}, \mathcal{B}_{2}\right)$ via Eqs. (52), the action of the beam splitter, as outlined in Eq. (56), is local. In this bipartition, the nonlocality necessary for enhancing the sensitivity completely resides in the state. Therefore, the mode description leaves the freedom to locate the resources necessary to accuracy enhancing either in the entanglement of the state or in the nonlocality of the operations performed on it.

Benatti and Braun (2013) considered the paradigmatic case of phase estimation in a Mach-Zehnder interferometer with similar results: in the mode bipartition corresponding to the two modes after the first beam splitter, the phase shift operation in one arm is a local operator. Hence, at that stage the state must be mode entangled to allow estimating the phase shift with an accuracy better than the shot-noise limit. However, under certain conditions the first beam splitter can generate enough mode entanglement from a modeseparable state fed into the two input ports of the interferometer to beat the standard quantum limit. For more general settings, the question of what scaling of the quantum Fisher information can be achieved with the number of indistinguishable bosons is still open. Nonlocality is partially attributed to operations such as beam splitting instead of entirely to states, even in cases when fluffy-bunny entanglement is turned into useful entanglement as discussed by Killoran, Cramer, and Plenio (2014).

For massive bosons one might think that the tensor product of Fock states is a very natural state: As the boson number is conserved at the energies considered, one cannot make 
coherent superpositions of different numbers of atoms. However, in typical experiments, one needs to average over many runs, and the real difficulty exists in controlling the atom number with single-atom precision from run to run (Demkowicz-Dobrzański, Jarzyna, and Kołodiński, 2015). One has therefore effectively a mixed state with a distribution of different atom numbers. So far the best experimentally demonstrated approximations to Fock states with massive bosons are number-squeezed states with a few $\mathrm{dB}$ of squeezing (Esteve et al., 2008; Gross et al., 2010; Riedel et al., 2010). One can hope that novel measurement techniques such as the quantum gas microscope (Bakr et al., 2009) may enable precise knowledge of boson numbers in the future and thus the preparation of Fock states with a large number of atoms at least in a postselected fashion.

Recently, Oszmaniec et al. (2016) used the concentration of measure techniques to investigate the usefulness of randomly sampled probe states for unitary quantum metrology. They showed that random pure states drawn from the Hilbert space of distinguishable particles typically do not lead to superclassical scaling of precision. However, random states from the symmetric subspace, i.e., bosonic states, typically achieve the Heisenberg limit, even for very mixed isospectral states. Moreover, the quantum enhancement is typically robust against the loss of particles, in contrast to, e.g., GHZ states. It remains to be seen how entangled these states are in the sense of mode entanglement, but independently of the outcome of such an assessment, these results are in line with the finding that the naturally symmetrized pure states of bosons are a useful resource for quantum metrology. The fact that certain bosonic states can lead to the Heisenberg limit while mode entanglement does not play any significant role was also recently emphasized by Friis et al. (2015) and Šafránek and Fuentes (2016).

There are also metrological advantages achievable with bosons that are beyond the context of "standard quantum limit" versus Heisenberg limit scaling: Duivenvoorden, Terhal, and Weigand (2017) showed that by using a grid state (Gottesman, Kitaev, and Preskill, 2001) of a single bosonic mode, one can determine both amplitude and phase of a Fourier component of a small driving field that adds at most $\pi / 2$ photons, or, equivalently, both quadrature components of the displacement operator of the state. Slightly biased estimators were found whose sum of mean square deviations from the true values scales as $1 / \sqrt{n}$ with the average number of photons $n$ in the probe state. A "compass state" was proposed by Zurek (2001) that achieves similar sensitivity for small displacements up to the order of $1 / \sqrt{n}$. These results should be contrasted with the lower bound for any single-mode Gaussian state as a probe state that is of the order of 1 , independent of $n$, and regardless of the amount of squeezing. With two-mode Gaussian states one can beat this constant lower bound, but then the two modes must be necessarily entangled (Genoni et al., 2013). It is possible that the result by Duivenvoorden, Terhal, and Weigand (2017) may still be improved upon with other states, as in Duivenvoorden, Terhal, and Weigand (2017) also a lower bound for all single-mode probe states was found that scales as $1 /(2 n+1)$. This is the same lower bound as for arbitrary (in particular, entangled) two-mode Gaussian states (Genoni et al., 2013).

\section{Mode entanglement and metrology: Fermions}

As in the case of bosonic systems, we shall consider generic fermionic many-body systems made of $N$ elementary constituents that can occupy $\mathfrak{M}$ different states or modes $N<\mathfrak{M}$. The creation $a_{i}^{\dagger}$ and annihilation $a_{i}$ operators for mode $i$ obey now the anticommutation relations $\left\{a_{i}, a_{j}^{\dagger}\right\}=\delta_{i j}$ and generate the fermion algebra $\mathcal{A}$, i.e., the norm closure of all polynomials in these operators. As already specified, a bipartition $\left(\mathcal{A}_{1}, \mathcal{A}_{2}\right)$ of this algebra is the splitting of the collection of creation and annihilation operators into two disjoint sets. The Hilbert space $\mathcal{H}$ of the system is again generated out of the vacuum state $|0\rangle$ by the action of the creation operators; it is spanned by the many-body Fock states,

$$
\left|n_{1}, n_{2}, \ldots, n_{\mathfrak{M}}\right\rangle=\left(a_{1}^{\dagger}\right)^{n_{1}}\left(a_{2}^{\dagger}\right)^{n_{2}} \cdots\left(a_{\mathfrak{M}}^{\dagger}\right)^{n_{\mathfrak{M}}}|0\rangle,
$$

where the integers $n_{1}, n_{2}, \ldots, n_{\mathfrak{M}}$ are the occupation numbers of the different modes, with $\sum_{i} n_{i}=N$; they can now take only the two values 0 or 1 .

As already clear from the definition of fermionic algebraic bipartitions, because of the anticommutation relations, in dealing with fermions, one must distinguish between even and odd operators. While the even component $\mathcal{A}^{e}$ of $\mathcal{A}$ consists of elements $A^{e} \in \mathcal{A}$ such that $\vartheta\left(A^{e}\right)=A^{e}$, the odd component $\mathcal{A}^{o}$ of $\mathcal{A}$ consists of those elements $A^{o} \in \mathcal{A}$ such that $\vartheta\left(A^{o}\right)=-A^{o}$. Even elements of $\mathcal{A}$ commute with all other elements, while odd elements commute only with even ones.

The anticommuting character of the fermion algebra $\mathcal{A}$ puts stringent constraints on the form of the fermion states that can be represented as product of other states, such as the ones appearing in the decomposition (40) that defines separable states. Specifically, as a consequence of the result in Araki and Moriya (2003), any product $\omega_{k}^{(1)}\left(A_{1}\right) \omega_{k}^{(2)}\left(A_{2}\right)$ vanishes whenever $A_{1}$ and $A_{2}$ both belong to the odd components of their respective subalgebras. Then given a mode bipartition $\left(\mathcal{A}_{1}, \mathcal{A}_{2}\right)$ of the fermionic algebra $\mathcal{A}$, i.e., a decomposition of $\mathcal{A}$ in the subalgebra $\mathcal{A}_{1}$ generated by the first $m$ modes and the subalgebra $\mathcal{A}_{2}$, generated by the remaining $\mathfrak{M}-m$ ones, it follows that the decomposition (40) is meaningful only for local operators $A_{1} A_{2}$ for which $\left[A_{1}, A_{2}\right]=0$, so that the definition of separability it encodes is completely equivalent to the one adopted for bosonic systems.

As a further consequence of the result in Araki and Moriya (2003), one derives that if a state $\omega$ is nonvanishing on a local operator $A_{1}^{o} A_{2}^{o}$, with the two components $A_{1}^{o} \in \mathcal{A}_{1}^{o}, A_{2}^{o} \in \mathcal{A}_{2}^{o}$ both belonging to the odd part of the two subalgebras, then $\omega$ is entangled with respect to the bipartition $\left(\mathcal{A}_{1}, \mathcal{A}_{2}\right)$. Indeed, if $\omega\left(A_{1}^{o} A_{2}^{o}\right) \neq 0$, then $\omega$ cannot be written as in Eq. (40) and therefore cannot be separable.

Using these results, as for the bosonic case, one shows that (Benatti, Floreanini, and Marzolino, 2014), given a bipartition of the fermionic algebra $\mathcal{A}$ determined by the integer $m$, a pure state $|\psi\rangle$ results separable if and only if it can be written in the form (44). Examples of pure separable states of $N$ fermions are the Fock states in Eq. (63); indeed, they can be recast in the form 


$$
\begin{aligned}
& \left|k_{1}, \ldots, k_{m} ; p_{m+1}, \ldots, p_{\mathfrak{M}}\right\rangle \\
& \quad=\left[\left(\hat{a}_{1}^{\dagger}\right)^{k_{1}} \cdots\left(\hat{a}_{m}^{\dagger}\right)^{k_{m}}\right]\left[\left(\hat{a}_{m+1}^{\dagger}\right)^{p_{m+1}} \cdots\left(\hat{a}_{\mathfrak{M}}^{\dagger}\right)^{p_{\mathfrak{M}}}\right]|0\rangle,
\end{aligned}
$$

where the $\mathcal{P}$ and $\mathcal{Q}$ appearing in Eq. (44) are now monomials in the creation operators of the two partitions.

Concerning the metrological use of fermionic systems, the situation may appear more problematic than with bosons, for each mode can accommodate at most one fermion; therefore, the scaling with $N$ of the sensitivity in the estimation of physical parameters may worsen. Indeed, while a two-mode bosonic apparatus, as a double-well interferometer, filled with $N$ particles is sufficient to reach sub-shot-noise sensitivities, with fermions, a multimode interferometer is needed in order to reach comparable sensitivities [for the use of multimode interferometers see, for instance, D'Ariano and Paris (1997), D'Ariano, Macchiavello, and Sacchi (1998), Söderholm et al. (2003), Vourdas and Dunningham (2005), and Cooper, Hallwood, and Dunningham (2009), and Cooper et al. (2012) in the fermionic case]. As an example, consider a system of $N$ fermions in $\mathfrak{M}$ modes, with $\mathfrak{M}$ even, and let us fix the balanced bipartition $(\mathfrak{M} / 2, \mathfrak{M} / 2)$, in which each of the two parts contains $m=\mathfrak{M} / 2$ modes, taking for simplicity $N \leq m$. As generator of the unitary transformation $\rho \rightarrow \rho_{\theta}$ inside the measuring apparatus let us take the following operator:

$$
\mathcal{J}=\frac{1}{2} \sum_{k=1}^{m} \omega_{k}\left(a_{k}^{\dagger} a_{m+k}+a_{m+k}^{\dagger} a_{k}\right)
$$

where $\omega_{k}$ is a given spectral function $\omega_{k} \simeq k^{p}$, with $p$ positive. The apparatus implementing this state transformation is nonlocal with respect to the chosen bipartition: $e^{i \theta \mathcal{J}}$ cannot be written as the product $A_{1} A_{2}$ of two components made of operators $A_{1}$ and $A_{2}$ belonging only to the first and second partitions, respectively. It represents a generalized, multimode beam splitter, and the whole measuring device behaves as a multimode interferometer.

Let us feed the interferometer with a pure initial state $\rho=|\psi\rangle\langle\psi|$,

$$
|\psi\rangle=|\underbrace{1, \ldots, 1}_{N}, \underbrace{0, \ldots, 0}_{m-N} ; \underbrace{0, \ldots, 0}_{m}\rangle=a_{1}^{\dagger} a_{2}^{\dagger} \cdots a_{N}^{\dagger}|0\rangle,
$$

where the fermions occupy the first $N$ modes of the first partition; $|\psi\rangle$ is a Fock state and therefore it is separable, as already discussed. The quantum Fisher information can easily be computed (Benatti, Floreanini, and Marzolino, 2014)

$$
I_{\theta}[\rho, \mathcal{J}]=\sum_{k=1}^{N} \omega_{k}^{2}
$$

Unless $\omega_{k}$ is $k$ independent, $I_{\theta}[\rho, \mathcal{J}]$ is larger than $N$ and therefore the interferometric apparatus can beat the shot-noise limit in the $\theta$ estimation, even starting with a separable state. Actually, for $\omega_{k} \simeq k^{p}$, one gets $I_{\theta}[\rho, \mathcal{J}] \simeq N^{2 p+1}$, reaching sub-Heisenberg sensitivities with a linear device. Note that this result and the ability to go beyond the Heisenberg limit is not a "geometrical" phenomenon attributable to a phase accumulation even on empty modes (D'Ariano and Paris, 1997); rather, it is a genuine quantum effect that scales as a function of the number of fermions, the resource available in the measure.

Again, as in the bosonic case, it is not the entanglement of the initial state that helps to overcome the shot-noise limit in the phase estimation; rather, it is the nonlocal character of the rotations operated by the apparatus on an initially separable state that allows one to beat the shot-noise limit.

\section{MORE GENERAL HAMILTONIANS}

\section{A. Nonlinear Hamiltonians}

Most discussions of quantum-enhanced measurements consider, implicitly or explicitly, evolution under a Hamiltonian that is linear in a collective variable of the system. For illustration, consider Ramsey spectroscopy on a collection of $N$ atoms with ground and excited states $|g\rangle$ and $|e\rangle$, respectively. If these have energies $\pm \hbar \omega / 2$, then the Hamiltonian giving rise to the Ramsey oscillation is

$$
H=\hbar \omega S_{z} \equiv \hbar \omega \sum_{i=1}^{N} s_{z}^{(i)}
$$

where $s_{z} \equiv(1 / 2)(|e\rangle\langle e|-| g\rangle\langle g|)$ is a pseudospin-1/2 operator describing the transition and the superscript ${ }^{(i)}$ indicates the $i$ th atom. This Hamiltonian is manifestly linear in $S_{z}$ and can be trivially decomposed into micro-Hamiltonians $H=$ $\sum_{i} h^{(i)} \equiv \sum_{i} \hbar \omega s_{z}^{(i)}$ that describe the uncoupled precession of each atom in the ensemble. For a single Ramsey sequence of duration $T$, so that the unknown parameter is $\theta=\omega T$, the standard quantum limit and Heisenberg-limit sensitivities are as described in Sec. I, $\operatorname{Var}\left(\theta_{\mathrm{est}}\right)_{\mathrm{SQL}}=N^{-1}$ and $\operatorname{Var}\left(\theta_{\text {est }}\right)_{\mathrm{HL}}=N^{-2}$. The assumption of uncoupled particles is often physically reasonable, for example, when describing photons in a linear interferometer or low-density atomic gases for which collisional interactions can be neglected. In other systems, including Bose-Einstein condensates (Gross et al., 2010; Riedel et al., 2010), laser interferometers at high power (The LIGO Scientific Collaboration, 2011; Aasi et al., 2013), high-density atomic magnetometers (Kominis et al., 2003; Dang, Maloof, and Romalis, 2010; Shah, Vasilakis, and Romalis, 2010; Vasilakis, Shah, and Romalis, 2011) and high-density atomic clocks (Deutsch et al., 2010), the assumption of uncoupled particles is unwarranted. This motivates the study of nonlinear Hamiltonians.

The unusual features of nonlinear Hamiltonians are well illustrated in the following example (Boixo, Datta, Flammia et al., 2008). First, define the collective operator $S_{0} \equiv \sum_{i} s_{0}^{(i)} \equiv$ $\sum_{i} \mathbb{1}^{(i)}$, where $\mathbb{1}$ indicates the identity operator. $S_{0}$ is clearly the total number of particles. Now consider the nonlinear Hamiltonian

$$
H=\hbar \Omega S_{0} S_{z}=\hbar \Omega \sum_{i=1}^{N} \sum_{j=1}^{N} s_{0}^{(i)} s_{z}^{(j)}
$$


This is linear in the unknown $\Omega$, but of second order in the collective variables $S$. At the microscopic level, the Hamiltonian describes a pairwise interaction, with energy $\hbar \Omega s_{0}^{(i)} s_{z}^{(j)}$, between each pair of particles $(i, j)$.

For a system with a fixed number $N$ of particles, the consequence for the dynamics of the system is very simple: the operator $S_{0}$ can be replaced by its eigenvalue $N$ leading to an effective Hamiltonian

$$
H_{N}=\hbar N \Omega S_{z}
$$

Estimation of the product $N \Omega T=N \Theta$ now gives the same uncertainties that we saw earlier in the estimation of $\theta$. That is, $\operatorname{Var}\left(N \Theta_{\text {est }}\right)_{\mathrm{SQL}}=N^{-1}$ so that $\operatorname{Var}\left(\Theta_{\text {est }}\right)_{\mathrm{SQL}}=N^{-3}$. Similarly, $\operatorname{Var}\left(\Theta_{\text {est }}\right)_{\mathrm{HL}}=N^{-4}$. More generally, a nonlinear Hamiltonian containing $k$-order products of collective variables will contain $N^{k}$ microscopic interaction terms that contribute to the Hamiltonian and thus to the rate of change of an observable such as $S_{z}$ under time evolution. In contrast, the variance of such a macroscopic observable, e.g., $\operatorname{Var}\left(S_{z}\right)$, scales as $N^{1}$ (standard quantum limit) or $N^{0}$ (Heisenberg limit). This allows signal-to-noise ratios scaling as $N^{2 k-1}$ (standard quantum limit) and as $N^{2 k}$ (Heisenberg limit) (Boixo et al., 2007). These noise terms depend only on the size of the system and nature of the initial state and not on the Hamiltonian (Boixo et al., 2007).

That nonlinearities lead to improved scaling of the sensitivity appears to have been independently discovered by Luis (2004, 2007) and Beltrán and Luis (2005) and by Boixo et al. (2007), Boixo, Datta, Davis et al. (2008), Boixo, Datta, Flammia et al. (2008), and Datta and Shaji (2012). A related proposal using interactions to give a scaling $\sigma\left(\theta_{\text {est }}\right) \propto 2^{-N}$ is described by Roy and Braunstein (2008).

Prior to the appearance of these results, the term Heisenberg limit, which was introduced in the literature by Holland and Burnett (1993) in the context of interferometric phase estimation with the definition $\sigma\left(\phi_{\text {est }}\right)=1 / N$, had been used, often indiscriminately, to describe (1) the sensitivity $\sigma\left(\phi_{\text {est }}\right)=1 / N$, (2) the scaling $\sigma\left(\phi_{\text {est }}\right) \propto 1 / N$, (3) the best possible sensitivity with $N$ particles, and (4) the best possible scaling with $N$ particles (Giovannetti, Lloyd, and Maccone, 2004, 2006, 2011). These multiple definitions are not all compatible in a scenario with a nonlinear Hamiltonian. Taking as a definition "error ... bounded by the inverse of the physical resources," and implicitly considering scaling, Zwierz, PérezDelgado, and Kok (2010) [see also Zwierz, Pérez-Delgado, and Kok (2011)] showed that an appropriate definition for a "physical resource" is the query complexity of the system viewed as a quantum network.

For the simplest optical nonlinearity, $\theta \propto N^{2}$ and quadrature detection, it has been shown that quadrature squeezed states are near optimal (Maldonado-Mundo and Luis, 2009). Considering the same nonlinearity and limiting to classical inputs, i.e., coherent states and mixtures thereof, it was argued by Rivas and Luis (2010) that nonlinear strategies can outperform linear ones by concentrating the available particles in a small number of high-intensity probes. Tilma et al. (2010) analyzed a variety of entangled coherent states for nonlinear interferometry of varying orders and found that in most cases entanglement degraded the sensitivity for high-order nonlinearities. Berrada (2013) considered the use of two-mode squeezed states as inputs to a nonlinear interferometer, including the effects of loss, and showed a robust advantage for such states.

As already mentioned in Sec. I.C, these results concern local measures implying some prior knowledge of the parameter being estimated. The situation for global measures without prior information was considered by Hall and Wiseman (2012).

\section{B. Proposed experimental realizations}

A number of physical systems have been proposed for nonlinear quantum-enhanced measurements: Propagation through nonlinear optical materials (Luis, 2004, 2007; Beltrán and Luis, 2005), collisional interactions in BoseEinstein condensates (Boixo et al., 2009), Duffing nonlinearity in nanomechanical resonators (Woolley, Milburn, and Caves, 2008), and nonlinear Faraday rotation probing of an atomic ensemble (Napolitano and Mitchell, 2010).

\section{Nonlinear optics}

The first proposals concerned nonlinear optics (Luis, 2004, 2007; Beltrán and Luis, 2005), in which a nonlinear optical susceptibility is directly responsible for a phase shift $\theta \propto N^{k}$, where $k$ is the order of the nonlinear contribution to the refractive index. In the simplest example, Beltrán and Luis (2005) showed that an input coherent state $|\alpha\rangle$, experiencing a Kerr-type nonlinearity described by the unitary $\exp \left[i \Theta\left(a^{\dagger} a\right)^{2}\right]$, and detected in quadrature $X=(1 / \sqrt{2})\left(a+a^{\dagger}\right)$, gives an outcome distribution

$$
P(X=x \mid \Theta)=\left|\left\langle x\left|e^{i \Theta\left(a^{\dagger} a\right)^{2}}\right| \alpha\right\rangle\right|^{2}
$$

If we consider the case of small $\Theta$, imaginary $\alpha=i \sqrt{\langle N\rangle}$, and the estimator $\Theta_{\text {est }}=\bar{X} /|\partial \bar{X} / \partial \theta|$, where $\bar{X} \equiv \sum_{i=1}^{M} X_{i}$ is the mean of the observed quadratures, we find the standard deviation

$$
\begin{aligned}
\sigma\left(\Theta_{\text {est }}\right) & =\frac{\sigma(X)}{\sqrt{M}|d\langle X\rangle / d \Theta|}=\frac{\sigma(X)}{\sqrt{M}\left|\left\langle\left[X,\left(a^{\dagger} a\right)^{2}\right]\right\rangle\right|} \\
& =\frac{1}{4 M^{1 / 2} N^{-3 / 2}} .
\end{aligned}
$$

Here we used $\sigma(X)=1 / \sqrt{2}$ for the quantum mechanical uncertainty of $X$ in the initial state, and which up to corrections of order $\Theta^{2}$ holds also for the evolved state when $\Theta$ is small. For large $N$, this strategy saturates the quantum Cramér-Rao bound; the quantum Fisher information is straightforwardly calculated to be $I_{\Theta}=4 N+24 N^{2}+16 N^{3}$.

\section{Ultracold atoms}

Coherent interaction-based processes are well developed in Bose-Einstein condensates and have been used extensively for squeezing generation. For example, a confined two-species 
Bose-Einstein condensate experiences collisional energy shifts described by an effective Hamiltonian

$$
\begin{aligned}
H_{\mathrm{eff}} & \propto a_{11} n_{1}\left(n_{1}-1\right)+2 a_{12} n_{1} n_{2}+a_{22} n_{2}\left(n_{2}-1\right) \\
= & \left(a_{11}-2 a_{12}+a_{22}\right) S_{z}^{2}+2\left(a_{11}-a_{22}\right) S_{z} S_{0} \\
& + \text { terms in } S_{z}, S_{0}
\end{aligned}
$$

where $S_{z} \equiv(1 / 2)\left(n_{1}-n_{2}\right)$ and $S_{0} \equiv(1 / 2)\left(n_{1}+n_{2}\right)$ are pseudospin operators, $n_{1}$ and $n_{2}$ are the number of atoms of species 1 and 2, respectively, and $a_{i j}$ are the collisional scattering lengths. In ${ }^{87} \mathrm{Rb}$, and with $|1\rangle \equiv|F=1, m=-1\rangle$, $|2\rangle \equiv|F=2, m=1\rangle$, the scattering lengths (near zero magnetic field) have the ratio $a_{11}: a_{12}: a_{22}=1.03: 1: 0.97$. A proven method to generate spin squeezing in this system is to increase $a_{12}$ using a Feshbach resonance to give the singleaxis twisting Hamiltonian $H_{\text {eff }} \propto S_{z}^{2}$ (Muessel et al., 2014), plus terms proportional to $S_{z}$ and $S_{0}$, which induce a global rotation and a global phase shift, respectively.

Boixo, Datta, Davis et al. (2008) observed that the zerofield scattering lengths naturally give $a_{11}-2 a_{12}+a_{22} \approx 0$, making small the coefficient of $S_{z}^{2}$ and leaving the $S_{0} S_{z}$ term as the dominant nonlinear contribution. Detailed analyses of the Bose-Einstein condensate physics beyond the simplified single-mode treatment here are given by Boixo, Datta, Flammia et al. (2008), Boixo et al. (2009), and Tacla and Caves (2013). The strategy gives $N^{-3 / 2}$ scaling for estimation of the relative scattering length $a_{11}-a_{22}$. Mahmud, Tiesinga, and Johnson (2014) described a strategy of dynamical decoupling to suppress the second-order terms in the Hamiltonian and thus make dominant three-body interactions, giving a sensitivity scaling of $N^{-5 / 2}$ for measurements of three-body collision strengths.

\section{Nanomechanical oscillators}

Woolley, Milburn, and Caves (2008) proposed a nonlinear interferometer using two modes of a nanomechanical oscillator, with amplitudes $x_{a}$ and $x_{b}$, experiencing the nonlinear Hamiltonian

$H_{\mathrm{eff}}=H_{\mathrm{SHO}}^{(a)}+H_{\mathrm{SHO}}^{(b)}+\frac{1}{4} \chi_{a} m \omega^{2} x_{a}^{4}+\frac{1}{4} \chi_{b} m \omega^{2} x_{b}^{4}+C(t)$,

where $H_{\mathrm{SHO}}$ indicates the simple harmonic oscillator Hamiltonian (SHO), $\chi$ is the Duffing nonlinearity coefficient, $\omega$ is the low-amplitude resonance frequency, and $C$ is an externally controlled coupling between modes $a$ and $b$ that produces a beam-splitter interaction. With an interferometric sequence resembling a Mach-Zehnder interferometer, the Duffing nonlinearity can be estimated with uncertainty scaling as $N^{-3 / 2}$, where $N$ is the number of excitations.

\section{Nonlinear Faraday rotation}

Whereas Luis and co-workers considered phenomenological models of optical nonlinearities, Napolitano and Mitchell (2010) described an $a b$ initio calculation of the optical nonlinearity produced on a particular atomic transition, using degenerate perturbation theory and a collective quantum variable description. This gives an effective Hamiltonian for the interaction of polarized light, described by the Stokes operators $\mathbf{S}$, with the collective orientation and alignment spin variables $\mathbf{J}$ of an atomic ensemble:

$$
\begin{gathered}
H_{\mathrm{eff}}=H_{\mathrm{eff}}^{(2)}+H_{\mathrm{eff}}^{(4)}+O\left(\mathbf{S}^{3}\right), \\
H_{\mathrm{eff}}^{(2)}=\alpha^{(1)} S_{z} J_{z}+\alpha^{(2)}\left(S_{x} J_{x}+S_{y} J_{y}\right), \\
H_{\mathrm{eff}}^{(4)}=\beta_{J}^{(0)} S_{z}{ }^{2} J_{0}+\beta_{N}^{(0)} S_{z}{ }^{2} N_{A}+\beta^{(1)} S_{0} S_{z} J_{z} \\
+\beta^{(2)} S_{0}\left(S_{x} J_{x}+S_{y} J_{y}\right),
\end{gathered}
$$

where the $\alpha$ and $\beta$ coefficients are linear and nonlinear polarizabilities that depend on the detuning of the probe photons from the atomic resonance. By proper choice of detuning and initial atomic polarization $\mathbf{J}$, the term $\beta^{(1)} S_{0} S_{z} J_{z}$ can be made dominant, making the Hamiltonian formally equivalent to that of Eq. (69). Note that $\beta^{(1)} J_{z}$, proportional to the atomic polarization $J_{z}$, plays the role of the unknown interaction energy $\hbar \Omega$. The photons are thus made to interact, mediated by and proportional to the atomic polarization $J_{z}$. For a different detuning, the term $\alpha^{(1)} S_{z} J_{z}$ becomes dominant, allowing a linear measurement of the same quantity $J_{z}$ with the same atomic system.

The experimental realization using a cold, optically trapped ${ }^{87} \mathrm{Rb}$ atomic ensemble is described by Napolitano et al. (2011). The experiment observed the predicted scaling of $\operatorname{Var}\left(J_{z}\right) \propto N^{-3}$ over a range of photon numbers from $N=$ $5 \times 10^{5}$ to $5 \times 10^{7}$. For larger photon numbers the scaling worsened, i.e., $\operatorname{Var}\left(J_{z}(N)\right)$ had a logarithmic derivative $>-3$. Because of this limited range of the $N^{-3}$ scaling, and the difference in prefactors $\beta^{(1)}$ vs $\alpha^{(1)}$, the nonlinear estimation never surpassed the sensitivity $\operatorname{Var}\left(J_{z}\right)$ of the linear measurement for the same number of photons. Nonetheless, due to a shorter measurement time $\tau$, the nonlinear measurement did surpass the linear measurement in spectral noise density $\operatorname{Var}\left(J_{z}\right) \tau$, a common figure of merit for time- or frequencyresolved measurements.

\section{Observations and commentary}

Several differences between linear and nonlinear strategies, perhaps surprising, deserve comment. First, it should be obvious that there is no conflict with the Heisenberg uncertainty principle. $\theta$ and $\Theta$ are parameters, not observables, and as such are not subject to operator-based uncertainty relations, neither the Heisenberg uncertainty principle nor generalizations such as the Robertson uncertainty relation (Robertson, 1929). Moreover, the advantageous scaling in $\delta \Theta$ is the result of a rapidly growing signal, rather than a rapidly decreasing statistical noise. A nonlinear Hamiltonian immediately leads to a strong change in the scaling of the signal: even the simplest $k=2$ nonlinearity gives signal growing as $\omega \propto N^{2}$ and thus standard quantum limit uncertainty $(\delta \Theta)_{\mathrm{SQL}} \propto$ $N^{-3 / 2}$, which scales faster with $N$ than does $(\delta \theta)_{\mathrm{HL}} \propto N^{-1}$.

Second, the estimated phases $\theta$ and $\Theta$ necessarily reflect different physical quantities. $\hbar \omega$ describes a single-particle energy such as that due to an external field, whereas $\hbar \Omega$ 
describes a pairwise interaction energy. As such, the uncertainties $\delta \theta$ and $\delta \Theta$ are not directly comparable. Any comparison of the efficacy of the measurements must introduce another element, a connection between a third physical quantity $\theta$ and $\Theta$. This we have seen in Sec. IV.B.4, where the unknown $J_{z}$ appears in both the linear and nonlinear Hamiltonians. In what follows, a comparison of optimized linear and nonlinear metrology is described.

\section{Nonlinear measurement under number-optimized conditions}

A more extensive study of nonlinear spin measurements using the same system as Napolitano et al. (2011) was reported by Sewell et al. (2014). This work compared two estimation strategies, one linear and one nonlinear, for measuring the collective variable $J_{y}$, which describes a component of the spin alignment tensor used in a style of optical magnetometry known as alignment-to-orientation conversion (Budker et al., 2000; Pustelny et al., 2008; Sewell et al., 2012). The linear estimation used the term $\alpha^{(2)} S_{y} J_{y}$, which appears in Eq. (78) and produces a rotation from linearly polarized light toward elliptically polarized light. The nonlinear estimation in contrast used Eq. (78) in second order: in the first step, due to the $\alpha^{(2)} S_{x} J_{x}$ term and the input $S_{x}$ optical polarization, an initial $J_{y}$ atomic polarization is rotated toward $J_{z}$ by an angle $\phi \propto\left\langle S_{x}\right\rangle$, and thus $\propto N$, where $N$ is the number of photons. In the second step, the term $\alpha^{(1)} S_{z} J_{z}$ produces a Faraday rotation, i.e., from $S_{x}$ toward $S_{y}$, by an angle proportional to the $J_{z}$ polarization produced in the first step. The resulting $S_{y}$ polarization is $S_{y} \propto J_{y} N^{2}$, while the statistical noise is $\sigma\left(S_{y}\right) \propto N^{1 / 2}$, giving sensitivity scaling $\sigma\left(J_{y}\right) \propto N^{-3 / 2}$. Importantly, the two estimation strategies use the same $S_{x}$-polarized input and thus have identical statistical noise and cause identical damage in the form of spontaneous scattering, which adds noise to the atomic polarization.

The experimentally observed nonlinear sensitivity was compared against the calculated ideal sensitivity of the linear measurement. Owing to its faster scaling, and more favorable prefactors than in Napolitano et al. (2011), the nonlinear measurement's sensitivity surpassed that of the ideal linear measurement at about $2 \times 10^{7}$ photons. A comparison was also made when each measurement was independently optimized for number $N$ and detuning, which affects both the prefactors $\alpha$ and the scattering. The ability to produce measurement-induced spin squeezing was taken as the figure of merit, and the fully optimized nonlinear measurement gave more squeezing than the fully optimized linear measurement. This shows that, for some quantities of practical interest, a nonlinear measurement can outperform the best possible linear measurement. Similar conclusions have been drawn for the case of number-optimized saturable spectroscopy (Mitchell, 2017).

\section{E. Signal amplification with nonlinear Hamiltonians}

The single-axis twisting Hamiltonian $H_{\text {twist }}=\chi S_{z}^{2}$, in addition to producing spin squeezed states, has been proposed as a nonlinear amplifier to facilitate state readout in atom interferometry (Davis, Bentsen, and Schleier-Smith, 2016).
Starting from an $x$-polarized coherent spin state $|\mathbf{x}\rangle$, and defining the unitary $U \equiv \exp \left[-i H_{\text {twist }} \tau / \hbar\right]$, the Wigner distribution of the squeezed state $U|\mathbf{x}\rangle$ is thin in the $z$ direction, and is thus sensitive to rotations $\mathcal{R}_{y}(\phi)$ about the $y$ axis, so that states of the form $\mathcal{R}_{y}(\phi) U|\mathbf{x}\rangle$ have large quantum Fisher information with respect to $\phi$. Exploitation of this in-principle sensitivity is challenging, however, because it requires lownoise readout, detecting $S_{z}$ at the single-atom level if the Heisenberg limit is to be approached. In contrast, a sequence that applies $H_{\text {twist }}$, waits for rotation about the $y$ axis, and then applies $-H_{\text {twist }}$ for an equal time generates the state $U^{\dagger} \mathcal{R}_{y}(\phi) U|\mathbf{x}\rangle$. Because $U^{\dagger}$ is unitary, the quantum Fisher information is unchanged, but the perturbation implied by $\mathcal{R}_{y}(\phi)$ now manifests itself at the scale of the original coherent spin state, which is to say it is amplified from the Heisenberglimit scale up to the standard quantum limit scale, greatly facilitating detection. Implementations include a cold-atom cavity QED system (Hosten et al., 2016) and Bose-Einstein condensates (Linnemann et al., 2016). While this strategy clearly uses entanglement, it is nonetheless striking that undoing the entanglement-generation step provides an important benefit.

\section{F. Other modifications of the Hamiltonian}

The assumption of a Hamiltonian $H=\sum_{k=1}^{N} h_{k}$ considered for the derivation of Eq. (1), where $\Lambda$ and $\lambda$ are the largest and smallest eigenvalues of $h_{k}$, respectively, not only implies distinguishable subsystems, it is also restrictive in two other important regards: (a) The existence of such bounds on the spectrum of $h_{k}$ may not be warranted, and (b) interactions between the subsystems are excluded. In this section we explore the consequences of lifting these restrictions.

\section{Lifting spectral limitations}

A large portion of the work on quantum-enhanced measurements stems from quantum optics, where the basic dynamical objects are modes of the electromagnetic (e.m.) field, corresponding to simple harmonic oscillators $h_{k}=$ $\hbar \omega_{k}\left(n_{k}+1 / 2\right)$. A phase shift in mode $k$ can be implemented by $U=\exp \left(\operatorname{in}_{k} \theta\right)$. For the relevant Hamiltonian $h_{k}=n_{k}$ acting as a generator of the phase shift, $\Lambda=\infty$ and $\lambda=0$. Hence, Eq. (1) implies a minimal uncertainty $\operatorname{Var}\left(\theta_{\text {est }}\right)=0$. Of course, one may argue that in reality one can never use states of infinite energy, such that there is effectively a maximum energy. However, it need not be that the maximum energy sustainable by the system must be distributed over $N$ modes. Indeed, what is typically counted in quantum optics in terms of resources is not the number of modes $N$, but the total number of photons $n$, directly linked to the total energy. It turns out that the total number of modes (or subsystems, in general) is completely irrelevant for achieving optimal sensitivity, even if the parameter is coded in several modes or subsystems, e.g., with a general unitary transformation of the form $U=\exp \left(i \theta \sum_{k=1}^{N} h_{k}\right)$, if one can stock the same amount of energy in a single system as in the total system. Note that this is often the case in quantum optics, where different modes can be spatially confined or parametrically influenced by the same optical elements such as mirrors, beam splitters, and 
phase shifters whose material properties ultimately determine the maximum amount of energy that can be used.

To see the liberating effect of unbound spectra, recall that for any initial pure state $|\psi\rangle$ propagated by a Hamiltonian of the form $H=\theta G$ with a Hermitian generator $G$ for a time $T$ the quantum Fisher information is given by (Braunstein and Caves, 1994; Braunstein, Caves, and Milburn, 1996)

$$
I_{\theta}=4 \operatorname{Var}(G) T^{2} \equiv 4\left(\left\langle G^{2}\right\rangle-\langle G\rangle^{2}\right) T^{2} .
$$

Let $G=\sum_{i} e_{i}|i\rangle \mid\langle i|$ be the spectral decomposition of $G$, and $|\psi\rangle=\sum_{i=1}^{L} c_{i}|i\rangle$, where we assume that $|1\rangle(|L\rangle)$ are the states of lowest (largest) energy available. Then $\operatorname{Var}(G)=$ $\sum_{i=1}^{L} p_{i} e_{i}^{2}-\left(\sum_{i=1}^{L} p_{i} e_{i}\right)^{2}$ with $p_{i}=\left|c_{i}\right|^{2}$ and $\sum_{i=1}^{L} p_{i}=1$. The Popoviciu (1935) inequality states $\operatorname{Var}(G) \leq$ $\left(e_{L}-e_{1}\right)^{2} / 4$. It is saturated for $p_{1}=p_{L}=1 / 2, p_{i}=0$ else. The state $|\psi\rangle=\left(|1\rangle+e^{i \varphi}|L\rangle\right) / \sqrt{2}$ with an arbitrary phase $\varphi$ saturates the inequality and thus maximizes $I_{\theta}$. If $e_{L}$ or $e_{1}$ is degenerate, only the total probability for the degenerate energy levels is fixed to $1 / 2$, and arbitrary linear combinations in the degenerate subspace are allowed. But the value of $\operatorname{Var}(G)$ remains unchanged under such redistributions, and we may still choose just two nonvanishing probabilities $p_{1}=$ $p_{L}=1 / 2$. The derivation did not make use of a multimode structure of the energy eigenstates. Hence, exactly the same minimal uncertainty of $\theta_{\text {est }}$ can be obtained by superposing the ground state of a single mode with a Fock state of given maximum allowed energy as with an arbitrarily entangled multimode state containing components of up to the same maximum energy. For a specific example, consider phase estimation in a Mach-Zehnder interferometer. It has $N=2$ modes, and a phase shift just in one of them, i.e., the relevant Hamiltonian is $H=\theta n_{1}$. Adding energy conservation of the two modes at the beam splitters (i.e., the fact that the accessible states are two-mode Fock states of the form $\left|n-n_{2}, n_{2}\right\rangle$, where $n_{2}$ with $0 \leq n_{2} \leq n$ is the number of photons in the second mode), one immediately finds that the optimal two-mode state is $(|n, 0\rangle+|0, n\rangle) / \sqrt{2}$, i.e., the highly entangled N00N state (Boto et al., 2000). However, we can achieve exactly the same variance of $G$ and hence sensitivity with the single-mode state $(|n\rangle+|0\rangle) / \sqrt{2} \otimes \rho_{2}$, i.e., a product state where we keep the second mode in any state $\rho_{2}$. In both cases the maximum energy of the first mode is $n \hbar \omega$ (assuming $\omega_{1}=\omega_{2}=\omega$ ), and the average energy in the interferometer $n \hbar \omega / 2$ (neglecting the vacuum energy $\hbar \omega / 2$ ). Hence, also from the perspective of maximum energy deposit in the optical components, there is no advantage in using two entangled modes. If the Mach-Zehnder interferometer is realized abstractly via Ramsey pulses on $N$ twolevel systems (states $|0\rangle,|1\rangle$ ) for the beam splitters, and a phase shift $\exp \left(i \theta J_{z}\right), J_{z}=\sum_{i=1}^{N} \sigma_{z}^{(i)} / 2$, the state that maximizes $\operatorname{Var}\left(J_{z}\right)$ is the (maximally entangled) GHZ state $(|0 \cdots 0\rangle+|1 \cdots 1\rangle) / \sqrt{2}$. But exactly the same uncertainty can be obtained with a single spin $j(j=N / 2)$ in the state $(|j, j\rangle+|j,-j\rangle) / \sqrt{2}$ (in the usual $|j, m\rangle$ notation, where $j$ is the total angular momentum and $m$ its $z$ component). Clearly, allowing as large a spectrum for a single system as for the combined systems makes entanglement entirely unnecessary here.

These considerations teach us that the relevant quantity to be maximized is the quantum uncertainty of the generator $G$. This can be understood in terms of generalized Heisenberg uncertainty relations, in which the generator $G$ plays the quantity complementary to $\theta$ as was found early on (Braunstein, Caves, and Milburn, 1996). In a multicomponent system maximizing $\operatorname{Var}(G)$ may be achieved with highly entangled states, but if the spectral range of a single system admits the same $\operatorname{Var}(G)$, there is no need for entanglement. If unbound spectra are permitted, one can in fact do much better than the Heisenberg limit: Berry et al. (2015) pointed out that the single-mode state $(\sqrt{3} / 2) \sum_{n=0}^{\infty} 2^{-n}\left|2^{n}\right\rangle$ has diverging $\operatorname{Var}(n)$ at the same time finite $\bar{n}$. It therefore allows, at least in principle and in an ideal setting, arbitrarily precise phase measurements while using finite energy.

Braun $(2011,2012)$ found a state of the form $(|0\rangle+$ $|2 n\rangle) / \sqrt{2}$ to be optimal for mass measurements with a nanomechanical oscillator given a maximum allowed number of excitation quanta $2 n$ and times much larger than the oscillation period (for shorter times there are contributions also from the dependence on frequency of the energy eigenfunctions). The same state of a single mode of the e.m. field is optimal for measuring the speed of light (Braun, Schneiter, and Fischer, 2017). In both cases the quantum uncertainties scale as $1 / n$ (quantum Fisher information proportional to $n^{2}$ ), and obviously no entanglement is needed. Of course, a state of the form $(|0\rangle+|2 n\rangle) / \sqrt{2}$ [called "half a N00N" state by Braun (2011)] is still highly nonclassical [see also De Pasquale et al. (2015)]. In fact, a single Fock state $|n\rangle$ is highly nonclassical as is witnessed by its highly oscillatory Wigner function (Schleich, 2001) with substantial negative parts. The superposition $(|0\rangle+|2 n\rangle) / \sqrt{2}$ leads in addition to $2 n$ lobes in the azimuthal direction that explain the sensitivity of phase measurements $\propto n^{-1}$. Alternatively, one can use superpositions of coherent states (Braginsky, Khalili, and Thorne, 1995; Lund et al., 2004; Neergaard-Nielsen et al., 2006; Suzuki et al., 2006; Wakui et al., 2007; Bimbard et al., 2010; Yukawa et al., 2013), i.e., "Schrödinger-cat" type states of the form $(|\alpha\rangle+|-\alpha\rangle) / \sqrt{2}$. They have been created in quantum optics with values of $\alpha=0.79$ in Ourjoumtsev et al. (2006). Lund et al. (2004) proposed a "breeding method" based on weak squeezing, beam mixing with an auxiliary coherent field, and photon detecting with threshold detectors to achieve values up to $\alpha \leq 2.5$, but the success probability was found to be too low for a realistic iterated protocol. An alternative based on homodyning was proposed by Laghaout et al. (2013) and Etesse et al. (2014) and implemented by Etesse et al. (2015), leading to $\alpha \simeq 1.63$. The current record in the optical domain for "large" $\alpha$ appears to be $\alpha \simeq \sqrt{3}$, achieved from two-mode squeezed vacuum and $n$-photon detection on one of the modes (Huang et al., 2015). In the microwave regime, superpositions of coherent states with $\alpha=$ $\sqrt{7}$ have been generated, as well as superposition of coherent states with smaller phase differences with up to 111 photons (Vlastakis et al., 2013). Monroe et al. (1996) reported superpositions of coherent states of the vibrational motion 
of a ${ }^{9} \mathrm{Be}^{+}$ion in a one-dimensional trap with $\alpha \simeq 3$. Almost arbitrary superpositions with a small number of photons can be generated by using couplings of a mode with two-level systems that can be tuned in and out of resonance, and a plethora of methods for generating superpositions of coherent states were proposed, but reviewing the entire literature of nonclassical states in general and even all the proposals for generating superpositions of coherent states is beyond the scope of the present review [see, e.g., Gottesman, Kitaev, and Preskill (2001), Deléglise et al. (2008), and Hofheinz et al. (2009) and the Nobel Lectures of Haroche (2013) and Wineland (2013) for historical accounts of the development of these fields and many more references, as well as the literature citing Montina and Arecchi (1998) where superpositions of coherent states in a Mach-Zehnder interferometer were studied with respect to the limitations arising from imperfect photodetectors].

The use of superpositions of coherent states for metrology was examined by Ralph (2002) and Gilchrist et al. (2004) and it was found that the Heisenberg limit can be reached. Montina and Arecchi (1998) discovered that superpositions of coherent states in atom interferometers may even exhibit quantumenhanced sensitivity to parameters that have no classical analog. For example, Riedel (2015) showed that monitoring the decoherence rate of a superposition of atomic coherent states may uncover clues about so-far undetected particles that couple softly (i.e., via weak momentum transfer, but not weakly) to the atoms. This is reminiscent of previous ideas of using decoherence as a sensitive probe (Braun and Martin, 2011). The decoherence rate can be detected with sensitivity that is limited only by the spatial size of the superposition, and the situation is quite similar to the estimation of boson loss discussed in Sec. II.E.

\section{Decoherence-enhanced measurements}

Decoherence is arguably the most fundamental issue that plagues quantum enhancements of all kinds, and quantumenhanced measurements are no exception. However, decoherence has interesting physical properties which imply that it can also be useful for precision measurements. This goes beyond the benefits of decoherence and open system dynamics found as early as the late 1990s and the early 2000s, when it was realized that entanglement can be created through decay processes or more generally through coupling to common environments (Plenio et al., 1999; Braun, 2002, 2005; Benatti, Floreanini, and Piani, 2003; Benatti, Liguori, and Nagy, 2008; Benatti, Floreanini, and Marzolino, 2009, 2010b), and, paradoxically, that decoherence of quantum computations can be reduced by rather strong dissipation that confines the computation to a decoherence-free subspace through a Zeno-type effect due to the rapid decay of states outside the decoherence-free subspace (Beige, Braun, and Knight, 2000; Beige et al., 2000). Recently such ideas have found renewed interest, and meanwhile techniques have been proposed to create steady state entanglement in driven open quantum systems, such as cold Rydberg gases in the Rydbergblockade regime (Lee, Cho, and Choi, 2015). It remains to be seen whether such stabilized entangled states are useful for precision measurements.
Here, on the contrary, we focus on the dynamics created by decoherence processes themselves. Decoherence arises from an interaction with an environment described by a nontrivial interaction Hamiltonian $H_{\text {int }}=\sum_{i} S_{i} B_{i}$ with a similar structure as the nonlinear Hamiltonians considered, where in $H_{\text {int }}$, however, one distinguishes operators $\left(S_{i}\right)$ pertaining to the system and others $\left(B_{i}\right)$ pertaining to the environment. The environment is typically considered as a heat bath with a large number of degrees of freedom that may not be entirely accessible (Weiss, 1999; Breuer and Petruccione, 2002; Benatti and Floreanini, 2005). In addition, a system and a heat bath have their own Hamiltonian, such that the total Hamiltonian reads $H=H_{S}+H_{B}+H_{\text {int }}$. From simple model systems it is known that decoherence tends to become extremely fast for quantum superpositions of states that differ macroscopically in terms of the eigenvalues of one of the $S_{i}$. For example, it was shown that superposing two Gaussian wave packets of a free particle in one dimension that is coupled through its position to a heat bath of harmonic oscillators leads to decoherence times that scale as powers of $\hbar$ that depend on how the wave packets are localized in phase space: The shortest decoherence time scaling as $\hbar / \mid q_{1}-$ $q_{2} \mid$ results from wave packets distinguished only by positions $q_{1}, q_{2}$, the longest one $\sim \hbar^{1 / 2} / \sqrt{\left|p_{1}-p_{2}\right|}$ from wave packets that differ only in their mean momenta $p_{1}, p_{2}$, and an intermediate one scaling as $\hbar^{2 / 3} /\left|\left(q_{1}-q_{2}\right)\left(p_{1}-p_{2}\right)\right|^{1 / 3}$. For systems of finite Hilbert-space dimensions such as angular momenta, one can often identify an effective $\hbar$ that scales as the inverse Hilbert-space dimension which suggests that monitoring the decoherence process can lead to highly sensitive measurements, possibly surpassing the $1 / \sqrt{N}$ scaling of the standard quantum limit.

That this intuition is correct was shown by Braun and Martin (2011), where a method was proposed for measuring the length of a cavity by monitoring the decoherence process of $N$ atoms inside the cavity. The atoms are initially prepared in a highly excited dark state, in which destructive interference prevents them from transferring their energy to a mode of the cavity with which they are resonant. For example, if one has two atoms coupling via an interaction $\left(g_{1} \sigma_{-}^{(1)}+g_{2} \sigma_{-}^{(2)}\right)^{\dagger}+$ H.c. to a mode of the cavity with annihilation operator $a$, a state $\propto\left(1 / g_{1}\right)|10\rangle-\left(1 / g_{2}\right)|01\rangle$ of the atoms (where $|0\rangle$ and $|1\rangle$ are the ground and excited states of the atoms) is a dark state, also known as a decoherence-free state: the amplitudes of photon transfer from the two atoms to the cavity cancel. However, the couplings $g_{i}$ depend on the position of the atoms relative to the cavity due to the envelope of the e.m. field. If the cavity changes its length $L$ with the atoms at fixed positions, the $g_{i}$ change, such that the original state becomes slightly bright. This manifests itself through the transfer of atomic excitations to the mode of the cavity, from where photons can escape and be detected outside. Braun and Martin (2011) showed that through this procedure the minimal uncertainty with which $L$ can be estimated (according to the quantum Cramér-Rao bound) scales as $1 / N$ even when using an initial product state of $N / 2$ pairs of atoms. This scaling applies both for a perfect cavity and in the bad cavity limit in which superradiance arises. Thus, at least in principle, Heisenberg-limited scaling can arise here without the need for 
an entangled state and in spite of the inherently decoherent nature of superradiance. However, the prefactor matters also here: Superradiance leads to a rapid decay of all states that are not dark, such that the available signal and with it the prefactor of the $1 / N$ scaling law deteriorates rapidly with time.

Given the delocalized nature of the cavity mode in this example, it is possible in principle to make the number of atoms that interact with the mode arbitrarily large, in contrast to the nonlinear schemes, for which the interactions have to decrease if the total energy is to remain an extensive quantity. But if the volume is kept fixed, the atoms will start to interact so that the simple independent atom model of superradiance breaks down. For even larger $N$ one has to increase the size of the cavity in order to accommodate all atoms. When the largest possible density is reached, the volume will have to grow $\propto N$, which implies coupling constants of the atoms to the cavity that decay as $1 / \sqrt{N}$ and leads back to standard quantum limit scaling. In addition, the number of atoms has to be macroscopic in order to compete with the best classical sensitivities reached with interferometers such as LIGO: Assuming that the prefactor in the $1 / N$ scaling is of the order of 1 , one needs $\sim 10^{21}$ atoms for a minimal uncertainty of $10^{-21}$. A cubic optical lattice with one atom every $\mu \mathrm{m}$, the lattice would have to span $10 \mathrm{~m}$ in the $x, y, z$ direction in order to accommodate that many atoms. When using a diamond with regularly arranged $\mathrm{N} V$ centers every $10 \mathrm{~nm}$ [such dense samples have been fabricated (Acosta et al., 2009)], one would still require a cubic diamond of edge length $10 \mathrm{~cm}$. These examples show that competing with the best classical techniques is very challenging even if one can achieve Heisenberg-limit scaling, as in classical protocols it is relatively easy to scale up the number of photons, compensating thus for a less favorable scaling with $N$.

\section{Coherent averaging}

There is nothing inherently quantum about the $1 / \sqrt{N}$ scaling of the standard quantum limit. Rather, this behavior is a simple consequence of the central limit theorem applied to $N$ independently acquired measurement results that are averaged as part of a classical noise-reduction procedure. The idea of coherent averaging is to replace the averaging by a coherent procedure, in which the $N$ probes all interact with a central quantum system (a "quantum bus"). In the end one measures the quantum bus or the entire system.

A simple example shows how this can lead to Heisenberglimit sensitivity without needing any entanglement: Consider $N$ spins $1 / 2$ interacting with a central spin $1 / 2$ with the Ising interaction $H_{\text {int }}=\sum_{i=1}^{N} g_{i} \sigma_{z}^{(0)} \otimes \sigma_{z}^{(i)}$, where the index zero indicates the central spin. The interaction commutes with the free Hamiltonian of all spins $H_{s}=\hbar \sum_{i=0}^{N} \omega_{i} \sigma_{z}^{(i)}$, and we can solve the time evolution exactly, starting from the initial product state

$$
|\psi(0)\rangle=\frac{1}{\sqrt{2}}\left(|0\rangle_{0}+|1\rangle_{0}\right) \otimes_{i=1}^{N}|0\rangle_{i}
$$

At time $t$, the state has evolved to

$$
|\psi(t)\rangle=\frac{1}{\sqrt{2}}\left(e^{i\left(\omega_{0} / 2-N \bar{g}\right) t}|0\rangle_{0}+e^{-i\left(\omega_{0} / 2-N \bar{g}\right) t}|1\rangle_{0}\right) \bigotimes_{i=1}^{N}|0\rangle_{i},
$$

up to an unimportant global phase factor. In particular, the state remains a product state at all times. If we measure $\sigma_{x}^{(0)}$ of the central spin, we find $\left\langle\sigma_{x}^{(0)}(t)\right\rangle=\cos \left[\left(\omega_{0}-2 N \bar{g}\right) t\right]$, i.e., the oscillation frequency increases for large $N$ proportional to $N$. As the quantum fluctuations of the central spin are independent of $N$, this implies a standard deviation in the estimation of the average coupling $\bar{g}$ that scales as $1 / N$, which can be confirmed by calculating the quantum Fisher information. Clearly, this is not an effect of entanglement, but simply of a phase accumulation. In this respect the approach is reminiscent of the sequential phase accumulation protocols in which the precision of a phase shift $\varphi$ measurement is enhanced by sending the light several times through the same phase shifter (Higgins et al., 2007). However, there the losses increase exponentially with the number of passes, and the sequential nature of the interaction leads to a bandwidth penalty that is absent for the simultaneous interaction described by $H_{\text {int }}$. Birchall et al. (2017) took into account the exponential loss of photons with the number of passes, and quantum Fisher information per scattered photon was optimized. It was found that when both probe and reference beam are subject to photon loss, the reduction of $\sigma\left(\varphi_{\text {est }}\right)$ by a nonclassical state compared to an optimal classical multipass strategy is only at most $\sim 19.5 \%$, and an optimal number of passes independent of the initial photon number was found, resulting in a loss of about $80 \%$ of all input photons. For a single-mode lossy phase the possible sensitivity gain is even smaller. Multipass microscopy was proposed by Juffmann, Klopfer et al. (2016) and it was experimentally demonstrated that at a constant number of photon sample interactions retardance and transmission measurements with a sensitivity beyond the single-pass shot-noise limit could be achieved. Similar ideas are being developed for electron microscopy (Juffmann, Koppell et al., 2016).

The fact that an interaction with $N$ probes and a central quantum bus can lead to Heisenberg-limit scaling of the sensitivity was first found by Braun and Martin (2011), where a more general model was studied. Note that the total Hamiltonian has exactly the same structure as for a decoherence model, with the $N$ probes playing the role of the original system, and the bus the role of an environment. However, in contrast to the standard scenario in decoherence, it is assumed here that at least part of the "environment" is accessible. This example shows that the environment can be as simple as a single spin $1 / 2$. One can also extend the model to include additional decoherence processes. Braun and Martin (2011) considered a phase-flip channel with rate $\Gamma$ on all spins. It was found that phase flips on any of the $N$ probes have no effect, whereas phase flip of the central spin introduces a prefactor that decays exponentially as $\exp (-2 \Gamma t)$ with time in $\left\langle\sigma_{x}^{(0)}(t)\right\rangle$. Since the prefactor is independent of $N$ the power-law scaling of the sensitivity with $N$ is unchanged, but it is clear that the prefactor matters and leads to a sensitivity that deteriorates exponentially with time.

The fact that the parameter estimated in the example is the interaction strength between the bus and the $N$ probes prevents a comparison with other schemes that do not use interactions. 
Fraïsse and Braun (2015) undertook a more comprehensive study of two different spin systems where also parameters describing the probes themselves and the bus were examined. Regimes of strong and weak interaction were analyzed, and different initial states considered. The two models, called $Z Z Z Z$ and $Z Z X X$ are, respectively, given by the Hamiltonians

$$
\begin{aligned}
& H_{1}=\frac{\omega_{0}}{2} \sigma_{z}^{(0)}+\frac{\omega_{1}}{2} \sum_{i=1}^{N} \sigma_{z}^{(i)}+\frac{g}{2} \sum_{i=1}^{N} \sigma_{z}^{(0)} \sigma_{z}^{(i)}, \\
& H_{2}=\frac{\omega_{0}}{2} \sigma_{z}^{(0)}+\frac{\omega_{1}}{2} \sum_{i=1}^{N} \sigma_{z}^{(i)}+\frac{g}{2} \sum_{i=1}^{N} \sigma_{x}^{(0)} \sigma_{x}^{(i)},
\end{aligned}
$$

where $\hbar=1$. The $Z Z Z Z$ model is an exactly solvable dephasing model; the $Z Z X X$ model can be analyzed numerically and with perturbation theory. The analysis is simplified when starting with a product state that is symmetric under exchange of the probes, in which case the probes can be considered as a single spin with total spin quantum number $j=N / 2$. It was found that for $\omega_{1}$, Heisenberg-limit scaling can be achieved in the $Z Z X X$ model when measuring the entire system, but not when measuring only the quantum bus, and not for the $Z Z Z Z$ model. Heisenberg-limit scaling for the uncertainty of $\omega_{0}$ is not possible in either model. For the interaction strength $g$, Heisenberg-limit scaling is found for a large set of initial states and range of interaction strengths when measuring the entire system, but only for a small set of initial states in the vicinity of the state considered in the simple example above, when measuring only the quantum bus. Interestingly, in the ZZZZ model Heisenberg-limit scaling of the sensitivity for measuring $g$ also arises with the probes in a thermal state at any finite temperature, as long as the quantum bus can be brought into an initially pure state. This is reminiscent of the power of 1 qubit (Knill and Laflamme, 1998): With a set of qubits of which only a single one is initially in a pure state, a quantum enhancement is already possible in quantum computation, providing evidence for an important role of quantum discord (Datta, Flammia, and Caves, 2005; Lanyon et al., 2008) (see Sec. II.C). As detailed in Sec. II.C, the DQC1 scheme can provide better-thanstandard quantum limit sensitivity as soon as the ancillas have a finite purity. The control qubit plays the role of the quantum bus, and the dipole-dipole interaction between the Rydberg atoms implements the $X X$ interaction considered by Fraïsse and Braun (2015).

An important limitation of such schemes was proven by Boixo et al. (2007) and Fraïsse and Braun (2016), where it was shown very generally that with a Hamiltonian extension to an ancilla system the sensitivity of a phase shift measurement cannot be improved beyond the best sensitivity achievable with the original system itself. Coherent averaging is nevertheless interesting as it allows one to achieve without injecting entanglement better-than-standard quantum limit sensitivity for which the noninteracting phase shift measurement would need a highly entangled state. Fraïsse and Braun (2017) showed that for general parameter-dependent Hamiltonians $H(\theta)$ the largest sensitivity is achieved if the eigenvectors of $(d / d \theta) H(\theta)$ to the largest and smallest eigenvalues are also eigenvectors of $H(\theta)$. If these eigenvalues are nondegenerate, the condition is also necessary. For a phase shift Hamiltonian the condition is obviously satisfied. This insight opens the way to Hamiltonian engineering techniques by adding parameter-independent parts to the Hamiltonian that remove or overwhelm parts that spoil the commutativity of $(d / d \theta) H(\theta)$ and $H(\theta)$ in the subspace of the largest and smallest eigenvalues of $(d / d \theta) H(\theta)$. These techniques were called "Hamiltonian subtraction" and "signal flooding," respectively, and were proposed to improve magnetometry with $\mathrm{N} V$ centers.

Another opportunity for Hamiltonian engineering arises if the eigenvalues of $H(\theta)$ do not depend on the parameter. Pang and Brun $(2014,2016)$ showed that in such a case the quantum Fisher information becomes periodic in time. This is particularly pernicious for quantum-enhanced measurement schemes that allow long measurement times, as under the condition of quantum coherence the quantum Fisher information typically increases quadratically with time if the eigenvalues of $H(\theta)$ depend on $\theta$. Adding another parameter-independent Hamiltonian might lead to parameter-dependent eigenvalues and hence unlock an unbound increase of the quantum Fisher information with time.

The existence of Heisenberg-limit scaling of sensitivities for product states suggests that coherent averaging might even be possible classically. This question was investigated by Braun and Popescu (2014) in a purely classical model of harmonic oscillators in which $N$ "probe" oscillators interact with a central oscillator. It was found that indeed for weak interaction strengths a regime of Heisenberg-limit scaling of the sensitivity exists, even though the scaling crosses over to standard quantum limit scaling for sufficiently large $N$. Nevertheless, it was proposed that this could be useful for measuring very weak interactions, and notably improve measurements of the gravitational constant.

\section{Quantum feedback schemes}

In the context of having probes interact with additional (ancilla) systems, quantum feedback schemes should be mentioned. This is an entire field by itself (D'Alessandro, 2007; Wiseman and Milburn, 2009; Serafini, 2012). Quantum feedback generalizes classical feedback loops to the quantum world: one tries to stabilize, or more generally dynamically control, a desired state of or an operation on a quantum system by obtaining information about its actual state or operation, and feeding back corrective actions into the controls of the system that bring it back to that desired state or operation if any deviation occurs. As a consequence, the field can be broadly classified according to two different categories: First, the object to be controlled may be a quantum state or an entire operation. And second, the type of information fed back can be classical or quantum. By "classical information" is meant information that is obtained from a measurement, and which is then typically processed on a classical computer and used to readjust the classical control knobs of the experiment. Such schemes are called "measurement-based feedback." In contrast to this, "coherent feedback" schemes directly use quantum systems that are then manipulated unitarily and fed back to the system.

Measurement-based feedback schemes in metrology are also known as "adaptive measurements" (see also Sec. V.D for 
adaptive measurements in the context of phase transitions). An adaptive scheme was proposed as early as 1988 by Nagaoka for mending the problem that the optimal POVM obtained in standard quantum parameter estimation depends on the a priori unknown parameter (Nagaoka, 1988). One starts with a random estimate, uses its value to determine the corresponding optimal POVM, measures the POVM, updates the estimate, uses that new value to determine a new optimal POVM, and so on. The scheme was shown to be strongly consistent (meaning unbiased for the number of iterations going to infinity) and asymptotically efficient (i.e., saturates the quantum Cramér-Rao bound in that limit) by Fujiwara (2006). It was experimentally implemented by Okamoto et al. (2012) as an adaptive quantum state estimation scheme for measuring polarization of a light beam, but is in principle a general purpose estimation scheme applicable to any quantum statistical model using identical copies of an unknown quantum state.

In the context of quantum optics, an adaptive homodyne scheme was proposed by Wiseman (1995) for measuring the phase of an optical mode in which the reference phase of the local oscillator $\Phi(t)$ is adjusted in real time to $\Phi(t) \simeq$ $\pi / 2+\varphi(t)$, where $\varphi(t)$ is the latest estimate of the phase carried by a continuous-wave, phase-squeezed light signal. This reference phase corresponds to the highest sensitivity in a homodyne scheme. Keeping the local oscillator phase through feedback close to this optimal operating point can beat nonadpative heterodyning in single shot phase decoding, as experimentally demonstrated by Armen et al. (2002). Pope, Wiseman, and Langford (2004) showed that adaptive measurements have a finite factor advantage even in the limit of arbitrarily weak coherent states. Phase estimation using feedback was also studied by Berry and Wiseman (2000, 2002, 2006) and Higgins et al. (2007). Berry and Wiseman (2002) investigated how well a stochastically varying, white noisecorrelated phase can be estimated. The theoretical analysis showed that the variance of the phase estimation could be reduced by a factor $\sqrt{2}$ by a simple adaptive scheme compared to a nonadaptive heterodyne scheme, resulting in the value of $n^{-1 / 2} / \sqrt{2}$, where $n$ is the number of photons per coherence time. With a squeezed beam and a more accurate feedback, the scaling can be improved to $n^{-2 / 3}$. The latter result was also found for a narrow-band squeezed beam (Berry and Wiseman, 2006, 2013). Yonezawa et al. (2012) reported a $15 \pm 4 \%$ reduction of a mean square error of the phase below the coherent-state limit with this scheme in optical-phase tracking, i.e., in a case without any a priori information about the value of the signal phase. The broad support of the signal phase implies that there is an optimal amount of squeezing, and the sensitivity enhancement is directly given by the squeezing. The scheme can therefore be seen as a generalization of Caves' idea of reducing the uncertainty with which a fixed phase shift in one arm of an interferometer can be measured (Caves, 1981). Instead of having a fixed phase reference by the beam in the other arm of the interferometer, the feedback allows one to continuously adjust the phase of the reference in the homodyning scheme to the optimal operating point. Clark, Stokes, and Beige (2016) proposed a feedback scheme based on measured temporal correlations ( $g^{(2)}$ correlation function) for estimating the phase of a coherent state inside a cavity and found that the uncertainty scales better than the standard quantum limit, namely, as $n^{-0.65}$, where $n$ is the mean photon number of the coherent state. Wheatley et al. (2010) experimentally used an "adaptive quantum smoothing method" for estimating a stochastically fluctuating phase on a coherent beam. "Smoothing" refers to the fact that estimates are obtained not only from data measured up to the time when one wants to estimate the phase, but also on later data. This implies, of course, that these smoothed estimates can be calculated only after a sufficient delay or at the end of the experiment, whereas feedback itself at time $t$ can use data only from times $t^{\prime} \leq t$ (or even $t^{\prime}<t$ when considering finite propagation times). Theory predicts a reduction of the mean square error by a factor $2 \sqrt{2}$ compared to the standard quantum limit (achievable by nonadaptive filtering, i.e., without feedback and using only previous data at any time), and an experimental reduction of about $2.24 \pm 0.14$ was achieved.

Quantum error correction for quantum-enhanced measurements, recently introduced by Dür et al. (2014) and Kessler et al. (2014), can also be seen in the context of quantum feedback schemes (Ahn, Doherty, and Landahl, 2002). Quantum error correction is one of the most important ingredients of quantum computing (Shor, 1995; Gottesman, 1996; Steane, 1996). The general idea, for both quantum computing and quantum-enhanced measurements, is that one wants to apply recovery operations $\mathcal{R}$ to a state that after encoding the desired information through an operation $\mathcal{M}$ has been corrupted by a noise process $\mathcal{E}$, such that $\mathcal{R} \circ \mathcal{E} \circ \mathcal{M}(\rho) \propto \mathcal{M} \rho$. Kessler et al. (2014) showed that this can be achieved for the sensing of a single qubit subject to dephasing noise if it is coupled to a pure ancilla bit. Syndrome measurements (i.e., measurements of collective observables which do not destroy the relevant phase information, a concept developed in quantum error correction) of both qubits at a rate faster than the dephasing rate allow one to detect whether a phase flip has occurred and to correct it, extending in this way the coherence time available for Ramsey interferometry to much longer times and thus better maximum sensitivities. When using $N$ qubits in parallel, an ancilla is not necessary. The method then operates directly on an initially entangled state, such as the GHZ state, and measures error syndromes on pairs of spins. Thus, the idea here is not so much to avoid entanglement but rather to stabilize through rapid multispin error-syndrome measurements the correct imprinting of the information on the quantum state against unwanted decoherence processes. For phase estimation on $N$ qubits evolving in parallel under individual and identical Pauli rankone noisy channels, fast control schemes based on quantum error correction allow one to restore the Heisenberg limit by completely eliminating the noise at the cost of slowing down the unitary evolution by a constant factor, unless the noise is dephasing noise that couples to the same Pauli operator as the Hamiltonian generating the phase shift (Sekatski et al., 2017).

More generally, one can prove that sequential metrological schemes involving an initial probe entangled with an ancilla, with the probe undergoing $N$ passes of a transformation encoding the parameter of interest, interspersed by arbitrary 
feedback control operations acting on probe and ancilla, and followed by a joint measurement on the two particles at the output, can outperform any parallel metrological scheme relying on an initial $N$-particle entangled state (DemkowiczDobrzański and Maccone, 2014; Yuan and Fung, 2015; Huang, Macchiavello, and Maccone, 2016; Yuan, 2016; Sekatski et al., 2017). In particular, Yuan (2016) showed that a sequential feedback scheme allows one to realize a joint quantum-enhanced measurement of all three components of a magnetic field on a single-qubit probe. As remarked in Sec. II.A, the use of sequential schemes assisted by suitable control reduces the input demand from multipartite to bipartite entanglement, resulting in a notable technological advantage.

\section{THERMODYNAMICAL AND NONEQUILIBRIUM STEADY STATES}

This section concerns precision parameter estimations when probes are thermal states or nonequilibrium steady states of dissipative dynamics. These states have the advantage to be stationary and describe mesoscopic systems. From measurements on these probes, intensive parameters, such as temperature, chemical potential, or couplings of Hamiltonians or of dissipators, are inferred with a sensitivity given by the inverse of the quantum Fisher information. Thermal probes are crucial for both fundamental issues and technological applications (Benedict, 1984; Childs, 2001; Giazotto et al., 2006). Estimations with dissipative dynamics (Bellomo et al., 2009, 2010a, 2010b; Alipour, Mehboudi, and Rezakhani, 2014; Zhang and Sarovar, 2015) are also instances of process tomography (Mohseni, Rezakhani, and Lidar, 2008; Bendersky and Paz, 2013; Merkel et al., 2013; Baldwin, Kalev, and Deutsch, 2014) with partial prior knowledge. The identification of the quantum Fisher information with the Bures metric clarifies the role of criticality as a resource for estimation sensitivity. With extensive, i.e., linear in the system size, interactions and away from critical behaviors, the Bures distance between the probe state and its infinitesimal perturbation is at most extensive. Critical behaviors, e.g., separation between different states of matter or long-range correlations, are thus characterized by a superextensive Bures metric and the quantum Fisher information. Thus, the next sections are dedicated to superextensivity of the quantum Fisher information as a signature of enhancements in precision measurements on thermodynamical and nonequilibrium steady states. Emphasis will be put on highly sensitive probes that do not need to be entangled and in certain cases not even quantum.

\section{A. Thermodynamical states and thermal phase transitions}

Thermodynamic states at equilibrium are derived by the maximization of information-theoretic Shannon's entropy (Jaynes, 1957a, 1957b), equivalent to the maximization of the number of microscopic configurations compatible with physical constraints. Given the probability distribution $\left\{p_{j}\right\}_{j}$ of a set of configurations $\{j\}_{j}$, the constraints are the normalization $\sum_{j} p_{j}=1$ and the averages of certain quantities $\left\langle F^{(k)}\right\rangle=\sum_{j} p_{j} f_{j}^{(k)}, f_{j}^{(k)}$ being the values of the quantity
$F^{(k)}$ corresponding to the $j$ th configuration. The solution of the maximization is the well-known Boltzmann-Gibbs distribution

$$
p_{j}=\frac{e^{-\sum_{k} \theta_{k} f_{j}^{(k)}}}{Z}, \quad Z=\sum_{j} e^{-\sum_{k} \theta_{k} f_{j}^{(k)}}
$$

where $Z$ is the partition function, and $\theta_{k}$ is the Lagrange multiplier corresponding to the quantity $F^{(k)}$ fixed on average.

This formalism is equally adequate for both classical and quantum thermodynamic systems. In the quantum case all the quantities $F^{(k)}$ are commuting operators, the configurations are labeled by the set of eigenvalues of these operators and possibly additional quantum numbers in the case of degeneracy, and the thermal state is the density matrix $\rho$ diagonal in the common eigenbasis of the $F^{(k)}$ with eigenvalues $p_{j}$ :

$$
\rho=\sum_{j} p_{j}|j\rangle\left\langle j\left|, \quad F^{(k)}\right| j\right\rangle=f_{j}^{(k)}|j\rangle .
$$

Lagrange multipliers are the thermodynamic parameters to be estimated. Since the density matrix in Eq. (83) depends on them only through its eigenvalues $p_{j}$, the quantum Fisher matrix $I=\left[I_{\theta_{k}, \theta_{k^{\prime}}}\right]_{k, k^{\prime}}$ coincides with the classical Fisher matrix of the probability distribution $\left\{p_{j}\right\}_{j}$. A straightforward computation shows

$$
I_{\theta_{k}, \theta_{k^{\prime}}}=\frac{\partial^{2} \ln Z}{\partial \theta_{k} \partial \theta_{k^{\prime}}}=\operatorname{Cov}\left(F^{(k)}, F^{\left(k^{\prime}\right)}\right)
$$

See also Jiang (2014) for the computation of the quantum Fisher information with density matrices in exponential form. The diagonal element $I_{\theta_{k}, \theta_{k}}$ is the largest inverse sensitivity for a single estimation of the parameter $\theta_{k}$, while the Fisher matrix $I$ bounds the inverse covariance matrix of the multiparameter estimation; see Eq. (17). The Cramér-Rao bound hence reads

$$
\left[\operatorname{Cov}\left(\theta_{k, \text { est }}, \theta_{k^{\prime}, \text { est }}\right)\right]\left[\operatorname{Cov}\left(F^{(k)}, F^{\left(k^{\prime}\right)}\right)\right] \geq \frac{1}{M},
$$

which is the uncertainty relation for conjugate variables in statistical mechanics (Gilmore, 1985; Davis and Gutiérrez, 2012).

The computation of the Fisher matrix (84), together with the Cramér-Rao bound (85), implies that the best sensitivity of Lagrange multipliers $\left\{\theta_{k}\right\}_{k}$ is inversely proportional to squared thermal fluctuations and thus susceptibilities; see, e.g., Reichl (1998). For connections among the metric of thermal states, Fisher information, and susceptibilities see Weinhold (1975), Ruppeiner (1979, 1981, 1991, 1995), Diósi et al. (1984), Mrugała (1984), Salamon, Nulton, and Ihrig (1984), Nulton and Salamon (1985), Janyszek (1986b, 1990), Janyszek and Mrugała (1989), Brody and Rivier (1995), Dolan (1998), Janke, Johnston, and Malmini (2002), Brody and Ritz (2003), Janke, Johnston, and Kenna (2003), Crooks (2007), Prokopenko et al. (2011), and Davis and Gutiérrez (2012) for classical systems and Janyszek (1986a), Janyszek and Mrugała (1990), You, Li, and Gu (2007), Zanardi, 
Campos Venuti, and Giorda (2007), Paunković and Vieira (2008), Zanardi, Paris, and Campos Venuti (2008), Quan and Cucchietti (2009), and Marzolino and Braun (2013, 2015) for quantum systems.

Because of the pairwise commutativity of the $F^{(k)}$, the estimations of the parameters $\left\{\theta_{k}\right\}_{k}$ are reduced to parameter estimations with the classical probability distribution $\left\{p_{j}\right\}_{j}$. Thus, the maximum-likelihood estimator is asymptotically unbiased and optimal, in the sense of achieving the CramérRao bound, in the limit of infinitely many measurements (Helstrom, 1976; Holevo, 1982). This estimator consists of measuring each $F^{(k)}$ with outcomes $\left\{f_{j}^{(k)}\right\}_{j=1, \ldots, M}$, and in maximizing the average logarithmic likelihood $\ell=$ $(1 / M) \ln \prod_{j} p_{j}$ with respect to the parameters $\left\{\theta_{k}\right\}_{k}$.

Among the most common statistical ensembles for equilibrium systems there are the canonical ensemble and the grand canonical ensemble. The canonical ensemble describes systems that exchange energy only with their surroundings: the only quantity $F_{1}=H$ fixed on average is the Hamiltonian, the Lagrange multiplier is $\theta_{1}=\beta=1 / k_{B} T$ where $k_{B}$ is the Boltzmann constant and $T$ is the absolute temperature, and the Fisher information is proportional to the heat capacity $C_{V}=(\partial\langle H\rangle / \partial T)_{V}$,

$$
I_{\beta, \beta}=\operatorname{Var}(H)=k_{B} T^{2} C_{V} .
$$

The grand canonical ensemble describes systems that exchange energy and particles with the surrounding: the quantities fixed on average are the Hamiltonian $F_{1}=H$ and the particle number $F_{2}=N$, with Lagrange multipliers being the inverse temperature $\theta_{1}=\beta=1 / k_{B} T$ and $\theta_{2}=-\beta \mu$ where $\mu$ is the chemical potential. The Fisher information of temperature and the chemical potential are linked to thermal fluctuations, i.e., heat capacity $C_{V}=(\partial\langle H\rangle / \partial T)_{V}$ and isothermal compressibility $\kappa_{T}=-(1 / V)(\partial V / \partial P)_{T}$, respectively, where $V$ is the volume and $P$ is the pressure (Marzolino and Braun, 2013, Marzolino and Braun, 2015):

$$
\begin{gathered}
I_{\beta, \beta}=\operatorname{Var}(\mu N-H)=\frac{\partial \mu\langle N\rangle}{\partial \beta}+k_{B} T^{2} C_{V}, \\
I_{\mu, \mu}=\beta^{2} \operatorname{Var}(N)=\frac{\langle N\rangle^{2}}{\beta V} \kappa_{T} .
\end{gathered}
$$

Another parameter that can be estimated within this framework is the magnetic field. For certain classical magnetic or spin systems, the interaction with a magnetic field $\boldsymbol{B}$ is $\boldsymbol{B} \cdot \boldsymbol{M}$, with $\boldsymbol{M}$ being the total magnetization. This interaction term can represent a contribution to the Hamiltonian as well as additional "fixed-on-average quantities" $\boldsymbol{B}$ with Lagrange multipliers $\beta \boldsymbol{M}$. The magnetic field is also linked to magnetic susceptibility $\chi=\partial\langle M\rangle / \partial B$ (where for simplicity $\boldsymbol{B}$ and $\boldsymbol{M}$ have only a single component, $M \equiv M_{z}, B \equiv B z$ ):

$$
I_{B, B}=\beta^{2} \operatorname{Var}(M)=\beta \chi .
$$

This picture of the magnetic field as a Lagrange multiplier is valid also for coupling constants whenever the Hamiltonian is $H=\sum_{j} \lambda_{j} H_{j}$, where $\beta \lambda_{j}$ is the Lagrange multiplier of $H_{j}$. For general quantum systems, the noncommutativity of magnetization or other Hamiltonian contributions $H_{j}$ with the rest of the Hamiltonians gives rise to quantum phase transitions that occur also at zero temperature without thermal fluctuations. These considerations also apply to the so-called generalized Gibbs ensembles, i.e., with arbitrary fixed-on-average quantities $F^{(k)}$, for which estimations of parameters $\theta_{k}$ are under experimental (Langen et al., 2015) and theoretical (Foini et al., 2017) study.

Thermal susceptibilities are typically extensive except in the presence of phase transitions. Thus, their connection with Fisher information suggests that thermal states at critical points (Baxter, 1982; Diósi et al., 1984; Janyszek and Mrugała, 1989; Janyszek, 1990; Ruppeiner, 1991, 1995; Brody and Rivier, 1995; Dolan, 1998; Reichl, 1998; Janke, Johnston, and Malmini, 2002; Brody and Ritz, 2003; Janke, Johnston, and Kenna, 2003; Prokopenko et al., 2011) with divergent susceptibilities are probes for enhanced measurements. Thermal susceptibility divergences occur also in classical systems, proving precision measurements without entanglement.

\section{Role of quantum statistics}

The estimation of Lagrange multipliers of quantum gases in the grandcanonical ensemble and the role of quantum statistics therein (Marzolino and Braun, 2013, 2015) are now discussed. Consider ideal gases in a homogeneous or harmonic trap. $I_{\beta, \beta}$ is always extensive in the average particle number $\langle N\rangle$. The corresponding relative error found in Marzolino and Braun $(2013,2015)$ for temperature estimation is still 1 order of magnitude smaller than the standard deviations obtained experimentally via density measurements of bosons (Leanhardt et al., 2003) and fermions (Müller et al., 2010; Sanner et al., 2010).

Estimations of chemical potentials are more sensitive to quantum statistics than estimations of temperature, because the chemical potential is the conjugate Lagrange multiplier of the particle number which in turn reveals clear signatures of quantum statistics, such as bunching and antibunching. Effects of quantum statistics are more evident in quantum degenerate gases, i.e., at low temperatures.

In fermion gases, $I_{\mu, \mu}$ is extensive but diverges at zero temperature. A change in the chemical potential corresponds to the addition or the subtraction of particles, thus achieving a state orthogonal to the previous one. This sudden state change makes the chemical potential estimation very sensitive. A generalization of the Cramér-Rao bound called the Hammerseley-Chapman-Robbins-Kshirsagar bound that is suitable for nondifferentiable statistical models (Tsuda and Matsumoto, 2005) must be applied. This may lead to superextensive $I_{\mu, \mu}$ depending on the degree of rotational symmetry breaking or confinement anisotropy and dimension [see Appendix B in Marzolino and Braun (2013)].

Bose gases undergo a phase transition to a Bose-Einstein condensate in three dimensions for homogeneous confinement, and in two or three dimensions in a harmonic trap. Approaching from above the critical temperature, or zero temperature with large density when there is no phase transition, $I_{\mu, \mu}$ is superextensive: for homogeneous and harmonic traps, respectively, 


$$
\begin{gathered}
I_{\mu, \mu} \lesssim \begin{cases}\mathcal{O}\left(\beta^{2}\langle N\rangle^{4 / 3}\right) & \text { in three dimensions, } \\
\mathcal{O}\left(\beta^{2} \frac{\langle N\rangle^{2}}{\log \langle N\rangle}\right) & \text { in two dimensions, } \\
\mathcal{O}\left(\beta^{2}\langle N\rangle^{2}\right) & \text { in one dimension, }\end{cases} \\
I_{\mu, \mu} \lesssim \begin{cases}\mathcal{O}\left(\beta^{2}\langle N\rangle\right) & \text { in three dimensions, } \\
\mathcal{O}\left(\beta^{2}\langle N\rangle \log \langle N\rangle\right) & \text { in two dimensions, } \\
\mathcal{O}\left(\beta^{2} \frac{\langle N\rangle^{2}}{\log \langle N\rangle}\right) & \text { in one dimension. }\end{cases}
\end{gathered}
$$

Below the critical temperature, the Bose-Einstein condensate phase depends on the anisotropy of the external potential. If the gas is much less confined along certain directions, the Bose-Einstein condensate is an effective low-dimensional gas with excitations restricted to directions along the less confined dimensions. A hierarchy of condensations to subsequent lower-dimensional gases is possible. These Bose-Einstein condensates have been studied both at finite size and in the thermodynamic limit focusing on mathematical structures and general properties (Girardeau, 1960, 1965; Casimir, 1968; Krueger, 1968; Rehr, 1970; van den Berg and Lewis, 1982; van den Berg, 1983; van den Berg, Lewis, and Munn, 1986; van den Berg, Lewis, and Pulé, 1986; Ketterle and van Druten, 1996; Mullin, 1997; van Druten and Ketterle, 1997; Zobay and Garraway, 2004; Beau and Zagrebnov, 2010; Mullin and Sakhel, 2012), in connection with liquid helium in thin films (Osborne, 1949; Mills, 1964; Douglass, Khorana, and Brij, 1965; Goble and Trainor, 1965, 1966, 1967; Khorana and Douglass, 1965), magnetic flux of superconducting rings (Sonin, 1969), and gravito-optical traps (Wallis, 1996). Experimental realizations of effective low-dimensional Bose-Einstein condensates with trapped atoms have been reported by Görlitz et al. (2001), Greiner et al. (2001), Esteve et al. (2006), van Amerongen (2008), van Amerongen et al. (2008), Armijo et al. (2011), and Bouchoule, van Druten, and Westbrook (2011). $I_{\mu, \mu}$ in these Bose-Einstein condensate phases is superextensive and interpolates between the scaling above the critical temperature and $I_{\mu, \mu}=\mathcal{O}\left(\beta^{2}\langle N\rangle^{2}\right)$ for a standard Bose-Einstein condensate consisting only of the ground state. The advantage of BoseEinstein condensate probes for precision estimations is that $I_{\mu, \mu}$ is superextensive in the entire Bose-Einstein condensate phase and not only at critical points as for susceptibilities of other thermal phase transition.

In a mean field model with interactions treated perturbatively, if the ideal system exhibits a superlinear scaling of the Fisher information, the interaction strength $\lambda$ has to go to zero for $N \rightarrow \infty$ for the perturbation theory to remain valid. In this limit, the superlinear scaling disappears for any nonzero value of $\lambda$, but at finite $N$ there are values of $\lambda$ which do not destroy the sub-shot noise.

Moreover, superextensive quantum Fisher information in one dimension at fixed volume $L_{x}$ and small contact interaction coupling $c / L_{x}$ results

$$
\begin{aligned}
I_{\mu, \mu} \simeq & \frac{\beta^{2} \lambda_{T}^{2}\langle N\rangle^{3}}{2 \pi L_{x}^{2}}+\beta^{2}\langle N\rangle-\frac{\beta^{2} \lambda_{T}^{4}\langle N\rangle^{4}}{8 \pi^{2} L_{x}^{4}}\left(1-e^{-4 \pi / \lambda_{T}^{2} \rho^{2}\langle N\rangle}\right) \\
& +c\left[\frac{3 \beta^{3} \lambda_{T}^{6}\langle N\rangle^{7}}{16 \pi^{3} L_{x}^{7}}\left(1-e^{-4 \pi / \lambda_{T}^{2} \rho^{2}\langle N\rangle}\right)\right. \\
& \left.-\frac{\beta^{3} \lambda_{T}^{4}\langle N\rangle^{6}}{4 \pi^{2} L_{x}^{4}}\left(2+e^{-4 \pi / \lambda_{T}^{2} \rho^{2}\langle N\rangle}\right)\right]
\end{aligned}
$$

where $\lambda_{T}$ is the thermal wavelength, in agreement with experimental measurements on atom chips using ${ }^{87} \mathrm{Rb}$ atoms (Armijo et al., 2011). Superextensive grand canonical fluctuations of particle number, and thus superextensive $I_{\mu, \mu}$, have been observed in a photon Bose-Einstein condensate (Schmitt et al., 2014), which can be realized at room temperature (Klaers, 2014).

\section{Interferometric thermometry}

A protocol for precision thermometry proposed by Stace (2010) utilizes a Mach-Zehnder interferometer coupled to an ideal gas consisting of $N$ two-level atoms in the canonical ensemble. The gas Hamiltonian is $H_{0}=\sum_{j=1}^{N} \epsilon|\epsilon\rangle_{j}\langle\epsilon|$, where $|\epsilon\rangle_{j}$ is the $j$ th particle excited state with singleparticle energy $\epsilon$, while the single-particle lowest energy is zero. The label $j$ at the bra-vector in the projector is skipped for brevity.

The interferometer is injected with $K$ two-level atoms that interact with the gas in one arm of the interferometer with the interaction Hamiltonian $H_{I}=\alpha \sum_{j=1}^{K} \sum_{l=1}^{N}|\epsilon\rangle_{j}\langle\epsilon|\otimes| \epsilon\rangle_{l}\langle\epsilon|$, where the index $j$ labels the atoms in the interferometer and $l$ refers to the atoms in the gas. In order for the interaction not to sensitively perturb the gas, the interferometer should be much smaller than the gas, thus $K \ll N$. Each atom in the interferometers acquires a relative phase $\phi=\alpha m \tau$ between the arms, where $\tau$ is the interaction time and $m$ is the number of excited atoms in the gas whose expectation $\langle m\rangle=N /\left(1+e^{\beta \epsilon}\right)$ depends on the temperature.

The inverse temperature can be estimated from the interferometric phase measurement with the sensitivity

$$
\sigma(\beta)=\frac{\delta \phi}{|\alpha \tau(d\langle m\rangle / d \beta)|}=\frac{\left(1+e^{\beta \epsilon}\right)^{2}}{\epsilon e^{\beta \epsilon} N} \frac{\delta \phi}{\alpha \tau},
$$

resulting from error propagation, where $\delta \phi$ is the best sensitivity of the phase estimation according to the quantum Cramér-Rao bound. The scaling with the number $K$ of probes comes from $\delta \phi$. For distinguishable atoms, separable states imply shot noise $\delta \phi=1 / K^{1 / 2}$, while sub-shot noise can be achieved with separable states of identical atoms as discussed in Sec. III, leading to $\delta \beta \propto 1 / K$ for ideal noninteracting bosons, or $\delta \beta \propto 1 / K^{p+1 / 2}$ for ideal fermions with a dispersion relation of the probe atoms as discussed after Eq. (67). Note also that the scaling of the sensitivity (93) with respect to the particle number $N$ in the gas looks like a Heisenberg scaling irrespectively of the probe state, and it is not possible to achieve this scaling by direct measurement of the gas (i.e., without any probe) because the Fisher information is extensive except at critical points. What is conventionally considered is, 
however, the scaling with the number of probes, in this case $K$, which can be controlled.

Optimal thermometry with a single quantum probe (and hence by definition without entanglement) was discussed by Correa et al. (2015). It was found that the quantum CramérRao bound for $T$ of a thermalizing probe reproduces the wellknown relation of temperature fluctuations to specific heat $C_{V}(T),\left[T / \operatorname{Var}\left(T_{\text {est }}\right)\right]^{2} \leq C_{V}(T)$ [with Boltzmann constant $k_{B}=1$, see also Jahnke, Lanéry, and Mahler (2011)]. The level structure of the probe was then optimized to obtain maximum heat capacity and it was found that the probe should have only two different energy levels, with the highest one maximally degenerate and a nontrivial dependence of the optimal energy gap on temperature (Reeb and Wolf, 2015). The sensitivity of the probe increases with the number of levels, but the role of the quantumness of the initial state of the probe on the sensitivity of thermometry is not fully understood yet. Interferometric thermometry with a single probe was realized experimentally in an NMR setup in Raitz et al. (2015) and the role of quantum coherence emphasized. Experimental simulations in quantum optical setups and investigations of the role of quantum coherence were reported by Mancino et al. (2016) and Tham et al. (2016). The theoretical study by Jevtic et al. (2015) examined thermometry with two qubits. Numerical evidence suggested that while initial quantum coherences can improve the sensitivity, the optimal initial state is not maximally entangled.

\section{B. Thermodynamical states and quantum phase transitions}

Quantum phase transitions are sudden changes of the ground state for varying Hamiltonian parameters. If the Hamiltonian $H(\boldsymbol{\theta})=\sum_{j \geq 0} E_{j}(\boldsymbol{\theta})\left|E_{j}(\boldsymbol{\theta})\right\rangle\left\langle E_{j}(\boldsymbol{\theta})\right|$ with $E_{j} \leq$ $E_{j+1}$ has a unique pure ground state $\left|E_{0}(\boldsymbol{\theta})\right\rangle$, the Fisher matrix (Campos Venuti and Zanardi, 2007; You, Li, and Gu, 2007; Zanardi, Giorda, and Cozzini, 2007; Gu and Lin, 2009) is

$$
I_{\theta_{k}, \theta_{k^{\prime}}}=4 \operatorname{Re} \sum_{j>0} \frac{\left\langle E_{0}\left|\partial_{\theta_{k}} H\right| E_{j}\right\rangle\left\langle E_{j}\left|\partial_{\theta_{k^{\prime}}} H\right| E_{0}\right\rangle}{\left(E_{j}-E_{0}\right)^{2}}
$$

which follows from the differentiation of the eigenvalue equation $H\left|E_{j}\right\rangle=E_{j}\left|E_{j}\right\rangle$ or from the standard timeindependent perturbation theory with respect to small variations $d \boldsymbol{\theta}$. The quantum Fisher information is also expressed in terms of the imaginary time correlation function or dynamical response function (Campos Venuti and Zanardi, 2007; You, Li, and Gu, 2007; Gu and Lin, 2009; You and He, 2015) and has been used to count avoided crossings (Wimberger, 2016).

Equation (94) tells us that the Fisher matrix can diverge only for divergent Hamiltonian derivatives or for gapless systems $E_{1}-E_{0} \rightarrow 0$ in the thermodynamic limit. Thus, the divergence or the superextensivity of the quantum Fisher information reveals a quantum phase transition but the converse does not hold; see Gu (2010) for a review. Finite size scaling of the quantum Fisher information (Campos Venuti and Zanardi, 2007; Zanardi, Paris, and Campos Venuti, 2008; Gu and Lin, 2009) can be derived using finite size scaling at criticality (Brankov, Danchev, and Tonchev, 2000; Continentino, 2001).

Superextensive quantum Fisher information was observed at low order symmetry breaking quantum phase transitions, topological quantum phase transitions, and gapless phases, but this criterion may fail at high-order symmetry breaking quantum phase transitions (Tzeng et al., 2008) and Berezinskii-Kosterlitz-Thouless (BKT) quantum phase transitions (Chen et al., 2008; Sun, Kolezhuk, and Vekua, 2015). The following sections focus on quantum critical systems at zero temperature exhibiting superextensive quantum Fisher information of Hamiltonian parameters without entanglement. The notation of existing literature is adapted and unified by writing down a general parametrized Hamiltonian whose different special cases are studied in the literature.

\section{Quasifree fermion models}

Consider noninteracting many-body Hamiltonians, i.e.,

$$
H(\boldsymbol{\theta})=\sum_{j} \omega_{j}(\boldsymbol{\theta}) a_{j}^{\dagger}(\boldsymbol{\theta}) a_{j}(\boldsymbol{\theta}),
$$

where $a_{j}^{\dagger}\left(a_{j}\right)$ creates (annihilates) a fermion in the $j$ th eigenmode. The dependence of the eigenmodes $a_{j}^{\dagger}, a_{j}$ on the parameters corresponds in second quantization to the dependence of the Hamiltonian eigenvectors on $\boldsymbol{\theta}$, as required for the ground state to be sensitive to variations of $\boldsymbol{\theta}$. The eigenstates of Eq. (95), and thus thermal probes including the ground state, are not entangled in the eigenmodes. Therefore, measurements on the ground state provide parameter estimation without entanglement and with enhanced precision at phase transitions, as shown by the following examples.

Under Bogoliubov transformations the Hamiltonian (95) can be mapped into many models studied in the literature. Bogoliubov transformations preserve neither mode entanglement nor operation locality, as discussed in Sec. III. The relativity of entanglement with respect to the basis of modes provides complementary pictures of the Hamiltonian and of estimation protocols of its parameters but does not change the physics: either the probe state is entangled in one basis or it is not in another and the enhanced estimation precision is achieved by nonlocal measurements as a consequence of long-range correlations at criticality. This situation is reminiscent of the case of interferometry with identical particles discussed in Sec. III, for which rotations of modes redistribute quantum resources between initial entanglement and nonlocal interferometers. Moreover, Bogolibov transformations and corresponding rotated modes are experimentally addressed in several physical systems (Segovia et al., 1999; Vogels et al., 2002; Moritz et al., 2003; Davis et al., 2006; Robillard et al., 2008; Sattler, 2011; Inguscio and Fallani, 2013; Hu, Xianlong, and Liu, 2014; Yan, Huang, and Wang, 2016), showing that quasiparticles represent legitimate and experimentally relevant subsystems.

Hamiltonians equivalent to Eq. (95) under Bogoliubov transformations are quasifree fermion models (Cozzini, Giorda, and Zanardi, 2007), i.e., quadratic Hamiltonians in the creation $c_{j}^{\dagger}$ and annihilation $c_{j}$ operators of $L$ fermionic modes: 


$$
H_{\text {quasifree }}=\sum_{j, l=1}^{L}\left(c_{j}^{\dagger} A_{j, l} c_{l}+\frac{1}{2}\left(c_{j}^{\dagger} B_{j, l} c_{l}^{\dagger}+\text { H.c. }\right)\right)
$$

where $L$ is a measure of the system volume. Translationally invariant Hamiltonians (96) with periodic boundary conditions and tunneling in the modes $\left\{c_{j}, c_{j}^{\dagger}\right\}_{j}$ of range $r$ have

$$
\begin{aligned}
& A_{j, l}=(J-\mu) \delta_{j, l}-J \theta(r-|j-l|)-J \theta(|j-l|-L+r), \\
& B_{j, l}=J \gamma \operatorname{sgn}(j-l)[\theta(r-|j-l|)-\theta(|j-l|-L+r)],
\end{aligned}
$$

where $J>0, \operatorname{sgn}(0)=0$, and $\theta(\cdot)$ is the unit step function with $\theta(0)=1$. Here $J$ corresponds to a tunneling energy between different sites, and $J \gamma$ to an effective interaction; $\mu$ multiplies the total particle number and hence corresponds to a chemical potential. This Hamiltonian can be analytically diagonalized (Cozzini, Giorda, and Zanardi, 2007). For large $L$ and a fully connected system, $r=\lfloor L / 2\rfloor$ with periodic boundary conditions, the Fisher matrix with respect to $(\mu, \gamma)$ is

$$
I_{\mu, \mu}=\gamma^{2} S, \quad I_{\gamma, \gamma}=(\mu-J)^{2} S, \quad I_{\mu, \gamma}=-(\mu-J) \gamma S,
$$

with

$$
S \simeq \begin{cases}\frac{L}{2}\left|\frac{J}{(\mu-J) \gamma}\right| \frac{1}{(|\mu-J|+|J \gamma|)^{2}} & \text { if }(\mu \neq J, \gamma \neq 0), \\ \frac{L^{2}}{3 J^{2} \gamma^{4}} & \text { if }(\mu=J, \gamma \neq 0), \\ \frac{L^{2} J^{2}}{(\mu-J)^{4}} & \text { if }(\mu \neq J, \gamma=0) .\end{cases}
$$

The lines $(\mu=J, \gamma \neq 0)$ and $(\mu \neq J, \gamma=0)$ reveal secondorder quantum phase transitions, as well as superextensivity of the Fisher matrix in the volume $L$. At the critical line $\gamma=0$, the ground state is unentangled not only in the eigenmodes but also in the original modes $\left\{c_{j}, c_{j}^{\dagger}\right\}_{j}$.

The fully connected translationally invariant Hamiltonian with open boundary conditions reads

$$
A_{j, l}=(\mu-J) \delta_{j, l}+J, \quad B_{j, l}=J \gamma \operatorname{sgn}(l-j),
$$

with second-order quantum phase transitions at lines $\mu=J$ and $\gamma=0$, and a superextensive Fisher matrix as for periodic boundary conditions with different prefactors (Cozzini, Giorda, and Zanardi, 2007; Zanardi, Cozzini, and Giorda, 2007). For instance, at $(\mu>J, \gamma=0)$

$$
I_{\mu, \mu}=0, \quad I_{\gamma, \gamma}=\frac{L^{2} J^{2}}{3(\mu-J)^{2}}, \quad I_{\mu, \gamma}=0
$$

Another interesting case is the Hamiltonian with nearest neighbor tunneling in the modes $\left\{c_{j}, c_{j}^{\dagger}\right\}_{j}$, periodic boundary conditions, and $J>0$ :

$$
\begin{aligned}
A_{j, l} & =(J-\mu) \delta_{j, l}-J \theta(1-|j-l|), \\
B_{j, l} & =J \gamma \operatorname{sgn}(l-j) \theta(1-|j-l|) .
\end{aligned}
$$

Such a Hamiltonian (Zanardi, Paris, and Campos Venuti, 2008) is also equal to

$$
H_{\text {quasispin }}=\sum_{n=1}^{\lfloor L / 2\rfloor}\left(-\epsilon_{n} \sigma_{n}^{z}+\Delta_{n} \sigma_{n}^{y}\right),
$$

with $\quad \epsilon_{n}=-J \cos (2 \pi n / L)-\mu / 2, \quad \Delta_{n}=-J \gamma \sin (2 \pi n / L)$, and $\left\{\sigma_{n}^{y, z}\right\}_{n}$ are Pauli matrices on $n$ orthogonal $\mathbb{C}^{2}$ subspaces. Equation (103) provides an alternative representation of the Hamiltonian in terms of noninteracting quasispins, and its eigenstates are separable with respect to both the eigenmodes and the quasispins. The same quantum Fisher information scaling holds in both representations as theoretical independent models.

The quantum Fisher information is linear in $L$ away from the critical points, but superextensive at criticality in the leading order for large $L$ (Zanardi and Paunković, 2006; Zanardi, Paris, and Campos Venuti, 2008):

$$
\begin{gathered}
I_{J, J}(|\mu|=2 J, \gamma)=\mathcal{O}\left(\frac{L^{2}}{J^{2} \gamma^{2}}\right), \\
I_{\gamma, \gamma}(|\mu| \leq 2 J, \gamma=0)=\mathcal{O}\left(L^{2}\right) .
\end{gathered}
$$

The origin of the superextensivity of $I_{J, J}$ stems from the fact that the symmetric logarithmic derivative, and thus the optimal estimation of $J$, is close to a single-particle operator in the fermion representation away from the critical point, but is a genuine multiparticle operator close to the critical point. In the canonical ensemble, at $|\mu / 2-J| \lesssim 1 / \beta$, the quantum Fisher information has a divergence around zero temperature $I_{J, J}=\mathcal{O}(L \beta /|J \gamma|)$. The superextensivity of $I_{J, J}$, together with the divergence of the derivative of the geometric phase, is also a universal feature depending only on the slope for the closing of the energy gap between one and zero fermion occupations of certain eigenmodes (Cheng et al., 2017).

Superextensive quantum Fisher information is observed also for tight-binding electrons on the triangular lattice with magnetic flux $\phi / 2$ within each triangle, hopping constants $t_{a}$ $\left(t_{b}\right)$ at edge along the $x(y)$ direction and $t_{c}$ at the third edge (Gong and Tong, 2008). Assuming zero momentum in the $y$ direction (Ino and Kohmoto, 2006), the Hamiltonian can be transformed into Eq. (96), with $B_{j, l}=0$ and

$$
\begin{aligned}
A_{j, l}= & -2 t_{b} \cos (2 \pi \phi j) \delta_{l, j}-\left(t_{a}+t_{c} e^{-2 \pi i \phi(j-1 / 2)}\right) \delta_{l, j-1} \\
& -\left(t_{a}+t_{c} e^{2 \pi i \phi(j+1 / 2)}\right) \delta_{l, j+1},
\end{aligned}
$$

with $j$ and $l$ labeling the sites in the $x$ direction. Consider $L=F_{m}$, the $m$ th Fibonacci number, and

$$
\phi=\frac{F_{m-1}}{F_{m}} \underset{m \rightarrow \infty}{\longrightarrow} \frac{\sqrt{5}-1}{2},
$$

the inverse of the golden ratio in the thermodynamic limit. At the critical line $t_{c}=t_{a}$, numerical computations result in $I_{t_{c} / t_{a}}=\mathcal{O}\left(L^{4.9371}\right)$ if $m=3 L+1$, otherwise $I_{t_{c} / t_{a}}=\mathcal{O}\left(L^{2.0}\right)$. $I_{t_{b} / t_{a}}$ has the same size dependence at the critical line $2 t_{b}=t_{a}$. A more complicated quasifree fermion Hamiltonian describes 
a superconductor with a magnetic impurity (Paunković et al., 2008). A sudden increase of the Bures distance between two reduced ground states of a few modes around the impurity at very close exchange interaction with the impurity was numerically observed, but it is unclear whether the quantum Fisher information with respect to exchange interaction is superextensive.

\section{Hubbard models}

Another class of fermionic systems is described by $L$-mode Hubbard Hamiltonians

$$
\begin{aligned}
H_{\mathrm{HM}}= & -\sum_{\substack{j=1, \ldots, L \\
\sigma=\uparrow, \downarrow}} t_{\sigma}\left(c_{j, \sigma}^{\dagger} c_{j+1, \sigma}+\text { H.c. }\right) \\
& +U \sum_{j=1}^{L} n_{j, \uparrow} n_{j, \downarrow}-\mu \sum_{j, \sigma} n_{j, \sigma},
\end{aligned}
$$

with $n_{j, \sigma}=c_{j, \sigma}^{\dagger} c_{j, \sigma}, t_{\sigma}$ the hopping constants, $U$ the interaction strength, and $\mu$ the external potential. When $t_{\uparrow}=t_{\downarrow}$, the system undergoes a BKT quantum phase transition at $U=0$ and half filling $n=(1 / L) \sum_{j, \sigma}\left\langle n_{j, \sigma}\right\rangle=1 . I_{U}$ is extensive, but $I_{U} / L$ diverges as $1 / n$ for $n \rightarrow 0$ and $U=0$, and as $1 / U^{4}$ around the BKT critical point only if the system size is much larger than the correlation length (Campos Venuti et al., 2008). At zero interaction Eq. (107) is a free fermion Hamiltonian, and thus the ground state is not entangled in the eigenmodes.

In the large $U$ limit and at $n=2 / 3$, a quantum phase transition occurs with control parameter $t_{\downarrow} / t_{\uparrow}$. The quantum Fisher information $I_{t_{\downarrow} / t_{\uparrow}}$ at critical points is superextensive $\mathcal{O}\left(L^{\alpha}\right)$, where the exponent was numerically computed $\alpha \simeq 5.3$ (Gu et al., 2008). In the large $U$ limit, the eigenstates of the Hamiltonian are perturbations of those of the interaction term that are Fock states, thus with vanishingly small entanglement with respect to the modes $\left\{c_{j, \sigma}, c_{j, \sigma}^{\dagger}\right\}_{j, \sigma}$.

\section{Spin-1/2 systems}

The next class of models consists of systems of $N$ spins $1 / 2$, which provide alternative representations of quasifree models. Consider the following complete Hamiltonian, to be specialized later on:

$$
\begin{aligned}
H_{\text {spin }}= & -J \sum_{j=1}^{N-1}\left(\frac{1+\gamma}{2} \sigma_{j}^{x} \sigma_{j+1}^{x}+\frac{1-\gamma}{2} \sigma_{j}^{y} \sigma_{j+1}^{y}+\Delta \sigma_{j}^{z} \sigma_{j+1}^{z}\right) \\
& +d \sum_{j=1}^{N-1}\left(\boldsymbol{\sigma}_{j} \wedge \boldsymbol{\sigma}_{j+1}\right)^{z}-\sum_{j=1}^{N}(h-g j) \sigma_{j}^{z}
\end{aligned}
$$

with anisotropies $\gamma$ and $\Delta$, Dzyaloshinskii-Moriya coupling $d$, magnetic field with uniform value $h$ and gradient $g$, and $\boldsymbol{\sigma}_{j}=\left(\sigma_{j}^{x}, \sigma_{j}^{y}, \sigma_{j}^{z}\right)$.

For the moment, focus on the $X Y$ model with transverse field, i.e., $\Delta=d=g=0$, with $J>0$ and periodic boundary conditions. This Hamiltonian can be transformed into Eq. (96) with (102), $\mu=2 h$, and $L=N$ using the Jordan-Wigner transformation (Giamarchi, 2003). Thus, the $X Y$ Hamiltonian provides an alternative physical setting to implement precision metrology without entanglement in the fermionic eigenmodes.
At zero temperature $I_{h, h}=\left(J^{2} / h^{2}\right) I_{J, J}$, since the ground state depends on $h$ and $J$ only via the ratio $h / J$. The Fisher matrix is extensive with divergent prefactors around critical regions (Zanardi, Giorda, and Cozzini, 2007; Cheng et al., 2017) and is superextensive at criticality as in Eqs. (104) and (105).

In the Ising model, i.e., $\gamma=1$, corrections to the quantum Fisher information around the critical points $|h|=J$ (Chen et al., 2008; Zhou and Barjaktarevič, 2008; Zhou, Zhao, and Li, 2008; You and He, 2015) and at small temperature (Zanardi, Campos Venuti, and Giorda, 2007; Invernizzi et al., 2008) are also linear in $N$ with divergent prefactors. $I_{h, h}$ was also explicitly computed by Damski (2013), Damski and Rams (2014), and You and He (2015). A good estimator of the parameter $J$ is the value inferred by measurements of the total magnetization at least at small system size (Invernizzi et al., 2008).

The divergence of $I_{h, h} / N$ was numerically observed also in the $X X$ model, i.e., $\gamma=0$, approaching the critical field $|h|=J$, with the reduced state of spin blocks (Sacramento, Paunković, and Vieira, 2011). This implies precision estimations of the magnetic field looking only at a part of the system.

Divergent $I_{h, h} / N$ at critical field $|h|=J$ was numerically computed also when an alternating magnetic field $\sum_{j=1}^{N}(-)^{j+1} \delta \sigma_{j}^{z}$ is added to the $X Y$ Hamiltonian (You and $\mathrm{He}, 2015)$. The corresponding quasifree fermion Hamiltonian has the additional term $\sum_{j=1}^{N}(-)^{j+1} \delta c_{j}^{\dagger} c_{j}$.

The $X Y$ and the Ising models are also interesting because the superextensivity of the Fisher matrix $I=\left[I_{\theta, \theta^{\prime}}\right]_{\theta, \theta^{\prime}=h, \gamma}$ is robust against disorder, i.e., with Hamiltonian parameters being Gaussian random variables (Garnerone, Jacobson et al., 2009). In the presence of disorder, quantum phase transitions are broadened to Griffiths phases (Griffiths, 1969; Fisher, 1992, 1995; Sachdev, 1999). Thus, the exponent of $N$ on average $I_{h, h}$ and $I_{\gamma, \gamma}$ is slightly reduced but the superextensivity is broadened in the parameter range. Superextensivity of $I_{h, h}$ at the critical Ising point $|h|=J$ becomes a broad peak, and the superextensive $I_{\gamma, \gamma}$ at the $\gamma=0$ critical line is split into two symmetric broad peaks around $\gamma=0$, where at $\gamma=0$ a local minimum with extensive $I_{\gamma, \gamma}$ arises. Furthermore, the scaling of $I_{h, h}$ of the $X Y$ model at the critical point $|h|=J$ is preserved when a periodic time-oscillating transverse magnetic field is considered, and the state is the Floquet time evolution of the ground state at $t=0$ up to times that scale linearly with the system size for small time-dependent driving (Lorenzo et al., 2017). These robustness features are suitable for practical implementations of precision measurements.

Another interesting system is the $X X Z$ model, i.e., $\gamma=0$ and $J<0$, whose low-energy spectrum for $|\Delta|<1 / 2$ is equivalent to a quasifree boson Hamiltonian known as Luttinger liquid (Giamarchi, 2003). Thus, superextensive ground state quantum Fisher information implies precision measurements without entanglement in the boson eigenmodes. Given the Luttinger liquid parameter

$$
K=\frac{\pi}{2 \arccos (-2 \Delta / \sqrt{1+2 d / J})},
$$

superextensive quantum Fisher information $I_{d}$ is observed with periodic boundary conditions at $d=h=g=0$, 


$$
I_{d}= \begin{cases}\mathcal{O}\left(\frac{N \ln N}{J^{2}}\right) & \text { if } \Delta=\frac{1+\sqrt{5}}{8}, \\ \mathcal{O}\left(\frac{N^{6-8 K}}{J^{2}}\right) & \text { if } \frac{1+\sqrt{5}}{8}<\Delta<\frac{1}{2}, \\ \mathcal{O}\left(\frac{N}{J \ln N}\right)^{2} & \text { if } \Delta=\frac{1}{2},\end{cases}
$$

while, with open boundary conditions,

$$
I_{d}=\mathcal{O}\left(\frac{J^{2} K N^{2}}{\left(J^{2}+4 d^{2}\right)^{2}}\right)
$$

at $h=g=0$ and

$$
I_{g}=\mathcal{O}\left(\frac{K N^{4}}{J^{2} u^{2}}\right)
$$

with

$$
u=\frac{\pi \sqrt{1-4 \Delta^{2}}}{2 \arccos (2 \Delta)},
$$

at $d=h=g=0$ (Greschner, Kolezhuk, and Vekua, 2013). Here the quantum Fisher information is superextensive mainly because the system is gapless, while the quantum phase transition affects only subleading in $N$, even though superextensive, orders of the quantum Fisher information. $I_{d}$ is also equal to the quantum Fisher information $I_{\phi}$ with respect to a twist phase $\phi$ of spin operators $\sigma_{j}^{+} \sigma_{j+1}^{-} \rightarrow \sigma_{j}^{+} \sigma_{j+1}^{-} e^{i \phi}$ (Thesberg and Sørensen, 2011). Via the Jordan-Wigner transformation, the $\Delta=\gamma=0$ case in Eq. (108) is equivalent to a quasifree fermion Hamiltonian with matrix elements given by Eq. (102) with $\gamma=$ $\mu=0$ and with the Dzyaloshinskii-Moriya and the gradient field terms $-(i d / 2) \sum_{j=1}^{N} c_{j}^{\dagger} c_{j+1}+$ H.c. and $\sum_{j=1}^{N} g j c_{j}^{\dagger} c_{j}$, respectively. $I_{d}$ and $I_{g}$ have the same scaling in a generalization of the latter model with spin-1/2 fermions (Greschner, Kolezhuk, and Vekua, 2013), providing a further example of precision measurements without fermion entanglement.

Another model, mapped to a quasifree fermionic Hamiltonian and exhibiting superextensive quantum Fisher information, is the quantum compass chain with periodic boundary conditions (Motamedifar et al., 2013)

$H_{\mathrm{QCC}}=\sum_{j=1}^{N / 2}\left(\sum_{\alpha=x, y} J_{\alpha} \sigma_{2 j-1}^{\alpha} \sigma_{2 j}^{\alpha}+J_{z} \sigma_{2 j}^{z} \sigma_{2 j+1}^{z}\right)-h \sum_{j=1}^{N} \sigma_{j}^{y}$.

Quantum phase transitions occur at critical fields $h_{1,2}=$ $\sqrt{J_{x}\left(J_{y} \pm J_{z}\right)} / 2$ when these values are real. Numerical computations show that $I_{h}=\mathcal{O}\left(N^{\alpha}\right)$ is superextensive with

$$
\alpha \simeq\left\{\begin{array}{lllll}
1.80 \pm 0.02 & \text { for } \frac{J_{x}}{J_{z}}>0, & \frac{J_{y}}{J_{z}}>1 & \text { and } & h=h_{1}, \\
1.98 \pm 0.02 & \text { for } \frac{J_{x}}{J_{z}}>0, & \frac{J_{y}}{J_{z}}>1 & \text { and } & h=h_{2} \\
1.94 \pm 0.02 & \text { for } \frac{J_{x}}{J_{z}}<0, & \frac{J_{y}}{J_{z}}<1 & \text { and } & h=h_{2} \\
2.02 \pm 0.02 & \text { for } \frac{J_{x}}{J_{z}}>0, & \frac{J_{y}}{J_{z}}<1 & \text { and } & h=h_{1}
\end{array}\right.
$$

\section{Topological quantum phase transitions}

The quantum Fisher information is superextensive also in the topological quantum phase transition which have nonlocal order parameters (Zeng et al., 2015). Mosaic models defined on two-dimensional lattices with trivalent vertices, i.e., each vertex is the border among three polygons, and with threebody interaction were numerically studied. The $N$-particle Hamiltonian is

$$
H_{\text {mosaic }}=-\sum_{\substack{\alpha=x, y, z \\(j, l) \in S(\alpha)}} J_{j, l}^{\alpha} \sigma_{j}^{\alpha} \sigma_{l}^{\alpha}-K \sum_{j, l, k} \sigma_{j}^{x} \sigma_{l}^{y} \sigma_{k}^{z},
$$

where $S(\alpha)$ is the set of edges in the $\alpha \in\{x, y, z\}$ direction. $H_{\text {mosaic }}$ can be mapped onto a free Majorana fermion Hamiltonian, thus without entanglement in fermion eigenmodes. When the edge numbers of the three polygons are $(4,8,8), J_{j, l}^{x, y}=J, J_{j, l}^{z}=J_{z}$, and $K=0$, the quantum Fisher information is $I_{J_{z}}=\mathcal{O}\left(N^{1.07615 \pm 0.00005}\right)$ at the critical point $J_{z}=\sqrt{2} J>0$ (Garnerone, Abasto et al., 2009). When the edge numbers are $(3,12,12), J_{j, l}^{x, y, z}=J$ for edges within triangular elementary subcells (Yao and Kivelson, 2007), $J_{j, l}^{x, y, z}=J^{\prime}$ for other links, and $K=0$, the quantum Fisher information is $I_{J^{\prime}}=\mathcal{O}\left(N^{1.078 \pm 0.005}\right)$ at the critical point $J^{\prime}=$ $\sqrt{3} J>0$ (Garnerone, Abasto et al., 2009). $H_{\text {mosaic }}$ on the honeycomb lattice with $J_{j, l}^{\alpha}=J_{\alpha}$ has topological quantum phase transition at the boundaries $\left|J_{x}\right|=\left|J_{y}\right|+\left|J_{z}\right|$, $\left|J_{y}\right|=\left|J_{z}\right|+\left|J_{x}\right|$, and $\left|J_{z}\right|=\left|J_{x}\right|+\left|J_{y}\right|$ with superextensive quantum Fisher information, e.g., $I_{J_{x}}=\mathcal{O}(N \ln N)$ for $J_{y}=$ $J_{z}=J_{x} / 2$ and $K=0$ (Zhao and Zhou, 2009), and $I_{J_{z}}=$ $\mathcal{O}\left(N^{1.08675 \pm 0.00005}\right)$ for $J_{z}=J / 2, J_{x}=J_{y}=J-J_{z} / 2$ and $K=1 / 15$ (Garnerone, Abasto et al., 2009).

Topological quantum phase transition are particularly relevant because of superextensive quantum Fisher information in gapless phases and not only at critical points. In the honeycomb lattice with $K=0, J_{x}+J_{y}+J_{z}=J$, and $J_{x}=J_{y}$, the quantum Fisher information is $I_{J_{z}}=$ $\mathcal{O}\left(N^{1.2535 \pm 0.00005}\right)$ at the critical point $J_{z}=J / 2$ (Yang et al., 2008), and $I_{J_{z}}=\mathcal{O}(N \ln N)$ in the gapless phase $J_{z}<J / 2$ (Gu and Lin, 2009). Superextensivity in the gapless phase is due to the algebraic decay of the correlation function of the $z$ edge, in contrast to exponential decay in the gapped phase $J_{z}>J / 2$ leading to extensive quantum Fisher information.

\section{Nonequilibrium steady states}

Consider now parameter estimation with probes in nonequilibrium steady states of spin- $1 / 2$ chains with boundary noise (Prosen, 2015; Marzolino and Prosen, 2016a; Zunkovic and Prosen, 2010) described by the Markovian master equation (Breuer and Petruccione, 2002; Benatti and Floreanini, 2005)

$$
\begin{aligned}
\frac{\partial \rho_{t}}{\partial t}= & -i\left[H_{X Y Z}, \rho_{t}\right] \\
& +\lambda \sum_{\substack{\alpha=1,2 \\
J=1, N}}\left(\mathcal{L}_{\alpha, j} \rho_{t} \mathcal{L}_{\alpha, j}^{\dagger}-\frac{1}{2}\left\{\mathcal{L}_{\alpha, j}^{\dagger} \mathcal{L}_{\alpha, j}, \rho_{t}\right\}\right),
\end{aligned}
$$


with the $X Y Z$ Hamiltonian, i.e., Eq. (108) with $d=g=0$, and Lindblad operators $\mathcal{L}_{1(2), j}=\sqrt{(1 \pm \mu) / 2} \sigma_{j}^{ \pm}$.

Superextensive quantum Fisher information of the nonequilibrium steady states $\rho_{\infty}$ is observed at the nonequilibrium phase transition and in phases with long-range correlations. In the $X Y$ model $\Delta=0$, superextensive quantum Fisher information was computed at the critical lines $h=0\left[I_{h, h}=\right.$ $\left.\mathcal{O}\left(N^{6}\right)\right]$ and $\gamma=0$ with $|h|<J\left|1-\gamma^{2}\right|\left[I_{\gamma, \gamma}=\mathcal{O}\left(N^{2}\right)\right]$, at the critical points $|h|=J\left|1-\gamma^{2}\right|\left[\left[I_{\theta, \theta^{\prime}}\right]_{\theta, \theta^{\prime}=h, \gamma}=\mathcal{O}\left(N^{6}\right)\right]$, and in the phase with long-range correlations $|h|<J\left|1-\gamma^{2}\right|$ $\left[\left[I_{\theta, \theta^{\prime}}\right]_{\theta, \theta^{\prime}=h, \gamma}=\mathcal{O}\left(N^{3}\right)\right]$ (Banchi, Giorda, and Zanardi, 2014).

In the $X X Z$ model $\gamma=0$, the quantum Fisher information $I_{\Delta}$ is superextensive in the limit of small $\lambda / J$ and for $|\Delta| \leq$ $1 / 2$ at irrational $(\arccos \Delta) / \pi$ (Marzolino and Prosen, 2014, $2016 \mathrm{~b}, 2017)$. If $(\arccos \Delta) / \pi$ is rational

$$
I_{\Delta}=\frac{\lambda^{2} \mu^{2}}{J^{2}}\left(\tilde{\xi} N^{2}+\xi N\right),
$$

where $\xi$ and $\tilde{\xi}$ are constants in $N$ and for $\lambda / J<1 / \sqrt{N}$, thus the quantum Fisher information is not superextensive. Nevertheless, after inserting the value of $\Delta$ in $(\arccos \Delta) / \pi$ and reducing the fraction to lowest term $(\arccos \Delta) / \pi=q / p$ with coprime integers $q$ and $p$, one realizes that the coefficient $\xi$ is unbounded when the denominator $p$ grows. Therefore, $I_{\Delta}$, as a function of $\Delta$, exhibits a fractal-like structure with a different size scaling at irrational $(\arccos \Delta) / \pi$. Moreover, the limit of $(\arccos \Delta) / \pi$ approaching irrational numbers from rationals and the thermodynamic limit do not commute. If the thermodynamic limit is first performed then

$$
I_{\Delta}=\mathcal{O}\left(\frac{\lambda^{2} \mu^{2}}{J^{2}} N^{\sim 5}\right)
$$

with $\lambda / J<1 / \sqrt{N}$. If the thermodynamic limit is postponed after the limit to irrational $(\arccos \Delta) / \pi$ then the quantum Fisher information is still fitted by a superextensive power law with exponent depending on the value of $\Delta$, e.g.,

$$
I_{\Delta}=\mathcal{O}\left(\frac{\lambda^{2} \mu^{2}}{J^{2}} N^{2.32788 \pm 0.0009}\right)
$$

with $\lambda / J<1 / \sqrt{N}$ and $(\arccos \Delta) / \pi$ being the golden ratio. When $\Delta=1 / 2$,

$$
I_{\Delta}=\mathcal{O}\left(\frac{\lambda^{2} \mu^{2}}{J^{2}} N^{4}\right)
$$

with $\lambda / J<1 / N$ in both cases.

In addition, the quantum Fisher information of the reduced state of a single spin at position $k$ scales superextensively also for arbitrary dissipation strength $\lambda$ but only at the critical points $|\Delta|=1$, and with a power law depending on the position $k$ of the spin (Marzolino and Prosen, 2017): e.g., at $\lambda=1, I_{\Delta}=$ $\mathcal{O}\left(N^{\sim 2}\right)$ for $k=1, k=\lfloor N / 2\rfloor$, or $k=N$, and $I_{\Delta}=\mathcal{O}\left(N^{\sim 4}\right)$ for $k=\lfloor N / 4\rfloor$ or $k=\lfloor 3 N / 4\rfloor$. This proves that the anisotropy at $|\Delta|=1$ can be precisely estimated measuring single spin magnetizations along the $z$ axis, or measuring the magnetizations $\sum_{j \in \mathcal{P}}\left\langle\sigma_{j}^{z}\right\rangle$ for any noncentrosymmetric portion $\mathcal{P}$ of the chain, or $\sum_{j \in \mathcal{P}} f\left(\left\langle\sigma_{j}^{z}\right\rangle\right)$ with even functions $f(\cdot)$ for any set $\mathcal{P}$, even centrosymmetric ones.

For either small $\lambda$ or small $\mu$ the steady states $\rho_{\infty}$ of these models are perturbations of the completely mixed state and thus not entangled (Bengtsson and Życzkowski, 2006). In the $X X$ model $\gamma=\Delta=0$, there is no nearest neighbor spin entanglement for a wide range of parameters (Žnidarić, 2012). Nonequilibrium steady state probes are favorable because the quantum Fisher information is superextensive in a whole phase and not only at exceptional parameters. Moreover, the distinguishability of nonequilibrium steady states via Fisher information, thus detectability of nonequilibrium criticality and metrological performances, are enhanced compared to thermal equilibrium systems: e.g., the BKT quantum phase transition at $\Delta=1 / 2$ in the ground state of the $X X Z$ model does not correspond to superextensive quantum Fisher information (Chen et al., 2008; Sun, Kolezhuk, and Vekua, 2015).

\section{Adaptive measurements}

Since critical points without critical phases are isolated values, they should be known in advance in order to set the system at criticality and the benefit of superextensive quantum Fisher information. A partial solution is an adaptive approach (Mehboudi, Correa, and Sanpera, 2016) that is here generalized to phase transitions under reasonable conditions. The idea is to perform several estimates changing the thermal state or the nonequilibrium steady state, in particular, the critical point, at each step according to previous estimates, in order to approach the phase transition ensuring enhanced sensitivity of the control parameter estimation.

Consider any phase transition with control parameter $\theta$ to be estimated and assume the quantum Fisher information

$$
I_{\theta-\theta_{c}}=\frac{\xi N}{\left|\theta-\theta_{c}\right|^{\alpha}}
$$

close to a critical point $\theta_{c}$ with $\alpha>0$ and a prefactor $\xi$. The notation $I_{\theta-\theta_{c}}$ is used to make clear that the quantum Fisher information depends on the difference $\theta-\theta_{c}$ rather than on $\theta$ alone. Sub-shot-noise sensitivity is shown only at $\theta=\theta_{c}$. Assume also that $\theta$ is initially known within a fixed interval $\theta \in\left[\theta_{\min }, \theta_{\max }\right]$ enclosing the value $\theta_{c}$ which can be controlled by other system parameters. First, set the critical point to $\theta_{c}^{(1)}=\theta_{\max }$, ensuring that $\theta<\theta_{c}^{(1)}$, i.e., one is on a welldefined side of the phase transition. Find a first estimate $\theta_{\mathrm{est}}^{(1)}$ for the parameter $\theta$, with an uncertainty that saturates the quantum Cramér-Rao bound. Use therefore a number of measurements $M$ that ensures a small error compared to the original confidence interval

$$
\sigma\left(\theta_{\mathrm{est}}^{(1)}\right)=\left(\frac{1}{M I_{\theta-\theta_{c}^{(1)}}}\right)^{1 / 2} \ll\left(\theta_{\max }-\theta_{\min }\right),
$$

where once more $\sigma\left(\theta_{\text {est }}^{(1)}\right)=\operatorname{Var}\left(\theta_{\text {est }}^{(1)}\right)^{1 / 2}$ is the standard deviation of the estimate. Then, update the critical parameter 
to $\theta_{c}^{(2)}=\theta_{\text {est }}^{(1)}+\sigma\left(\theta_{\text {est }}^{(1)}\right)$. Since $\sigma\left(\theta_{\text {est }}^{(1)}\right) \ll\left(\theta_{\max }-\theta_{\min }\right)$, the new critical point $\theta_{c}^{(2)}$ is now much closer to the true value of $\theta$ than $\theta_{c}^{(1)}$, assuming that the obtained estimate $\theta_{\mathrm{est}}^{(1)}$ (which is random and only on average agrees for an unbiased estimator with $\theta)$ is indeed within an interval of order $\sigma\left(\theta_{\mathrm{est}}^{(1)}\right)$ of the true $\theta$. Hence, in the next round, the quantum Fisher information should be substantially larger. Perform then again sufficiently many times a POVM that allows saturating the quantum Cramér-Rao bound for a new estimate $\theta_{\mathrm{est}}^{(2)}$,

$$
\sigma^{2}\left(\theta_{\mathrm{est}}^{(2)}\right)=\frac{1}{M I_{\theta-\theta_{c}^{(2)}}} \simeq\left(\frac{\left[\sigma\left(\theta_{\mathrm{est}}^{(1)}\right)\right]^{\alpha}}{\xi M N}\right) \propto(M N)^{-(2+\alpha) / 2}
$$

After $k$ iterations, the sensitivity of the estimate $\theta^{(k)}$ saturating the quantum Cramér-Rao bound is

$$
\begin{aligned}
\sigma^{2}\left(\theta_{\mathrm{est}}^{(k)}\right) & =\frac{1}{M I_{\theta-\theta_{c}^{(k)}}} \propto(M N)^{-\sum_{j=0}^{k-1}\left(\alpha^{j} / 2^{j}\right)} \\
& =(M N)^{\left[1-(\alpha / 2)^{k}\right] /(\alpha / 2-1)}
\end{aligned}
$$

achieving sub-shot noise for $\alpha<2$ with the limiting scaling $\sigma^{2}\left(\theta_{\text {est }}\right) \propto(M N)^{2 /(\alpha-2)}$ for $k \rightarrow \infty$.

Within this adaptive scheme, control of Hamiltonian parameters, estimations at each step, and rematerialization or restabilization of states at each step are assumed to be efficiently implementable. This adaptive measurement was proposed to estimate the critical magnetic field $h=J$ of the $X X$ model at small nonzero temperature when the quantum phase transition is smoothened to a phase crossover (Mehboudi, Correa, and Sanpera, 2016), achieving the limiting scaling $\sigma^{2}\left(\theta_{\text {est }}\right) \propto(M N)^{-4 / 3}$. The optimal estimate is derived from measurements of the magnetization along the $z$ direction $\sum_{j} \sigma_{j}^{z}$, since the magnetic field commutes with the spin interaction restoring the classical picture of Lagrange multipliers and quantities fixed on average. The same estimate and that derived from measurements of the variance of $\sum_{j} \sigma_{j}^{x}$ are nearly optimal for the $X Y$ model. It is remarkable that, when the adaptive measurement is applied to the model (97) approaching the critical lines in Eq. (99) from the noncritical region, the critical scalings are consistently recovered.

\section{OUTLOOK}

Most work on quantum-enhanced measurements has investigated the benefits of using quantum entanglement [see Paris (2009), Giovannetti, Lloyd, and Maccone (2011), Pezzè and Smerzi (2014), Tóth and Apellaniz (2014), and Pezzè et al. (2016) for recent reviews]. Indeed, under certain restrictive assumptions (see the Introduction), entanglement can be shown to be necessary if one wants to improve over classical sensitivity. However, going beyond these restrictive assumptions opens up a host of new possibilities of which we have explored a large number in this review. Given the difficulty of producing and maintaining entangled states of a large number of subsystems, some of these may open up new roads to better sensitivities than classically possible with a comparable number of resources in actual experiments.
We conclude this review by challenging yet another common mind-set in the field (which we could not quite escape in this review either), namely, the hunt for faster scaling of the sensitivity, in particular, the quest for a scaling faster than $1 / \sqrt{N}$ with the number of subsystems $N$, and the goal of reaching Heisenberg-limited scaling $1 / N$ : It should be clear [see also Sec. IV.C and the linear-nonlinear comparison in Napolitano et al. (2011)] that scaling of the sensitivity is not per se a desideratum. Any given instrument or measurement is judged by its sensitivity, not the scaling thereof. When the sensitivity is $\sigma\left(\theta_{\text {est }}\right)=\alpha N^{d}$, it is sometimes argued that the prefactor $\alpha$ is irrelevant, because a more rapid scaling necessarily leads to better sensitivity for sufficiently large $N$. While mathematically impeccable, this argument assumes that the scaling persists to sufficiently large $N$ where the possibly small prefactor can be compensated, an assumption that may not be valid in practice. Typically, at some point the model breaks down, and systematic errors arise that scale with a positive power of $N$ and at some point become comparable to the stochastic error quantified by the quantum Cramér-Rao bound. And finally, some large- $N$ catastrophe destroys the instrument and its measurement capability. Such concerns are of course relevant for real-life experiments, for which material properties have to be taken into account. But they may also determine fundamental bounds to achievable precision for various physical quantities that are ultimately linked to the fabric of spacetime at extremely small length and time scales. Such ideas were advanced early on notably by Wigner, who estimated the ultimate achievable precision of atomic clocks: increasing the energy of the used clock states more and more for improving its precision leads ultimately to the formation of a black hole and hence renders reading off the clock impossible (Wigner, 1957). Similar limitations of this kind exist for measurements of lengths (Amelino-Camelia, 1999; $\mathrm{Ng}$ and Dam, 2000) and have been recently explored in more detail for the speed of light in vacuum (Braun, Schneiter, and Fischer, 2017).

While current technology is still far from probing such extreme conditions, we nevertheless arrive to the conclusion that the importance of scaling is to give the functional form for an extrapolation of the sensitivity, a prediction of how well one could measure if one had a given large $N$. The validity of this extrapolation is limited by the range over which the scaling persists, an additional datum not described by the scaling nor by the prefactor. Moreover, there is the possibility of an optimum $N$, beyond which the sensitivity worsens (Nichols et al., 2016). In such a case, the interesting questions are "is the optimum $N_{\text {opt }}$ achievable given available resources" and "what is the actual sensitivity at this optimum?" At such an optimum the local scaling is flat, i.e., the smallest possible uncertainty of an unbiased estimate of the parameter is independent of $N$. Ironically, our discussion of advantageous scaling leads to the conclusion that the best scaling may be no scaling at all.

The relevance of the actually achievable smallest uncertainty rather than its scaling with the number of resources makes it particularly important that alternatives to the use of massive entanglement be investigated as so far the number of subsystems that could be entangled experimentally has 
remained relatively small. We hope that the present review will stimulate further research in this direction.

\section{ACKNOWLEDGMENTS}

D. B. thanks Julien Fraïsse for useful discussions, a careful reading of the manuscript, and pointing out Popoviciu (1935). G. A. acknowledges fruitful discussions with Kavan Modi. S. P. thanks S. L. Braunstein, Cosmo Lupo and Leonardo Banchi for comments on Sec. II. This work was supported in part by the Deutsche Forschungsgemeinschaft through SFB TRR21 and Grant No. BR 5221/1-1, by the European Research Council (ERC) through the StG GQCOP (Grant Agreement No. 637352) and AQUMET (Grant Agreement No. 280169) and the PoC ERIDIAN (Grant Agreement No. 713682), by the Royal Society through the International Exchanges Programme (Grant No. IE150570), by the Foundational Questions Institute (fqxi.org) through the Physics of the Observer Programme (Grant No. FQXi-RFP-1601), the Spanish MINECO/FEDER, the MINECO projects MAQRO (No. FIS2015-68039-P), XPLICA (No. FIS2014-62181-EXP) and Severo Ochoa Grant No. SEV-2015-0522, Catalan 2014SGR-1295, by the European Union Project QUIC (Grant Agreement No. 641122), by Fundació Privada CELLEX, by the Engineering and Physical Sciences Research Council (EPSRC) through the UK Quantum Communications Hub (EP/M013472/1), by the Grants No. J1-5439 and No. N1-0025 of the Slovenian Research Agency, and by the H2020 CSA Twinning Project No. 692194, "RBI-T-WINNING."

\section{REFERENCES}

Aasi, J., et al., 2013, Nat. Photonics 7, 613.

Acin, A., 2001, Phys. Rev. Lett. 87, 177901.

Acosta, V. M., et al., 2009, Phys. Rev. B 80, 115202.

Adesso, G., 2014, Phys. Rev. A 90, 022321.

Adesso, G., T. R. Bromley, and M. Cianciaruso, 2016, J. Phys. A 49, 473001.

Adesso, G., F. Dell'Anno, S. D. Siena, F. Illuminati, and L. A. M. Souza, 2009, Phys. Rev. A 79, 040305(R).

Ahn, C., A. C. Doherty, and A. J. Landahl, 2002, Phys. Rev. A 65, 042301.

Alipour, S., M. Mehboudi, and A. T. Rezakhani, 2014, Phys. Rev. Lett. 112, 120405.

Amelino-Camelia, G., 1999, Nature (London) 398, 216.

Amico, L., R. Fazio, A. Osterloh, and V. Vedral, 2008, Rev. Mod. Phys. 80, 517.

Araki, H., and H. Moriya, 2003, Commun. Math. Phys. 237, 105.

Argentieri, G., F. Benatti, R. Floreanini, and U. Marzolino, 2011, Int. J. Quantum. Inform. 09, 1745.

Armen, M. A., J. K. Au, J.K. Stockton, A.C. Doherty, and H. Mabuchi, 2002, Phys. Rev. Lett. 89, 133602.

Armijo, J., T. Jacqmin, K. Kheruntsyan, and I. Bouchoule, 2011, Phys. Rev. A 83, 021605.

Asbóth, J. K., J. Calsamiglia, and H. Ritsch, 2005, Phys. Rev. Lett. 94, 173602

Audenaert, K. M. R., J. Calsamiglia, R. Muñoz Tapia, E. Bagan, L. Masanes, A. Acin, and F. Verstraete, 2007, Phys. Rev. Lett. 98, 160501.

Augusiak, R., J. Kołodyński, A. Streltsov, M. N. Bera, A. Acín, and M. Lewenstein, 2016, Phys. Rev. A 94, 012339.
Bakr, W. S., J. I. Gillen, A. Peng, S. Fölling, and M. Greiner, 2009, Nature (London) 462, 74.

Balachandran, A., T. Govindarajan, A. de Queiroz, and A. Reyes-Lega, 2013, Phys. Rev. Lett. 110, 080503.

Baldwin, C. H., A. Kalev, and I. H. Deutsch, 2014, Phys. Rev. A 90, 012110.

Banchi, L., S. L. Braunstein, and S. Pirandola, 2015, Phys. Rev. Lett. 115, 260501.

Banchi, L., P. Giorda, and P. Zanardi, 2014, Phys. Rev. E 89, 022102. Banuls, M.-C., J. Cirac, and M. Wolf, 2007, Phys. Rev. A 76, 022311.

Barnett, S. M., C. Fabre, and A. Maître, 2003, Eur. Phys. J. D 22, 513.

Barnum, H., E. Knill, G. Ortiz, R. Somma, and L. Viola, 2004, Phys. Rev. Lett. 92, 107902.

Barnum, H., G. Ortiz, R. Somma, and L. Viola, 2005, Int. J. Theor. Phys. 44, 2127.

Barzanjeh, S., S. Guha, C. Weedbrook, D. Vitali, J. H. Shapiro, and S. Pirandola, 2015, Phys. Rev. Lett. 114, 080503.

Baumgratz, T., M. Cramer, and M. B. Plenio, 2014, Phys. Rev. Lett. 113, 140401.

Baxter, R. J., 1982, Exactly Solved Models in Statistical Mechanics (Academic, New York).

Beau, M., and V. A. Zagrebnov, 2010, Condens. Matter Phys. 13, 23003.

Beenakker, C. W. J., 2006, "Quantum Computers, Algorithms and Chaos," in Proceedings of the International School of Physics "Enrico Fermi" (Societa Italiana di fisica, Bologna, Italy).

Beige, A., D. Braun, and P. L. Knight, 2000, New J. Phys. 2, 22.

Beige, A., D. Braun, B. Tregenna, and P. L. Knight, 2000, Phys. Rev. Lett. 85, 1762.

Bellomo, B., A. De Pasquale, G. Gualdi, and U. Marzolino, 2009, Phys. Rev. A 80, 052108.

Bellomo, B., A. De Pasquale, G. Gualdi, and U. Marzolino, 2010a, Phys. Rev. A 82, 062104.

Bellomo, B., A. De Pasquale, G. Gualdi, and U. Marzolino, 2010b, J. Phys. A 43, 395303.

Beltrán, J., and A. Luis, 2005, Phys. Rev. A 72, 045801.

Benatti, F., and D. Braun, 2013, Phys. Rev. A 87, 012340.

Benatti, F., and R. Floreanini, 2005, Int. J. Mod. Phys. B 19, 3063.

Benatti, F., and R. Floreanini, 2014, Int. J. Quantum. Inform. 12, 1461002 .

Benatti, F., and R. Floreanini, 2016, J. Phys. A 49, 305303.

Benatti, F., R. Floreanini, and U. Marzolino, 2009, Europhys. Lett. 88, 20011.

Benatti, F., R. Floreanini, and U. Marzolino, 2010a, Ann. Phys. (Amsterdam) 325, 924.

Benatti, F., R. Floreanini, and U. Marzolino, 2010b, Phys. Rev. A 81, 012105.

Benatti, F., R. Floreanini, and U. Marzolino, 2011, J. Phys. B 44, 091001.

Benatti, F., R. Floreanini, and U. Marzolino, 2012a, Ann. Phys. (Amsterdam) 327, 1304.

Benatti, F., R. Floreanini, and U. Marzolino, 2012b, Phys. Rev. A 85, 042329.

Benatti, F., R. Floreanini, and U. Marzolino, 2014, Phys. Rev. A 89, 032326.

Benatti, F., R. Floreanini, and M. Piani, 2003, Phys. Rev. Lett. 91, 070402.

Benatti, F., R. Floreanini, and K. Titimbo, 2014, Open Syst. Inf. Dyn. 21, 1440003.

Benatti, F., F. Franchini, R. Floreanini, and U. Marzolino, 2017, Open Syst. Inf. Dyn. 24, 1740004. 
Benatti, F., A. M. Liguori, and A. Nagy, 2008, J. Math. Phys. (N.Y.) 49, 042103

Bendersky, A., and J. P. Paz, 2013, Phys. Rev. A 87, 012122.

Benedict, R.P., 1984, Fundamentals of temperature, pressure and flow measurements (Wiley, New York).

Bengtsson, I., and K. Życzkowski, 2006, Geometry of Quantum States: An Introduction to Quantum Entanglement (Cambridge University Press, Cambridge, England).

Bennett, C. H., D. P. DiVincenzo, C. A. Fuchs, T. Mor, E. Rains, P. W. Shor, J. A. Smolin, and W. K. Wootters, 1999, Phys. Rev. A 59, 1070.

Berrada, K., 2013, Phys. Rev. A 88, 013817.

Berry, D. W., M. Tsang, M. J. W. Hall, and H. M. Wiseman, 2015, Phys. Rev. X 5, 031018.

Berry, D. W., and H. M. Wiseman, 2000, Phys. Rev. Lett. 85, 5098.

Berry, D. W., and H. M. Wiseman, 2002, Phys. Rev. A 65, 043803.

Berry, D. W., and H. M. Wiseman, 2006, Phys. Rev. A 73, 063824.

Berry, D. W., and H. M. Wiseman, 2013, Phys. Rev. A 87, 019901.

Bimbard, E., N. Jain, A. MacRae, and A. I. Lvovsky, 2010, Nat. Photonics 4, 243.

Birchall, P. M., J. L. O’Brien, J. C. F. Matthews, and H. Cable, 2017, Phys. Rev. A 96, 062109.

Bloch, I., J. Dalibard, and W. Zwerger, 2008, Rev. Mod. Phys. 80, 885.

Boixo, S., A. Datta, M. J. Davis, S. T. Flammia, A. Shaji, and C. M. Caves, 2008, Phys. Rev. Lett. 101, 040403.

Boixo, S., A. Datta, M. J. Davis, A. Shaji, A. B. Tacla, and C. M. Caves, 2009, Phys. Rev. A 80, 032103.

Boixo, S., A. Datta, S. T. Flammia, A. Shaji, E. Bagan, and C. M. Caves, 2008, Phys. Rev. A 77, 012317.

Boixo, S., S. T. Flammia, C. M. Caves, and J. M. Geremia, 2007, Phys. Rev. Lett. 98, 090401.

Boixo, S., and C. Heunen, 2012, Phys. Rev. Lett. 108, 120402.

Boixo, S., and R. D. Somma, 2008, Phys. Rev. A 77, 052320.

Bollinger, J. J., W. M. Itano, D. J. Wineland, and D. J. Heinzen, 1996, Phys. Rev. A 54, R4649.

Boto, A. N., P. Kok, D. S. Abrams, S. L. Braunstein, C. P. Williams, and J. P. Dowling, 2000, Phys. Rev. Lett. 85, 2733.

Bouchoule, I., N. J. van Druten, and C. I. Westbrook, 2011, in Atom Chips, edited by J. Reichel and V. Vuletić (Wiley-VCH Verlag GmbH \& Co. KGaA, Weinheim, Germany).

Bouyer, P., and M. Kasevich, 1997, Phys. Rev. A 56, R1083.

Bradshaw, M., S. M. Assad, J. Y. Haw, S.-H. Tan, P. K. Lam, and M. $\mathrm{Gu}, 2016$, "The overarching framework between Gaussian quantum discord and Gaussian quantum illumination," arXiv:1611 $.10020 \mathrm{v} 1$.

Braginsky, V. B., F. Y. Khalili, and K. S. Thorne, 1995, Quantum Measurement (Cambridge University Press, Cambridge), 1st ed.

Brandão, F. G. S. L., and G. Gour, 2015, Phys. Rev. Lett. 115, 070503.

Brankov, J. G., D. M. Danchev, and N. S. Tonchev, 2000, Theory of Critical Phenomena in Finite-Size Systems (World Scientific, Singapore).

Bratteli, O., and D. Robinson, 1987, Operator Algebras and Quantum Statistical Mechanics (Springer, Heidelberg).

Braun, D., 2002, Phys. Rev. Lett. 89, 277901.

Braun, D., 2005, Phys. Rev. A 72, 062324.

Braun, D., 2010, Eur. Phys. J. D 59, 521.

Braun, D., 2011, Europhys. Lett. 94, 68007.

Braun, D., 2012, Europhys. Lett. 99, 49901.

Braun, D., and J. Martin, 2011, Nat. Commun. 2, 223.

Braun, D., and S. Popescu, 2014, Quantum Measurements and Quantum Metrology 2, 44.
Braun, D., F. Schneiter, and U. R. Fischer, 2017, Classical Quantum Gravity 34, 175009.

Braunstein, S. L., and C. M. Caves, 1994, Phys. Rev. Lett. 72, 3439.

Braunstein, S. L., C. M. Caves, and G. J. Milburn, 1996, Ann. Phys. (N.Y.) 247, 135.

Breuer, H.-P., and F. Petruccione, 2002, The Theory of Open Quantum Systems (Oxford University Press, New York).

Brody, D., and N. Rivier, 1995, Phys. Rev. E 51, 1006.

Brody, D. C., and A. Ritz, 2003, J. Geom. Phys. 47, 207.

Bromley, T. R., I. A. Silva, C. O. Oncebay-Segura, D. O. SoaresPinto, E. R. deAzevedo, T. Tufarelli, and G. Adesso, 2017, Phys. Rev. A 95, 052313.

Budker, D., D. F. Kimball, S. M. Rochester, and V. V. Yashchuk, 2000, Phys. Rev. Lett. 85, 2088.

Cable, H., M. Gu, and K. Modi, 2016, Phys. Rev. A 93, 040304.

Calabrese, P., M. Mintchev, and E. Vicari, 2012, Europhys. Lett. 98, 20003.

Campos Venuti, L., M. Cozzini, P. Buonsante, F. Massel, N. Bray-Ali, and P. Zanardi, 2008, Phys. Rev. B 78, 115410.

Campos Venuti, L., and P. Zanardi, 2007, Phys. Rev. Lett. 99, 095701.

Casimir, H. B. G., 1968, in Fundamental Problems in Statistical Mechanics II, edited by E. G. D. Cohen (American Elsevier Publishing Company, New York), p. 188.

Caves, C. M., 1980, Rev. Mod. Phys. 52, 341.

Caves, C. M., 1981, Phys. Rev. D 23, 1693.

Chapeau-Blondeau, F., 2015, Phys. Rev. A 91, 052310.

Chen, S., L. Wang, Y. Hao, and Y. Wang, 2008, Phys. Rev. A 77, 032111.

Cheng, J.-M., M. Gong, G.-C. Guo, and Z.-W. Zhou, 2017, Phys. Rev. A 95, 062117.

Childs, A., J. Preskill, and J. Renes, 2000, J. Mod. Opt. 47, 155.

Childs, P. R. N., 2001, Practical Temperature Measurement (Butterworth-Heinemann, Stone, MA).

Chin, A. W., S. F. Huelga, and M. B. Plenio, 2012, Phys. Rev. Lett. 109, 233601.

Chua, S. S. Y., 2015, Quantum Enhancement of a $4 \mathrm{~km}$ Laser Interferometer Gravitational-Wave Detector (Springer, New York).

Clark, L. A., A. Stokes, and A. Beige, 2016, Phys. Rev. A 94, 023840 .

Clifton, R., and H. Halvorson, 2001, Stud. Hist. Phil. Mod. Phys. $32,1$.

Continentino, M. A., 2001, Quantum Scaling in Many-Body Systems (World Scientific, Singapore).

Cooper, J. J., D. W. Hallwood, and J. A. Dunningham, 2009, J. Phys. B 42, 105301.

Cooper, J. J., D. W. Hallwood, J. A. Dunningham, and J. Brand, 2012, Phys. Rev. Lett. 108, 130402.

Correa, L. A., M. Mehboudi, G. Adesso, and A. Sanpera, 2015, Phys. Rev. Lett. 114, 220405.

Cozzini, M., P. Giorda, and P. Zanardi, 2007, Phys. Rev. B 75, 014439.

Cronin, A., J. Schmiedmayer, and D. Pritchard, 2009, Rev. Mod. Phys. 81, 1051.

Crooks, G. E., 2007, Phys. Rev. Lett. 99, 100602.

D'Alessandro, D., 2007, Introduction to Quantum Control and Dynamics (Chapman and Hall/CRC, Boca Raton), 1st ed.

Damanet, F., D. Braun, and J. Martin, 2016, Phys. Rev. A 94, 033838

Damski, B., 2013, Phys. Rev. E 87, 052131.

Damski, B., and M. Rams, 2014, J. Phys. A 47, 025303.

Dang, H. B., A. C. Maloof, and M. V. Romalis, 2010, Appl. Phys. Lett. 97, 151110. 
D’Ariano, G., C. Macchiavello, and M. Sacchi, 1998, Phys. Lett. A 248, 103.

D'Ariano, G., and M. Paris, 1997, Phys. Rev. A 55, 2267.

Datta, A., S. T. Flammia, and C. M. Caves, 2005, Phys. Rev. A 72, 042316.

Datta, A., and A. Shaji, 2012, Mod. Phys. Lett. B 26, 1230010.

Davis, E., G. Bentsen, and M. Schleier-Smith, 2016, Phys. Rev. Lett. 116, 053601.

Davis, J. P., H. Choi, J. Pollanen, and W. P. Halperin, 2006, Phys. Rev. Lett. 97, 115301.

Davis, S., and G. Gutiérrez, 2012, Phys. Rev. E 86, 051136.

Degen, C. L., F. Reinhard, and P. Cappellaro, 2016, "Quantum sensing," arXiv:1611.02427.

Deléglise, S., I. Dotsenko, C. Sayrin, J. Bernu, M. Brune, J.-M. Raimond, and S. Haroche, 2008, Nature (London) 455, 510.

Demkowicz-Dobrzański, R., M. Jarzyna, and J. Kołodiński, 2015, Prog. Opt. 60, 345.

Demkowicz-Dobrzański, R., and L. Maccone, 2014, Phys. Rev. Lett. 113, 250801.

De Pasquale, A., P. Facchi, G. Florio, V. Giovannetti, K. Matsuoka, and K. Yuasa, 2015, Phys. Rev. A 92, 042115.

Deutsch, C., F. Ramirez-Martinez, C. Lacroûte, F. Reinhard, T. Schneider, J. N. Fuchs, F. Piéchon, F. Laloë, J. Reichel, and P. Rosenbusch, 2010, Phys. Rev. Lett. 105, 020401.

Diósi, L., G. Forgács, B. Lukács, and H. L. Frisch, 1984, Phys. Rev. A 29, 3343.

Dolan, B. P., 1998, Proc. R. Soc. A 454, 2655.

Dorner, U., R. Demkowicz-Dobrzański, B. J. Smith, J. S. Lundeen, W. Wasilewski, K. Banaszek, and I. A. Walmsley, 2009, Phys. Rev. Lett. 102, 040403.

Douglass, D. H. J., B. M. Khorana, and M. Brij, 1965, Phys. Rev. 138, A35.

Dowling, J., 1998, Phys. Rev. A 57, 4736.

Dowling, J., 2008, Contemp. Phys. 49, 125.

Dowling, M., A. Doherty, and H. Wiseman, 2006, Phys. Rev. A 73, 052323.

Duivenvoorden, K., B. M. Terhal, and D. Weigand, 2017, Phys. Rev. A 95, 012305 .

Dunningham, J., K. Buenett, and S. Barnett, 2002, Phys. Rev. Lett. 89, 150401.

Dür, W., M. Skotiniotis, F. Fröwis, and B. Kraus, 2014, Phys. Rev. Lett. 112, 080801.

Eckert, K., J. Schliemann, D. Bruß, and M. Lewenstein, 2002, Ann. Phys. (Amsterdam) 299, 88.

Emch, G., 1972, Algebraic Methods in Statistical Mechanics and Quantum Field Theory (Wiley, New York).

Escher, B. M., R. L. de Matos Filho, and L. Davidovich, 2011, Nat. Phys. 7, 406.

Esteve, J., C. Gross, A. Weller, S. Giovanazzi, and M. K. Oberthaler, 2008, Nature (London) 455, 1216.

Esteve, J., J.-B. Trebbia, T. Schumm, A. Aspect, C. Westbrook, and I. Bouchoule, 2006, Phys. Rev. Lett. 96, 130403.

Etesse, J., R. Blandino, B. Kanseri, and R. Tualle-Brouri, 2014, New J. Phys. 16, 053001.

Etesse, J., M. Bouillard, B. Kanseri, and R. Tualle-Brouri, 2015, Phys. Rev. Lett. 114, 193602.

Farace, A., A. De Pasquale, L. Rigovacca, and V. Giovannetti, 2014, New J. Phys. 16, 073010.

Farace, A., A. D. Pasquale, G. Adesso, and V. Giovannetti, 2016, New J. Phys. 18, 013049.

Ferraro, A., L. Aolita, D. Cavalcanti, F. Cucchietti, and A. Acín, 2010, Phys. Rev. A 81, 052318.

Feynman, R., 1994, Statistical Mechanics (Benjamin, Reading, MA).
Fisher, D. S., 1992, Phys. Rev. Lett. 69, 534.

Fisher, D. S., 1995, Phys. Rev. B 51, 6411.

Foini, L., A. Gambassi, R. Konik, and L. F. Cugliandolo, 2017, Phys. Rev. E 95, 052116.

Fraïsse, J. M. E., 2017, Ph.D. thesis, University Tübingen.

Fraïsse, J. M. E., and D. Braun, 2015, Ann. Phys. (Berlin) 527, 701.

Fraïsse, J. M. E., and D. Braun, 2016, "Hamiltonian extensions in quantum metrology," arXiv:1610.05974.

Fraïsse, J. M. E., and D. Braun, 2017, Phys. Rev. A 95, 062342.

Friis, N., M. Skotiniotis, I. Fuentes, and W. Dür, 2015, Phys. Rev. A 92, 022106.

Fuchs, C. A., and J. V. de Graaf, 1999, IEEE Trans. Inf. Theory 45, 1216.

Fujiwara, A., 2001, Phys. Rev. A 63, 042304.

Fujiwara, A., 2006, J. Phys. A 39, 12489.

Fujiwara, A., and T. Hashizumé, 2002, Phys. Lett. A 299, 469.

Fujiwara, A., and H. Imai, 2003, J. Phys. A 36, 8093.

Gagatsos, C. N., D. Branford, and A. Datta, 2016, Phys. Rev. A 94, 042342 .

Gaiba, R., and M. G. A. Paris, 2009, Phys. Lett. A 373, 934.

Gao, Y., and H. Lee, 2014, Eur. Phys. J. D 68, 347.

Garnerone, S., D. Abasto, S. Haas, and P. Zanardi, 2009, Phys. Rev. A 79, 032302.

Garnerone, S., N. T. Jacobson, S. Haas, and P. Zanardi, 2009, Phys. Rev. Lett. 102, 057205.

Genoni, M. G., M. G. A. Paris, G. Adesso, H. Nha, P. L. Knight, and M. S. Kim, 2013, Phys. Rev. A 87, 012107.

Gerry, C., and P. Knight, 2005, Introductory Quantum Optics (Cambridge University Press, Cambridge).

Ghirardi, G., L. Marinatto, and T. Weber, 2002, J. Stat. Phys. 108, 49.

Ghirardi, G., L. Marinatto, and T. Weber, 2004, Phys. Rev. A 70, 012109.

Giamarchi, T., 2003, Quantum Physics in One Dimension (Oxford University Press, New York).

Giazotto, F., T. T. Heikkilä, A. Luukanen, A. M. Savin, and J. P. Pekola, 2006, Rev. Mod. Phys. 78, 217.

Gilchrist, A., K. Nemoto, W. J. Munro, T. C. Ralph, S. Glancy, S. L. Braunstein, and G. J. Milburn, 2004, J. Opt. B 6, S828.

Gill, R. D., and B. Y. Levit, 1995, Bernoulli 1, 59.

Gilmore, R., 1985, Phys. Rev. A 31, 3237.

Giorgini, S., L. Pitaevskii, and S. Stringari, 2008, Rev. Mod. Phys. 80, 1215.

Giovannetti, V., S. Lloyd, and L. Maccone, 2004, Science 306, 1330.

Giovannetti, V., S. Lloyd, and L. Maccone, 2006, Phys. Rev. Lett. 96, 010401.

Giovannetti, V., S. Lloyd, and L. Maccone, 2011, Nat. Photonics 5, 222.

Girardeau, M., 1960, J. Math. Phys. (N.Y.) 1, 516.

Girardeau, M., 1965, J. Math. Phys. (N.Y.) 6, 1083.

Girolami, D., A. M. Souza, V. Giovannetti, T. Tufarelli, J. G. Filgueiras, R. S. Sarthour, D. O. Soares-Pinto, I. S. Oliveira, and G. Adesso, 2014, Phys. Rev. Lett. 112, 210401.

Girolami, D., T. Tufarelli, and G. Adesso, 2013, Phys. Rev. Lett. 110, 240402.

Goble, D. F., and L. E. H. Trainor, 1965, Phys. Lett. 18, 122.

Goble, D. F., and L. E. H. Trainor, 1966, Can. J. Phys. 44, 27.

Goble, D. F., and L. E. H. Trainor, 1967, Phys. Rev. 157, 167.

Gong, L., and P. Tong, 2008, Phys. Rev. B 78, 115114.

Görlitz, A., et al., 2001, Phys. Rev. Lett. 87, 130402.

Gottesman, D., 1996, Phys. Rev. A 54, 1862.

Gottesman, D., A. Kitaev, and J. Preskill, 2001, Phys. Rev. A 64, 012310.

Grabowski, J., M. Kuś, and G. Marmo, 2011, J. Phys. A 44, 175302. 
Grabowski, J., M. Kuś, and G. Marmo, 2012, J. Phys. A 45, 105301. Greiner, M., I. Bloch, O. Mandel, T. Hänsch, and T. Esslinger, 2001, Phys. Rev. Lett. 87, 160405.

Greschner, S., A. K. Kolezhuk, and T. Vekua, 2013, Phys. Rev. B 88, 195101.

Griffiths, R. B., 1969, Phys. Rev. Lett. 23, 17.

Gross, C., T. Zibold, E. Nicklas, J. Estève, and M. K. Oberthaler, 2010, Nature (London) 464, 1165.

Gu, S.-J., 2010, Int. J. Mod. Phys. B 24, 4371.

Gu, S.-J., H.-M. Kwok, W.-Q. Ning, and H.-Q. Lin, 2008, Phys. Rev. B 77, 245109.

Gu, S.-J., and H.-Q. Lin, 2009, Europhys. Lett. 87, 10003.

Haag, R., 1992, Local Quantum Physics (Springer, Heidelberg).

Hall, M. J. W., D. W. Berry, M. Zwierz, and H. M. Wiseman, 2012, Phys. Rev. A 85, 041802.

Hall, M. J. W., and H. M. Wiseman, 2012, Phys. Rev. X 2, 041006. Halvorson, H., and R. Clifton, 2000, J. Math. Phys. (N.Y.) 41, 1711. Haroche, S., 2013, Rev. Mod. Phys. 85, 1083.

Haroche, S., and J.-M. Raimond, 2006, Exploring the Quantum: Atoms, Cavities and Photons (Oxford University Press, Oxford).

Helstrom, C. W., 1969, J. Stat. Phys. 1, 231.

Helstrom, C. W., 1976, Quantum Detection and Estimation Theory (Academic Press, New York).

Henderson, L., and V. Vedral, 2001, J. Phys. A 34, 6899.

Higgins, B. L., D. W. Berry, S. D. Bartlett, M. W. Mitchell, H. M. Wiseman, and G. J. Pryde, 2009, New J. Phys. 11, 073023.

Higgins, B. L., D. W. Berry, S. D. Bartlett, H. M. Wiseman, and G. J. Pryde, 2007, Nature (London) 450, 393.

Hines, A., R. McKenzie, and G. Milburn, 2003, Phys. Rev. A 67, 013609.

Hofheinz, M., et al., 2009, Nature (London) 459, 546.

Holevo, A. S., 1982, Probabilistic and Statistical Aspect of Quantum Theory (North-Holland, Amsterdam).

Holland, H., P. Kok, and J. Dowling, 2002, J. Mod. Opt. 49, 2325.

Holland, M. J., and K. Burnett, 1993, Phys. Rev. Lett. 71, 1355.

Horodecki, R., P. Horodecki, M. Horodecki, and K. Horodecki, 2009, Rev. Mod. Phys. 81, 865.

Hosten, O., R. Krishnakumar, N. J. Engelsen, and M. A. Kasevich, 2016, Science 352, 1552.

Hu, H., G. Xianlong, and X.-J. Liu, 2014, Phys. Rev. A 90, 013622.

Huang, K., et al., 2015, Phys. Rev. Lett. 115, 023602.

Huang, Z., C. Macchiavello, and L. Maccone, 2016, Phys. Rev. A 94, 012101 .

Huelga, S. F., C. Macchiavello, T. Pellizzari, A. K. Ekert, M. B. Plenio, and J. I. Cirac, 1997, Phys. Rev. Lett. 79, 3865.

Hyllus, P., W. Laskowski, R. Krischek, C. Schwemmer, W. Wieczorek, H. Weinfurter, L. Pezzè, and A. Smerzi, 2012, Phys. Rev. A 85, 022321.

Inguscio, M., and L. Fallani, 2013, Atomic Physics: Precision Measurements and Ultracold Matter (Oxford University Press, New York).

Inguscio, M., W. Ketterle, and C. Salomon, 2006, Eds., Ultra-cold Fermi Gases (IOS Press, Amsterdam).

Ino, K., and M. Kohmoto, 2006, Phys. Rev. B 73, 205111.

Invernizzi, C., M. Korbman, L. Campos Venuti, and M. G. A. Paris, 2008, Phys. Rev. A 78, 042106.

Invernizzi, C., M. G. A. Paris, and S. Pirandola, 2011, Phys. Rev. A 84, 022334

Jahnke, T., S. Lanéry, and G. Mahler, 2011, Phys. Rev. E 83, 011109.

Janke, W., D. A. Johnston, and R. P. K. C. Malmini, 2002, Phys. Rev. E 66, 056119.

Janke, W., D. A. Johnston, and R. Kenna, 2003, Phys. Rev. E 67, 046106.
Janyszek, H., 1986a, Rep. Math. Phys. 24, 11.

Janyszek, H., 1986b, Rep. Math. Phys. 24, 1.

Janyszek, H., 1990, J. Phys. A 23, 477.

Janyszek, H., and R. Mrugała, 1989, Phys. Rev. A 39, 6515.

Janyszek, H., and R. Mrugała, 1990, J. Phys. A 23, 467.

Jaynes, E. T., 1957a, Phys. Rev. 106, 620.

Jaynes, E. T., 1957b, Phys. Rev. 108, 171

Jevtic, S., D. Newman, T. Rudolph, and T. M. Stace, 2015, Phys. Rev. A 91, 012331.

Jiang, Z., 2014, Phys. Rev. A 89, 032128.

Jones, J. A., S. D. Karlen, J. Fitzsimons, A. Ardavan, S. C. Benjamin, G. A. D. Briggs, and J. J. L. Morton, 2009, Science 324, 1166.

Juffmann, T., B. B. Klopfer, T. L. I. Frankort, P. Haslinger, and M. A. Kasevich, 2016, Nat. Commun. 7, 12858.

Juffmann, T., S. A. Koppell, B. B. Klopfer, C. Ophus, R. Glaeser, and M. A. Kasevich, 2016, "Multi-pass transmission electron microscopy," arXiv:1612.04931.

Kacprowicz, M., R. Demkowicz-Dobrzański, W. Wasilewski, K. Banaszek, and I. A. Walmsley, 2010, Nat. Photonics 4, 357.

Kessler, E., I. Lovchinsky, A. Sushkov, and M. Lukin, 2014, Phys. Rev. Lett. 112, 150802.

Ketterle, W., and N. J. van Druten, 1996, Phys. Rev. A 54, 656.

Keyl, M., T. Matsui, D. Schlingemann, and R. Werner, 2006, Rev. Math. Phys. 18, 935.

Keyl, M., D. Schlingemann, and R. Werner, 2003, Quantum Inf. Comput. 3, 281 [http://www.rintonpress.com/journals/qiconline .html\#v18n34].

Khorana, B. M., and D. H. Douglass, 1965, Phys. Rev. 138, A35.

Killoran, N., M. Cramer, and M. Plenio, 2014, Phys. Rev. Lett. 112, 150501 .

Killoran, N., F. E. S. Steinhoff, and M. B. Plenio, 2016, Phys. Rev. Lett. 116, 080402.

Kitagawa, M., and M. Ueda, 1993, Phys. Rev. A 47, 5138.

Klaers, J., 2014, J. Phys. B 47, 243001.

Knill, E., and R. Laflamme, 1998, Phys. Rev. Lett. 81, 5672.

Knott, P. A., T. J. Proctor, A. J. Hayes, J. F. Ralph, P. Kok, and J. A. Dunningham, 2016, Phys. Rev. A 94, 062312.

Köhl, M., and T. Esslinger, 2006, Europhysics News 37, 18.

Kołodyński, J., and R. Demkowicz-Dobrzański, 2010, Phys. Rev. A 82, 053804.

Kominis, I., T. Kornack, J. Allred, and M. Romalis, 2003, Nature (London) 422, 596.

Korbicz, J., J. Cirac, and M. Lewenstein, 2005, Phys. Rev. Lett. 95, 120502.

Kraus, C., M. Wolf, J. Cirac, and G. Giedke, 2009, Phys. Rev. A 79, 012306

Krueger, D. A., 1968, Phys. Rev. 172, 211.

Laghaout, A., J. S. Neergaard-Nielsen, I. Rigas, C. Kragh, A. Tipsmark, and U. L. Andersen, 2013, Phys. Rev. A 87, 043826.

Langen, T., S. Erne, R. Geiger, B. Rauer, T. Schweigier, W. Kuhnert, W. Rohringer, I. E. Mazets, T. Gasenzer, and J. Schmiedmayer, 2015, Science 348, 207.

Lanyon, B. P., M. Barbieri, M. P. Almeida, and A. G. White, 2008, Phys. Rev. Lett. 101, 200501.

Laurenza, R., C. Lupo, G. Spedalieri, S. L. Braunstein, and S. Pirandola, 2018, Quantum Meas. Quantum Metrol. 5, 1.

Leanhardt, A. E., T. Pasquini, M. Saba, A. Schirotzek, Y. Shin, D. Kielpinski, D. E. Pritchard, and W. Ketterle, 2003, Science 301, 1513.

Lee, S. K., J. Cho, and K. S. Choi, 2015, New J. Phys. 17, 113053. Leggett, A., 2001, Rev. Mod. Phys. 73, 307.

Leggett, A., 2006, Quantum Liquids (Oxford University Press, Oxford). 
Lewenstein, M., A. Sanpera, V. Ahufinger, B. Damski, A. Sen, and U. Sen, 2007, Adv. Phys. 56, 243.

Li, Y., B. Zeng, X. Liu, and G. Long, 2001, Phys. Rev. A 64, 054302. Linnemann, D., H. Strobel, W. Muessel, J. Schulz, R. J. Lewis-Swan, K. V. Kheruntsyan, and M. K. Oberthaler, 2016, Phys. Rev. Lett. 117, 013001.

Liu, J., and H. Yuan, 2016, New J. Phys. 18, 093009.

Lloyd, S., 2008, Science 321, 1463.

Lo Franco, R., and G. Compagno, 2016, Sci. Rep. 6, 20603.

Lopaeva, E. D., I. R. Berchera, I. P. Degiovanni, S. Olivares, G. Brida, and M. Genovese, 2013, Phys. Rev. Lett. 110, 153603.

Lorenzo, S., J. Marino, F. Plastina, G. M. Palma, and T. J. G. Apollaro, 2017, Sci. Rep. 7, 5672.

Luis, A., 2004, Phys. Lett. A 329, 8.

Luis, A., 2007, Phys. Rev. A 76, 035801.

Lund, A. P., H. Jeong, T. C. Ralph, and M. S. Kim, 2004, Phys. Rev. A 70, 020101.

Lupo, C., and S. Pirandola, 2016, Phys. Rev. Lett. 117, 190802.

Lupo, C., S. Pirandola, V. Giovannetti, and S. Mancini, 2013, Phys. Rev. A 87, 062310.

MacCormick, C., S. Bergamini, C. Mansell, H. Cable, and K. Modi, 2016, Phys. Rev. A 93, 023805.

Macieszczak, K., R. Demkowicz-Dobrzański, and M. Fraas, 2014, New J. Phys. 16, 113002.

Mahmud, K. W., E. Tiesinga, and P. R. Johnson, 2014, Phys. Rev. A 90, 041602.

Maldonado-Mundo, D., and A. Luis, 2009, Phys. Rev. A 80, 063811.

Mancino, L., M. Sbroscia, I. Gianani, E. Roccia, and M. Barbieri, 2016, "Quantum Simulation of single-qubit thermometry using linear optics," arXiv:1609.01590.

Mandel, L., and E. Wolf, 1965, Rev. Mod. Phys. 37, 231.

Marian, P., and T. A. Marian, 2012, Phys. Rev. A 86, 022340.

Marvian, I., and R. W. Spekkens, 2016, Phys. Rev. A 94, 052324.

Marzolino, U., 2013, Europhys. Lett. 104, 40004.

Marzolino, U., and D. Braun, 2013, Phys. Rev. A 88, 063609.

Marzolino, U., and D. Braun, 2015, Phys. Rev. A 91, 039902.

Marzolino, U., and T. Prosen, 2014, Phys. Rev. A 90, 062130.

Marzolino, U., and T. Prosen, 2016a, Phys. Rev. A 93, 032306.

Marzolino, U., and T. Prosen, 2016b, Phys. Rev. A 94, 039903.

Marzolino, U., and T. Prosen, 2017, Phys. Rev. B 96, 104402.

Matsuzaki, Y., S. C. Benjamin, and J. Fitzsimons, 2011, Phys. Rev. A 84, 012103.

Mehboudi, M., L. A. Correa, and A. Sanpera, 2016, Phys. Rev. A 94, 042121.

Merkel, S. T., J. M. Gambetta, J. A. Smolin, S. Poletto, A. D. Córcoles, B. R. Johnson, C. A. Ryan, and M. Steffen, 2013, Phys. Rev. A 87, 062119.

Micheli, A., D. Jaksch, J. Cirac, and P. Zoller, 2003, Phys. Rev. A 67, 013607.

Mills, D. L., 1964, Phys. Rev. 134, A306.

Miszczak, J. A., Z. Puchała, P. Horodecki, A. Uhlmann, and K. Życzkowski, 2009, Quantum Inf. Comput. 9, 0103 [http://www .rintonpress.com/journals/qiconline.html\#v18n34].

Mitchell, M. W., 2017, Quantum Science and Technology 2, 044005.

Modi, K., A. Brodutch, H. Cable, T. Paterek, and V. Vedral, 2012, Rev. Mod. Phys. 84, 1655.

Modi, K., H. Cable, M. Williamson, and V. Vedral, 2011, Phys. Rev. X 1, 021022 .

Modi, K., T. Paterek, W. Son, V. Vedral, and M. Williamson, 2010, Phys. Rev. Lett. 104, 080501.

Mohseni, M., A. T. Rezakhani, and D. A. Lidar, 2008, Phys. Rev. A 77, 032322 .
Monras, A., 2013, "Phase space formalism for quantum estimation of Gaussian states," arXiv:1303.3682.

Monras, A., and F. Illuminati, 2011, Phys. Rev. A 83, 012315.

Monras, A., and M. G. A. Paris, 2007, Phys. Rev. Lett. 98, 160401.

Monroe, C., D. M. Meekho, B. E. King, and D. J. Wineland, 1996, Science 272, 1131.

Montina, A., and F. T. Arecchi, 1998, Phys. Rev. A 58, 3472.

Moritz, H., T. Stöferle, M. Köhl, and T. Esslinger, 2003, Phys. Rev. Lett. 91, 250402.

Moriya, H., 2006, J. Phys. A 39, 3753.

Motamedifar, M., S. Mahdavifar, S. F. Shayesteh, and S. Nemati, 2013, Phys. Scr. 88, 015003.

Mrugała, R., 1984, Physica A (Amsterdam) 125, 631.

Muessel, W., H. Strobel, D. Linnemann, D. B. Hume, and M. K. Oberthaler, 2014, Phys. Rev. Lett. 113, 103004.

Müller, T., B. Zimmermann, J. Meineke, J.-P. Brantut, T. Esslinger, and H. Moritz, 2010, Phys. Rev. Lett. 105, 040401.

Mullin, W. J., 1997, J. Low Temp. Phys. 106, 615.

Mullin, W. J., and A. R. Sakhel, 2012, J. Low Temp. Phys. 166, 125.

Nagaoka, H., 1988, in Proceedings of the 1988 IEEE International Symposium on Information Theory (IEEE, New York), Vol. 98, p. 577.

Nair, R., 2011, Phys. Rev. A 84, 032312.

Nair, R.,, and Tsang, T., 2016, Phys. Rev. Lett. 117, 190801.

Napolitano, M., M. Koschorreck, B. Dubost, N. Behbood, R. J. Sewell, and M. W. Mitchell, 2011, Nature (London) 471, 486.

Napolitano, M., and M. W. Mitchell, 2010, New J. Phys. 12, 093016. Narnhofer, H., 2003, Phys. Lett. A 310, 423.

Neergaard-Nielsen, J. S., B. M. Nielsen, C. Hettich, K. Mølmer, and E. S. Polzik, 2006, Phys. Rev. Lett. 97, 083604.

Ng, Y. J., and H. v. Dam, 2000, Found. Phys. 30, 795.

Nichols, R., T. R. Bromley, L. A. Correa, and G. Adesso, 2016, Phys. Rev. A 94, 042101.

Nichols, R., P. Liuzzo-Scorpo, P. A. Knott, and G. Adesso, 2017, arXiv: 1711.09132.

Nulton, J. D., and P. Salamon, 1985, Phys. Rev. A 31, 2520.

Okamoto, R., M. Iefuji, S. Oyama, K. Yamagata, H. Imai, A.

Fujiwara, and S. Takeuchi, 2012, Phys. Rev. Lett. 109, 130404.

Ollivier, H., and W. H. Zurek, 2001, Phys. Rev. Lett. 88, 017901.

Osborne, M. F. M., 1949, Phys. Rev. 76, 396.

Oszmaniec, M., R. Augusiak, C. Gogolin, J. Kołodyński, A. Acín, and M. Lewenstein, 2016, Phys. Rev. X 6, 041044.

Ourjoumtsev, A., R. Tualle-Brouri, J. Laurat, and P. Grangier, 2006, Science 312, 83.

Pang, S., and T. A. Brun, 2014, Phys. Rev. A 90, 022117.

Pang, S., and T. A. Brun, 2016, Phys. Rev. A 93, 059901.

Paraoanu, G., and H. Scutaru, 2000, Phys. Rev. A 61, 022306.

Paris, M. G. A., 2009, Int. J. Quantum. Inform. 07, 125.

Paskauskas, R., and L. You, 2001, Phys. Rev. A 64, 042310.

Paunković, N., P. D. Sacramento, P. Nogueira, V. R. Vieira, and V. K. Dugaev, 2008, Phys. Rev. A 77, 052302.

Paunković, N., and V. R. Vieira, 2008, Phys. Rev. E 77, 011129.

Peres, A., 1993, Quantum Theory: Concepts and Methods (Kluwer Academic Publishers, Dordrecht).

Pethick, C., and H. Smith, 2004, Bose-Einstein Condensation in Dilute Gases (Cambridge University Press, Cambridge).

Pezzè, L., and A. Smerzi, 2009, Phys. Rev. Lett. 102, 100401.

Pezzè, L., and A. Smerzi, 2014, in "Atom Interferometry," Proceedings of the International School of Physics "Enrico Fermi," Course 188, edited by G. M. Tino and M. A. Kasevich (IOS Press, Amsterdam), p. 691.

Pezzè, L., A. Smerzi, M. K. Oberthaler, R. Schmied, and P. Treutlein, 2016, "Non-classical states of atomic ensembles: fundamentals and applications in quantum metrology," arXiv:1609.01609. 
Pinel, O., J. Fade, D. Braun, P. Jian, N. Treps, and C. Fabre, 2012, Phys. Rev. A 85, 010101.

Pinel, O., P. Jian, N. Treps, C. Fabre, and D. Braun, 2013, Phys. Rev. A 88, 040102.

Pirandola, S., 2011, Phys. Rev. Lett. 106, 090504.

Pirandola, S., 2016, "Capacities of repeater-assisted quantum communications," arXiv:1601.00966.

Pirandola, S., R. Laurenza, C. Ottaviani, and L. Banchi, 2017, Nat. Commun. 8, 15043.

Pirandola, S., and S. Lloyd, 2008, Phys. Rev. A 78, 012331.

Pirandola, S., and C. Lupo, 2017, Phys. Rev. Lett. 118, 100502.

Pirandola, S., C. Lupo, V. Giovannetti, S. Mancini, and S. L. Braunstein, 2011, New J. Phys. 13, 113012.

Pitaevskii, L., and S. Stringari, 2003, Bose-Einstein Condensation (Oxford University Press, New York).

Plastino, A. R., D. Manzano, and J. Dehesa, 2009, Europhys. Lett. 86, 20005.

Plenio, M. B., S. F. Huelga, A. Beige, and P. L. Knight, 1999, Phys. Rev. A 59, 2468.

Pope, D. T., H. M. Wiseman, and N. K. Langford, 2004, Phys. Rev. A 70, 043812.

Popoviciu, T., 1935, Mathematica 9, 129.

Proctor, T. J., P. A. Knott, and J. A. Dunningham, 2018, Phys. Rev. Lett. 120, 080501.

Prokopenko, M., J. T. Lizier, O. Obst, and X. R. Wang, 2011, Phys. Rev. E 84, 041116.

Prosen, T., 2015, J. Phys. A 48, 373001.

Pustelny, S., A. Wojciechowski, M. Gring, M. Kotyrba, J. Zachorowski, and W. Gawlik, 2008, J. Appl. Phys. 103, 063108.

Quan, H. T., and F. M. Cucchietti, 2009, Phys. Rev. E 79, 031101.

Ragy, S., and G. Adesso, 2012, Sci. Rep. 2, 651.

Ragy, S., M. Jarzyna, and R. Demkowicz-Dobrzański, 2016, Phys. Rev. A 94, 052108.

Raitz, C., A. M. Souza, R. Auccaise, R. S. Sarthour, and I. S. Oliveira, 2015, Quantum Inf. Process. 14, 37.

Ralph, T. C., 2002, Phys. Rev. A 65, 042313.

Reeb, D., and M. M. Wolf, 2015, IEEE Trans. Inf. Theory 61, 1458.

Rehr, J. J., 1970, Phys. Rev. B 1, 3160.

Reichl, L.E., 1998, A modern course in statistical physics (Wiley, New York).

Riedel, C. J., 2015, Phys. Rev. A 92, 010101.

Riedel, M. F., P. Böhi, Y. Li, T. W. Hänsch, A. Sinatra, and P. Treutlein, 2010, Nature (London) 464, 1170.

Rigovacca, L., A. Farace, A. De Pasquale, and V. Giovannetti, 2015, Phys. Rev. A 92, 042331.

Rigovacca, L., A. Farace, L. A. M. Souza, A. De Pasquale, V. Giovannetti, and G. Adesso, 2017, Phys. Rev. A 95, 052331.

Rivas, Á., and A. Luis, 2010, Phys. Rev. Lett. 105, 010403.

Rivas, Á., and A. Luis, 2012, New J. Phys. 14, 093052.

Robertson, H. P., 1929, Phys. Rev. 34, 163.

Robillard, J.-F., A. Devos, I. Roch-Jeune, and P. A. Mante, 2008, Phys. Rev. B 78, 064302.

Roga, W., D. Buono, and F. Illuminati, 2015, New J. Phys. 17, 013031.

Roga, W., S. Giampaolo, and F. Illuminati, 2014, J. Phys. A 47, 365301.

Roy, S. M., and S. L. Braunstein, 2008, Phys. Rev. Lett. 100, 220501.

Ruppeiner, G., 1979, Phys. Rev. A 20, 1608.

Ruppeiner, G., 1981, Phys. Rev. A 24, 488.

Ruppeiner, G., 1991, Phys. Rev. A 44, 3583.

Ruppeiner, G., 1995, Rev. Mod. Phys. 67, 605.

Sacchi, M., 2005, Phys. Rev. A 72, 014305.

Sachdev, S., 1999, Quantum Phase Transitions (Cambridge University Press, Cambridge, England).
Sacramento, P. D. and N. Paunković, and V.R. Vieira, 2011, Phys. Rev. A 84, 062318.

Šafránek, D. 2017, Phys. Rev. A 95, 052320.

Šafránek, D., and I. Fuentes, 2016, Phys. Rev. A 94, 062313.

Šafránek, D., A. R. Lee, and I. Fuentes, 2015, New J. Phys. 17, 073016.

Sakurai, J., 1994, Modern Quantum Mechanics (Addison-Wesley, Reading, MA).

Salamon, P., J. Nulton, and E. Ihrig, 1984, J. Chem. Phys. 80, 436.

Sanders, B., and G. Milburn, 1995, Phys. Rev. Lett. 75, 2944.

Sanner, C., E. J. Su, A. Keshet, R. Gommers, Y.-i. Shin, W. Huang, and W. Ketterle, 2010, Phys. Rev. Lett. 105, 040402.

Sattler, K.D., 2011, Handbook of Nanophysics Principles and Methods (CRC Press, Taylor \& Francis Group, Boca Raton).

Schleich, W., 2001, Quantum Optics in Phase Space (Wiley-VCH Verlag Berlin GmbH, Berlin, Germany).

Schliemann, J., J. Cirac, M. Kus, M. Lewenstein, and D. Loss, 2001, Phys. Rev. A 64, 022303.

Schmitt, J., T. Damm, D. Dung, F. Vewinger, J. Klaers, and M. Weitz, 2014, Phys. Rev. Lett. 112, 030401.

Schuch, N., F. Verstraete, and J. Cirac, 2004, Phys. Rev. A 70, 042310.

Scutaru, H., 1998, J. Phys. A 31, 3659.

Segovia, P., D. Purdie, M. Hengsberger, and Y. Baer, 1999, Nature (London) 402, 504.

Sekatski, P., M. Skotiniotis, J. Kołodyński, and W. Dür, 2017, Quantum Opt. 1, 27.

Serafini, A., 2012, Int. Scholarly Res. Not. 2012, 275016 [https:// www.hindawi.com/journals/isrn/2012/275016/abs/].

Sewell, R. J., M. Koschorreck, M. Napolitano, B. Dubost, N. Behbood, and M.W. Mitchell, 2012, Phys. Rev. Lett. 109, 253605.

Sewell, R. J., M. Napolitano, N. Behbood, G. Colangelo, F. Martin Ciurana, and M. W. Mitchell, 2014, Phys. Rev. X 4, 021045.

Shah, V., G. Vasilakis, and M. V. Romalis, 2010, Phys. Rev. Lett. 104, 013601.

Shi, Y., 2004, J. Phys. A 37, 6807.

Shor, P. W., 1995, Phys. Rev. A 52, R2493.

Söderholm, J., G. Björk, B. Hessmo, and S. Inoue, 2003, Phys. Rev. A 67, 053803.

Sommers, H.-J., and K. Zyczkowski, 2003, J. Phys. A 36, 10083.

Song, H. F., S. Rachel, C. Flindt, I. Klich, N. Laflorencie, and K. Le Hur, 2012, Phys. Rev. B 85, 035409.

Sonin, E. A., 1969, Sov. Phys. JETP 29, 520 [http://www.jetp.ac.ru/ cgi-bin/e/index/e/29/3/p520?a=list].

Sørensen, A., L.-M. Duan, J. Cirac, and P. Zoller, 2001, Nature (London) 409, 63.

Spedalieri, G., C. Lupo, S. L. Braunstein, and S. Pirandola, 2016, "Thermal quantum metrology in memoryless and correlated environments," arXiv:1602.05958.

Spedalieri, G., C. Weedbrook, and S. Pirandola, 2013, J. Phys. A 46, 025304

Stace, T. M., 2010, Phys. Rev. A 82, 011611.

Steane, A. M., 1996, Phys. Rev. Lett. 77, 793.

Streltsov, A., G. Adesso, and M. B. Plenio, 2017, Rev. Mod. Phys. 89, 041003.

Streltsov, A., U. Singh, H. S. Dhar, M. N. Bera, and G. Adesso, 2015, Phys. Rev. Lett. 115, 020403.

Strocchi, F., 1985, Elements of Quantum mechanics of Infinite Systems (World Scientific, Singapore).

Strocchi, F., 2008a, An Introduction to the Mathematical Structure of Quantum Mechanics (World Scientific, Singapore), 2nd ed. 
Strocchi, F., 2008b, Symmetry Breaking (Springer, Heidelberg), 2nd ed.

Strocchi, F., 2012, Eur. Phys. J. Plus 127, 12.

Summers, J., and R. Werner, 1985, Phys. Lett. A 110, 257.

Summers, J., and R. Werner, 1987a, J. Math. Phys. (N.Y.) 28, 2440.

Summers, J., and R. Werner, 1987b, Commun. Math. Phys. 110, 247.

Sun, G., A. K. Kolezhuk, and T. Vekua, 2015, Phys. Rev. B 91, 014418.

Suzuki, S., M. Takeoka, M. Sasaki, U. L. Andersen, and F. Kannari, 2006, Phys. Rev. A 73, 042304.

Tacla, A. B., and C. M. Caves, 2013, New J. Phys. 15, 023008.

Tan, S.-H., B. I. Erkmen, V. Giovannetti, S. Guha, S. Lloyd, L. Maccone, S. Pirandola, and J. H. Shapiro, 2008, Phys. Rev. Lett. 101, 253601.

Tham, W. K., H. Ferretti, A. V. Sadashivan, and A. M. Steinberg, 2016, Sci. Rep. 6, 38822.

The LIGO Scientific Collaboration, 2011, Nat. Phys. 7, 962.

Thesberg, M., and E. S. Sørensen, 2011, Phys. Rev. B 84, 224435.

Thirring, W., 2002, Quantum Mathematical Physics: Atoms, Molecules and Large Systems (Springer, Berlin).

Tichy, M., F. de Melo, M. Kuś, F. Mintert, and A. Buchleitner, 2013, Fortschr. Phys. 61, 225.

Tilma, T., S. Hamaji, W. J. Munro, and K. Nemoto, 2010, Phys. Rev. A 81, 022108.

Tóth, G., 2012, Phys. Rev. A 85, 022322.

Tóth, G., and I. Apellaniz, 2014, J. Phys. A 47, 424006.

Tóth, G., C. Knapp, O. Gühne, and H. Briegel, 2009, Phys. Rev. A 79, 042334

Tsang, M., R. Nair, and X.-M. Lu, 2016, Phys. Rev. X 6, 031033.

Tsuda, Y., and K. Matsumoto, 2005, J. Phys. A 38, 1593.

Tzeng, Y.-C., H.-H. Hung, Y.-C. Chen, and M.-F. Yang, 2008, Phys. Rev. A 77, 062321.

Uys, H., and P. Meystre, 2007, Phys. Rev. A 76, 013804.

van Amerongen, A., 2008, Ann. Phys. (France) 33, 1.

van Amerongen, A., J. van Es, P. Wicke, K. Kheruntsyan, and N. van Druten, 2008, Phys. Rev. Lett. 100, 090402.

van den Berg, M., 1983, J. Stat. Phys. 31, 623.

van den Berg, M., and J. T. Lewis, 1982, Physica A (Amsterdam) 110, 550 .

van den Berg, M., J. T. Lewis, and M. Munn, 1986, Helv. Phys. Acta 59, 1289 [https://www.e-periodica.ch/digbib/view?pid=hpa-001: 1986:59\#1293].

van den Berg, M., J. T. Lewis, and J. V. Pulé, 1986, Helv. Phys. Acta 59, 1271 [https://www.e-periodica.ch/digbib/view?pid=hpa-001: 1986:59\#1275].

van Druten, N. J., and W. Ketterle, 1997, Phys. Rev. Lett. 79, 549.

van Trees, H. L., 2001, Detection, Estimation, and Modulation

Theory (Wiley-Interscience, New York), part i ed.

Vasilakis, G., V. Shah, and M. V. Romalis, 2011, Phys. Rev. Lett. 106, 143601.

Vedral, V., 2003, Central Eur. J. Phys. 2, 289.

Verch, R., and R. Werner, 2005, Rev. Math. Phys. 17, 545.

Vlastakis, B., G. Kirchmair, Z. Leghtas, S. E. Nigg, L. Frunzio, S. M. Girvin, M. Mirrahimi, M. H. Devoret, and R. J. Schoelkopf, 2013, Science 342, 607.

Vogel, W., and J. Sperling, 2014, Phys. Rev. A 89, 052302.

Vogels, J. M., K. Xu, C. Raman, J. R. Abo-Shaeer, and W. Ketterle, 2002, Phys. Rev. Lett. 88, 060402.

Vourdas, A., and J. A. Dunningham, 2005, Phys. Rev. A 71, 013809.

Wakui, K., H. Takahashi, A. Furusawa, and M. Sasaki, 2007, Opt. Express 15, 3568.

Wallis, H., 1996, Quantum Semiclass. Opt. 8, 727.

Wang, X., and B. Sanders, 2003, Phys. Rev. A 68, 012101.
Weedbrook, C., S. Pirandola, R. Garcia-Patron, N. J. Cerf, T. C. Ralph, J. H. Shapiro, and S. Lloyd, 2012, Rev. Mod. Phys. 84, 621.

Weedbrook, C., S. Pirandola, J. Thompson, V. Vedral, and M. Gu, 2016, New J. Phys. 18, 043027.

Weinhold, F., 1975, J. Chem. Phys. 63, 2488.

Weiss, U., 1999, Quantum Dissipative Systems (World Scientific, Singapore), 2nd ed.

Wheatley, T. A., D. W. Berry, H. Yonezawa, D. Nakane, H. Arao, D. T. Pope, T. C. Ralph, H. M. Wiseman, A. Furusawa, and E. H. Huntington, 2010, Phys. Rev. Lett. 104, 093601.

Wigner, E. P., 1957, Rev. Mod. Phys. 29, 255.

Wimberger, S., 2016, Phil. Trans. R. Soc. A 374, 20150153.

Wineland, D. J., 2013, Rev. Mod. Phys. 85, 1103.

Wineland, D. J., J. J. Bollinger, W. M. Itano, and D. J. Heinzen, 1994, Phys. Rev. A 50, 67.

Wiseman, H., and J. Vaccaro, 2003, Phys. Rev. Lett. 91, 097902.

Wiseman, H. M., 1995, Phys. Rev. Lett. 75, 4587.

Wiseman, H. M., S. D. Bartlett, and J. A. Vaccaro, 2004, Laser Spectroscopy p. 307.

Wiseman, H. M., and G. J. Milburn, 2009, Quantum Measurement and Control (Cambridge University Press, Cambridge, England), 1 st ed.

Woolley, M. J., G. J. Milburn, and C. M. Caves, 2008, New J. Phys. 10, 125018.

Yan, Z., P.-W. Huang, and Z. Wang, 2016, Phys. Rev. B 93, 085138.

Yang, S., S.-J. Gu, C.-P. Sun, and H.-Q. Lin, 2008, Phys. Rev. A 78, 012304.

Yao, H., and S. A. Kivelson, 2007, Phys. Rev. Lett. 99, 247203.

Yonezawa, H., et al., 2012, Science 337, 1514.

You, W.-L., and L. He, 2015, J. Phys. Condens. Matter 27, 205601.

You, W.-L., Y.-W. Li, and S.-J. Gu, 2007, Phys. Rev. E 76, 022101.

Yousefjani, R., R. Nichols, S. Salimi, and G. Adesso, 2017, Phys. Rev. A 95, 062307.

Yuan, H., 2016, Phys. Rev. Lett. 117, 160801.

Yuan, H., and C.-H. F. Fung, 2015, Phys. Rev. Lett. 115, 110401.

Yukalov, V., 2009, Laser Phys. 19, 1.

Yukawa, M., K. Miyata, T. Mizuta, H. Yonezawa, P. Marek, R. Filip, and A. Furusawa, 2013, Opt. Express 21, 5529.

Yurke, B., 1986, Phys. Rev. Lett. 56, 1515.

Yurke, B., S. McCall, and J. Klauder, 1986, Phys. Rev. A 33, 4033.

Zanardi, P., L. Campos Venuti, and P. Giorda, 2007, Phys. Rev. A 76, 062318.

Zanardi, P., M. Cozzini, and P. Giorda, 2007, J. Stat. Mech. 07, L02002.

Zanardi, P., P. Giorda, and M. Cozzini, 2007, Phys. Rev. Lett. 99, 100603

Zanardi, P., D. Lidar, and S. Lloyd, 2004, Phys. Rev. Lett. 92, 060402.

Zanardi, P., M. G. A. Paris, and L. Campos Venuti, 2008, Phys. Rev. A 78, 042105.

Zanardi, P., and N. Paunković, 2006, Phys. Rev. E 74, 031123.

Zeng, B., X. Chen, D.-L. Zhou, and X.-G. Wen, 2015, "Quantum Information Meets Quantum Matter-From Quantum Entanglement to Topological Phase in Many-Body Systems," arXiv:1508.02595.

Zhang, J., and M. Sarovar, 2015, Phys. Rev. A 91, 052121.

Zhang, Z., S. Mouradian, F. N. C. Wong, and J. H. Shapiro, 2015, Phys. Rev. Lett. 114, 110506.

Zhang, Z., M. Tengner, T. Zhong, F. N. C. Wong, and J. H. Shapiro, 2013, Phys. Rev. Lett. 111, 010501.

Zhao, J.-H., and H.-Q. Zhou, 2009, Phys. Rev. B 80, 014403.

Zhou, H.-Q., and J. P. Barjaktarevič, 2008, J. Phys. A 41, 412001.

Zhou, H.-Q., J. Zhao, and B. Li, 2008, J. Phys. A 41, 492002. 
Žnidarić, M. 2012, Phys. Rev. A 85, 012324.

Zobay, O., and B. M. Garraway, 2004, Phys. Rev. A 69, 023605.

Zunkovic, B., and T. Prosen, 2010, J. Stat. Mech. 08, P08016.

Zurek, W. H., 2001, Nature (London) 412, 712.
Zwierz, M., C. Pérez-Delgado, and P. Kok, 2010, Phys. Rev. Lett. 105, 180402.

Zwierz, M., C. A. Pérez-Delgado, and P. Kok, 2011, Phys. Rev. Lett. 107, 059904. 